\title{
CLASSICAL EXPANSIONS AND THEIR RELATION TO CONJUGATE HARMONIC FUNCTIONS
}

\author{
BY \\ B. MUCKENHOUPT AND E. M. STEIN
}

\section{Introduction.}

a. Background. The purpose of this paper is to study a variety of "classical" expansions in analogy to ordinary Fourier series and integrals. As our starting point we take those expansions which arise in harmonic analysis of functions on Euclidean spaces, spheres, etc., having appropriate rotational invariance. By analogy we then pass to more extensive classes of expansions and consider, among others, the ultraspherical expansions and their continuous analogues, the FourierBessel (i.e., Hankel) transforms.

The common idea which unifies most of the techniques and results obtained here centers about the study of harmonic functions and their conjugates in several variables, and their variants. Ordinary Fourier series and integrals have an intimate connection with analytic functions, and this relation which they enjoy has long provided a fundamental tool in their study. Thus one of our main objectives is the development of an analogous tool for the other expansions in question.

The connection that is used between various expansions and generalized harmonic and conjugate harmonic functions seems to be of basic importance. In its formal aspects this connection has its roots in the classical literature. More recently, however, some of the ideas have been taken up in the theory of axially symmetric potential theory (see Weinstein [24]) and in the study of pseudoanalytic functions of Bers [2], [3], and Vekua [20]. We now describe in more detail the background and statements of our results $\left({ }^{1}\right)$.

We begin by recalling some of the basic properties of the ultraspherical expansions and Fourier-Bessel transforms. Fix $\lambda>0$, and consider the ultraspherical polynomials of degree $n, P_{n}^{\lambda}(t)$, defined by the generating relation:

$$
\left(1-2 t \omega+\omega^{2}\right)^{-\lambda}=\sum_{k=0}^{\infty} \omega^{k} P_{k}^{\lambda}(t) .
$$

Then the set $\left\{P^{\lambda}(\cos \theta)\right\}$ is orthogonal and complete over $(0, \pi)$ with respect to the measure $(\sin \theta)^{2 \lambda} d \theta$. It leads to the expansion $f(\theta) \sim \sum^{\infty} a_{k} P_{k}^{\lambda}(\cos \theta)$ for an

Received by the editors October 2, 1963.

(1) Some of the results obtained in this paper are also described in the note [16]. 
" 'arbitrary" function $f$ on $(0, \pi)$. In the limiting case $\lambda=0$ we recover the usual cosine expansion because $\lim _{\lambda \rightarrow 0} \lambda^{-1} P_{k}^{\lambda}(\cos \theta)=(2 / k) \cos k \theta$. This, of course, leads to the Fourier expansion for the circle, for even functions (a trivial rotational invariance, in this case). When $\lambda=1 / 2$, the $P_{k}^{\lambda}(\cos \theta)$ are the usual Legendre polynomials of $\cos \theta$. As is well known, these functions arise by considering expansions on the sphere (in Euclidean 3-space) invariant under rotations of a fixed axis. The case $\lambda=1$ should also be noted because then $P_{k}^{\lambda}(\cos \theta)=\sin (k+1) \theta / \sin \theta$. In general, when $2 \lambda$ is integral, $2 \lambda=n-2$, the $P_{k}^{\lambda}$ arise in the Fourier analysis of functions of the surface of the sphere in Euclidean $n$-space, $E_{n}$, which are invariant under the rotations leaving a given axis fixed.

The Fourier-Bessel transforms have certain close analogies with the above. Let $J_{\alpha}(t)$ denote the usual Bessel function of order $\alpha$, and set $V_{\alpha}(t)=t^{-\alpha} J_{\alpha}(t)$. Consider the (Hankel) transformation

$$
f(y) \rightarrow \hat{f}(\xi)=\int_{0}^{\infty} V_{\lambda-1 / 2}(\xi y) f(y) y^{2 \lambda} d y .
$$

(Again $\lambda \geqq 0$.) Then, as is well known, this leads to a unitary transformation of the $L^{2}$-space over $(0, \infty)$ with measure $y^{2 \lambda} d y$. The connection with the usual Fourier transform (which occurs when $2 \lambda$ is integral) is as follows. Suppose we consider functions in $E_{n}$ which are radial (i.e., invariant under all rotations about the origin). Then the standard ( $n$-dimensional) Fourier transform of such a function is again radial; and when so restricted this transform becomes the above Hankel transform (with $2 \lambda=n-2$ ).

To relate the above expansions with generalized harmonic and conjugate harmonic functions we shall be guided by the case when $2 \lambda$ is integral. In the trigonometric case $(\lambda=0)$, we can associate to a generalfunction $f(\theta) \sim \sum_{k=0}^{\infty} a_{k} \cos k \theta$ its Poisson integral $u(x, y)=\sum_{k=0}^{\infty} a_{k} r^{k} \cos k \theta,(x, y)=(r \cos \theta, r \sin \theta)$ which is harmonic in the unit disc and takes on the boundary values $f(\theta)$, as $r \rightarrow 1$. From thefunction $u$ we pass to the conjugate harmonic function $v(x, y)=\sum_{k=1}^{\infty} a_{k} r^{k} \sin k \theta$; and the properties of the analytic function $F(z)=u+i v$ are closely related to the original boundary function $f(\theta)$. In the case of $E_{n}$, when $n>2$, there is an analogous notion of conjugacy, which while not as decisive as in the classical case still has some significant properties (see [16] for a general discussion of this point). For this one makes the definition that an $n$-tuple of functions on $E_{n}, u_{1}, u_{2}, \cdots, u_{n}$, are conjugate if they satisfy the equations

$$
\sum_{i=1}^{n} \frac{\partial u_{i}}{\partial x_{i}}=0, \quad \frac{\partial u_{i}}{\partial x_{j}}=\frac{\partial u_{j}}{\partial x_{i}} .
$$

This system implies, in particular, that each $u_{i}$ is harmonic, and, moreover, that the $u_{i}$ are related in a way which generalizes the usual Cauchy-Riemann equations. Now suppose we have chosen a fixed axis, say along the $x_{1}$ direction 
and we consider functions $u_{1}$ which are invariant under rotations leaving this axis fixed,i.e., functions $u_{1}$ which are radial in the variables $x_{2}, x_{3}, \cdots, x_{n}$. If we set $x=x_{1}, y=\left(x_{2}^{2}+x_{3}^{2}+\cdots+x_{n}^{2}\right)^{1 / 2}$, and $U(x, y)=u_{1}\left(x_{1}, \cdots, x_{n}\right)$, then $U$ satisfies the singular "Laplace equation",

$$
\frac{\partial^{2} U}{\partial x^{2}}+\frac{\partial^{2} U}{\partial y^{2}}+\frac{2 \lambda}{y} \frac{\partial U}{\partial y}=0 \quad(2 \lambda=n-2) .
$$

Moreover, the $n-1$ conjugates $u_{2}, u_{3}, \cdots, u_{n}$ are not essentially different, and in fact can be taken so that $u_{k}=\left(-x_{k} / y\right) V(x, y), k=2, \cdots, n$; the relation between $U$ and $V$, resulting from (1.1) becomes

$$
\begin{aligned}
U_{y}+V_{x} & =0, \\
U_{x}-V_{y}-\frac{2 \lambda}{y} V & =0 .
\end{aligned}
$$

Thus if $\Phi$ satisfies (1.2), and is otherwise "arbitrary," we may take $U=\Phi_{x}$, and $V=-\Phi_{y}$ to be typical solutions of (1.3). If we set $u(x, y)=U(x, y)$, and $v(x, y)=y^{2 \lambda} V(x, y)$ we get the more symmetric system

$$
v_{x}=-y^{2 \lambda} u_{y}, \quad v_{y}=y^{2 \lambda} u_{x} .
$$

$u$ of course satisfies (1.2)and $v$ satisfies the same equation with $\lambda$ replaced by $-\lambda$.

We have just described what holds when $2 \lambda$ is integral, but we take this as our model for the case of general $\lambda \geqq 0$. Let us revert to ultraspherical expansions and consider an arbitrary function

$$
f(\theta) \sim \sum_{k=0}^{\infty} a_{k} P_{k}^{\lambda}(\cos \theta)
$$

and its "Poisson integral"

$$
U(x, y)=f(r, \theta)=\sum_{k=0}^{\infty} a_{k} r^{k} P_{k}^{\lambda}(\cos \theta) .
$$

Then $U$ satisfies the equation (1.2) and we are, therefore, led to its conjugate related to $U$ by (1.3). This gives

$$
V(x, y)=f(r, \theta)=2 \lambda \sum_{k=1}^{\infty} \frac{a_{k} r^{k} \sin \theta}{k+2 \lambda} P_{k-1}^{\lambda+1}(\cos \theta)
$$

and leads to the generalized Hilbert transform, $f(\theta) \rightarrow \tilde{f}(\theta)$, where

$$
\tilde{f}(\theta) \sim 2 \lambda \sum_{k=1}^{\infty} \frac{a_{k}}{k+2 \lambda} \sin \theta P_{k-1}^{\lambda+1}(\cos \theta) .
$$

A similar notion of conjugacy is defined for the Hankel transforms in $\$ 16$ (see (16.7) and (16.8)), and for Fourier-Bessel series in $§ 18$. 
b. Summary of Chapter I. We are now in a position to state our results. These are carried out below in detail for the ultraspherical case; their analogies for the Fourier-Bessel case are then sketched in $\$ 16$.

The results of Chapter I deal mainly with the passage to the "Poisson integral", (1.4), its conjugate (1.5), and the properties of $\lambda$-harmonic functions (i.e., solutions of (1.2)). In $\S 2$ we set down various preliminaries, mostly of a formal nature. In $\$ 3$ we prove a maximum principle appropriate to $\lambda$-harmonic functions. The novelty here arises because we deal with regions that contain the $x$-axis (the line of singularity of (1.2)) in their interior. Thus the usual Hopf maximum principle is not directly applicable. However, this is circumvented because the $\lambda$-harmonic functions that arise by (1.4) are even in $y$. (Similarly, the functions (1.5) have a natural extension for $y<0$ as odd functions of $y$.) This evenness (or oddness) is a feature that recurs repeatedly.

$\S 4$ contains the basic estimates for the kernel of the Poisson integral. It turns out that this kernel $P$, defined by

$$
f(r, \theta)=\int_{0}^{\pi} P(r, \theta, \phi) f(\phi)(\sin \phi)^{2 \lambda} d \phi,
$$

behaves when $\theta$ is "near" $\phi$ in a way which is similar to the Poisson kernel in the trigonometric case. The same kind of similarity holds for the conjugate kernel $Q$, and the differentiated Poisson kernel, $P_{r}$ (see $\S \S 7$ and 12). Obtaining these estimates and applying them is a complicated matter. As such this represents one of the main technical difficulties in this paper. The estimates for $P$ are used in $\$ 5$ to relate the boundary behavior of (1.4) to an appropriate "maximal function" of the boundary values. This leads to a description of Poisson integrals (1.4) and an analogue of the Fatou theorem which are strikingly similar to the case of usual harmonic functions. These results are in $\S 6$. The purpose of $\S \S 7$ and 8 is to prove the basic fact that the conjugacy mapping $f \rightarrow \tilde{f}$ is a bounded operator on $L^{p}, 1<p<\infty$.

$\S \S 9$ and 10 deal with the analogue of the $H^{p}$-spaces for ultraspherical expansions and the results center about the boundary behavior of such functions. The critical case $p=1$ leads to an extension of the classical theorem of F. and $M$. Riesz and states that if $\sum a_{k} P_{k}^{\lambda}(\cos \theta)$ and its conjugate,

$$
2 \lambda \sum\left(a_{k} /(k+2 \lambda)\right) \sin \theta P_{k-1}^{\lambda+1}(\cos \theta
$$

both represent finite measures, then both these measures are absolutely continuous. Two tools are used in obtaining the results for $H$-spaces. The first leads to a global majorization, and follows from the fact that $|F|^{p}$ is a sub-solution of (1.2), where $F=U+i V, U$ and $V$ satisfy the "Cauchy-Riemann equations" (1.3) and $p \geqq 2 \lambda /(2 \lambda+1)$. The second tool is the known "similarity principle" for systems like (1.3) (see [2]); but since this principle is applicable 
only in the case of regular coefficients, it can be used only locally (away from the line of singularity).

c. Summary of Chapter II. It is of interest to ask whether the above notions can be used as tools only in the study of ultraspherical and other expansions without reference being made to them in the final statements of results. This will be seen to be the case in the extension of the Littlewood-Paley theory which is given in Chapter II. The main result obtained-and we we discuss only itis an extension of the Marcinkiewicz multiplier theorem of ordinary Fourier series. It arises from the following problem. Consider a transformation $T$ defined by

$$
T f \sim \sum a_{k} \mu_{k} P_{k}^{\lambda}(\cos \theta)
$$

where

$$
f \sim \sum a_{k} P_{k}^{\lambda}(\cos \theta)
$$

We may ask, what are the condition on the multiplier sequence $\left\{\mu_{k}\right\}$ so that $T$ is a continuous transformation of $L^{p}$ to itself, for appropriate $p$ ? An obvious necessary condition is that, (i) the sequence be uniformly bounded, i.e., $\left|\mu_{k}\right| \leqq M$, for some $M$. An additional condition, that of the Marcinkiewicz theorem, which is sufficient in the case of ordinary Fourier series is that, (ii) $\sum_{k=1}^{n} k\left|\mu_{k}-\mu_{k-1}\right|$ $\leqq M n$, for some $M$ and all $n$.

It is to be noted that the sequences for which $\mu_{k}=1$, for $1 \leqq k \leqq m$, and $\mu_{k}=0$, for $k \geqq m$, satisfy these conditions, with a uniform $M$ for all $m$. These particular multiplier sequences correspond to the operators of partial sums, and for these Pollard [14] has proved the required $L^{p}$ boundedness, whenever $(2 \lambda+1) /(\lambda+1)<p<(2 \lambda+1) / \lambda$; incidentally this range of $p$ cannot be extended. In view of this, it might be expected that in general the conditions (i) and (ii) are sufficient for the multiplier transformation $T$ to be bounded on $L^{p}$, for the above range of $p$. This is indeed the case, as Theorem 10 of $\$ 14$ shows.

The proof of this theorem is lengthy and follows preliminary considerations in $\S \S 11,12$, and 13 . We discuss these sections briefly, so as to indicate how the notions of $\lambda$-harmonic functions and their conjugates enter into the proofs. $\S 11$ is devoted in part, to a variant of Pollard's result on partial sums, and this variant and Pollard's theorem have this in common: The formulas for partial sums involve the functions $P_{k}^{\lambda}$; but to study the partial sums effectively these formulas must betransformed so as to involve both the $P_{k}^{\lambda}$ and their conjugates the $P_{k-1}^{\lambda+1}$.

In $\$ 12$ we deal with the " $g$-function" analogue for ultraspherical series. This basic toolinvolves in its very definition the Poisson integral (in the sense of $\lambda$-harmonic functions) of a given boundary function $f$. The result of this section, is that the $L^{p}$-norm of $g$ is comparable with the $L^{p}$-norm of $f$ for $1<p<\infty$, as 
in the case of Fourier series. $\$ 13$ may be viewed as giving a further refinement of this, leading ultimately to the desired results in $\$ 14$.

d. Summary of Chapter III. This chapter deals with several additional results related to the above. In $\S 15$ our main concern is the convolution structure which is naturally associated to ultraspherical expansions. In the case when $2 \lambda$ is integral this convolution structure is, of course, the one induced from the group of rotations in $n+1$ variables. By the aid of this structure we study the $L^{p}$ properties of potential operators ("fractional integration") analogous to those obtained for Fourier series by Hardy and Littlewood. We prove that if

$$
I_{\alpha}(f) \sim \sum_{k=1}^{\infty} k^{-\alpha} a_{k} P_{k}^{\lambda}(\cos \theta)
$$

then the mapping $f \rightarrow I_{\alpha}(f)$ is continuous from $L^{p}$ to $L^{q}$, where $1<p<q<\infty$, $1 / q=1 / p-\alpha /(2 \lambda+1)$. This result, and others like it, are consequences of the general Theorem 13; this theorem holds for any convolution structure, and seems to have some interest in its own right.

In $\$ 16$ we point out how results similar to those of ultraspherical expansions can be derived for Hankel transforms. $\$ 17$ deals with variants of some of the above results, where certain weight factors are introduced in the $L^{p}$-norms. Finally, in $\S 18$, the basic definitions are given for a possible extension of our results to include the case of Fourier-Bessel (or Dini) series. From our point of view this arises by considering functions harmonic in circular cylinders (of $E_{n+1}$ ) which are invariant under rotations about the axis of the cylinder; and then passing by analogy from this case (which corresponds to $2 \lambda$ being integral), to the case of general positive $\lambda$.

e. Further problems. We wish to discuss briefly several further problems suggested by the above.

(i) When $2 \lambda$ is integral the Bessel functions and ultraspherical polynomials can be interpreted as "spherical functions", associated to symmetric spaces; in the case of Bessel functions these spaces are the Euclidean spaces, and in the case of ultraspherical expansions, these are the spheres. It would be of interest to extend the above results to other symmetric spaces; the ones for which such an extension would seem most probable are the symmetric spaces of rank one. Two illustrations should be given in this regard. First, the spherical functions for the complex projective spaces of dimension $n$ have been determined by Élie Cartan. In the notation of Szegö [18, Chapter IV] they are the Jacobi polynomials $P_{k}^{(\alpha, 0)}$, where $\alpha=n-1$. Thus it is strongly suggested that much of what has been done above has an appropriate variant for the more general Jacobi expansions in terms of the $P_{k}^{(\alpha, 0)}$, where now $\alpha$ is any positive number. A similar situation probably exists for the noncompact symmetric spaces of rank one. This is indicated, at least on the formal level, by the known duality which allows one to 
pass from compact to noncompact symmetric spaces; and more particularly by the spherical functions of the Poincaré half-plane, which have been determined explicitly $\left({ }^{2}\right)$.

(ii) As has been stressed repeatedly, the ultraspherical expansions, Hankel transforms, and Fourier-Bessel series may be considered as arising by taking harmonic functions in respectively Euclidean half-spaces, interior of spheres or interior of cylinders, which are properly invariant under rotaticns. However, the expansions associated with a variety of other regions would seem to be amenable to similar treatment. In this connection we should mention the "conical harmonics" associated to regions bounded by circular cones-which regions have an obvious rotational invariance. It is of interest to point out there is an intimate connection between conical harmonics and spherical functions for the Poincaré half-plane( $\left.{ }^{3}\right)$.

\section{Chapter I}

2. Basic formulae. We consider the ultraspherical polynomials of type $\lambda, P_{n}^{\lambda}(t)$, defined by

$$
\sum_{n=0}^{\infty} \omega^{n} P_{n}^{(\lambda)}(t)=\left(1-2 t \omega+\omega^{2}\right)^{-\lambda}
$$

See Szegö [18, p. 82].

For any ultraspherical expansion of the type $f(\theta) \sim \sum a_{n} P_{n}^{\lambda}(\cos \theta)$, we shall associate the "harmonic" function

$$
f(r, \theta)=\sum a_{n} r^{n} P_{n}^{\lambda}(\cos \theta), \quad 0 \leqq r<1,
$$

and we shall also write it in the form $u(x, y)=f(r, \theta)$, where $x=r \cos \theta, y=r \sin \theta$. It can then be verified that $u(x, y)$ satisfies the differential equation

$$
\Delta_{\lambda}(u)=\frac{\partial^{2} u}{\partial x^{2}}+\frac{\partial^{2} u}{\partial y^{2}}+\frac{2 \lambda}{y} \frac{\partial u}{\partial y}=0
$$

for $(x, y)$ in the upper semi-disc; $x^{2}+y^{2}<1$ and $0<y$.

The formal verification of (2.3) follows easily from the differential equation:

$$
f^{\prime \prime}(\theta)+2 \lambda \cot \theta f^{\prime}(\theta)+n(n+2 \lambda) f(\theta)=0
$$

satisfied by $f(\theta)=P_{n}^{\lambda}(\cos \theta)$.

To the series $f(\theta) \sim \sum a_{n} P_{n}^{\lambda}(\cos \theta)$ we shall also associate its "conjugate", series

(2) See the book of S. Helgason, Differential geometry and symmetric spaces, Academic Press, New York, 1962; Chapter X. The determination of the spherical functions of the complex projective spaces, and of the spheres, is in Cartan's paper, Rend. Circ. Mat. Palermo 53 29), (19217-252.

(3) This connection was brought to the authors' attention by L. Ehrenpreis. For information about conical harmonics see $\mathrm{H}$. Bateman, Partial differential equations of mathematical physics, Dover, New York, 194.; Chapter VI. 


$$
f(\theta) \sim 2 \lambda \sum_{n=1}^{\infty} \frac{a_{n}}{n+2 \lambda} \cdot \sin \theta P_{n-1}^{\lambda+1}(\cos \theta)
$$

and its "conjugate harmonic" series

$$
f(r, \theta)=2 \lambda \sum_{n=1}^{\infty} \frac{a_{n}}{n+2 \lambda} r^{n} \sin \theta P_{n-1}^{\lambda+1}(\cos \theta) .
$$

If we now set

$$
v(x, y)=y^{2 \lambda} f(r, \theta)
$$

then $u$ and $v$ satisfy the "Cauchy-Riemann equations":

$$
v_{x}=-y^{2 \lambda} u_{y}, \quad v_{y}=y^{2 \lambda} u_{x} .
$$

$v$ is harmonic in the sense conjugate to $u$, i.e.,

$$
\Delta_{-\lambda}(v)=\frac{\partial^{2} v}{\partial x^{2}}+\frac{\partial^{2} v}{\partial y^{2}}-\frac{2 \lambda}{y} \frac{\partial v}{\partial y}=0 .
$$

We now pass from formal considerations to precise statements. The functions of $\theta$, such as $P_{n}^{\lambda}(\cos \theta)$ and $f(\theta)$ will always be considered on the interval $0 \leqq \theta \leqq \pi$, unless stated to the contrary.

As is known the functions $P_{n}^{\lambda}(\cos \theta)$ are orthogonal with respect to a measure $d m_{\lambda}=d m_{\lambda}(\theta)$. More precisely we have

where

$$
\begin{aligned}
\int_{0}^{\pi} P_{n}^{\lambda}(\cos \theta) P_{m}^{\lambda}(\cos \theta) d m_{\lambda}(\theta) & \\
= & \delta_{n, m} 2^{1-2 \lambda} \pi[\Gamma(\lambda)]^{-2} \frac{\Gamma(n+2 \lambda)}{(n+\lambda) n !}=\delta_{n m} / \gamma_{n},
\end{aligned}
$$

$$
d m_{\lambda}(\theta)=(\sin \theta)^{2 \lambda} d \theta
$$

See Szegö, [18, p. 81].

Thus we shall define the spaces $L^{p}=L^{p}\left(d m_{\lambda}\right)$ as those whose norm $\|f\|_{p}$ is given by $\|f\|_{p}=\left(\int_{0}^{\pi}|f(\theta)|^{p} d m_{\lambda}(\theta)\right)^{1 / p}$ if $1 \leqq p<\infty$, and $\|f\|_{\infty}=\operatorname{ess} \sup |f(\theta)|$. Also $B$ will denote the Borel measures $d \mu$ on $[0, \pi]$ which satisfy:

$$
\|d \mu\|=\int_{0}^{\pi}(\sin \theta)^{2 \lambda}|d \mu(\theta)|<\infty .
$$

If $f(\theta)$ is a given function $(0, \pi)$ integrable with respect to $d m_{\lambda}(\theta)$, we form its ultraspherical expansion

where

$$
f(\theta) \sim \sum_{n=0}^{\infty} a_{n} P_{n}^{\lambda}(\cos \theta),
$$

$$
a_{n}=\gamma_{n} \int_{0}^{\pi} f(\theta) P_{n}^{\lambda}(\cos \theta) d m_{\lambda}(\theta) .
$$


On account of (2.1) it follows easily that

$$
\left|P_{n}^{\lambda}(\cos \theta)\right| \leqq A n^{2 \lambda-1} .
$$

Remembering the definition of $\gamma_{n}$ given in (2.4) it is then clear that the series

$$
f(r, \theta)=\sum_{n=0}^{\infty} a_{n} r^{n} P_{n}^{\lambda}(\cos \theta)
$$

converges absolutely when $0 \leqq r<1$.

The function $f(r, \theta)$ will be referred to as the "Poisson integral" of $f(\theta)$. There is an explicit formula for it which follows from a theorem of Watson [22]. In fact, define the "Poisson kernel" $P(r, \theta, \phi)$ by

$$
P(r, \theta, \phi)=\sum_{0}^{\infty} r^{n} \gamma_{n} P_{n}^{\lambda}(\cos \theta) P_{n}^{\lambda}(\cos \phi)
$$

Then we have

$$
\begin{aligned}
P(r, \theta, \phi) & =\frac{\lambda}{\pi}\left(1-r^{2}\right) \int_{0}^{\pi} \frac{\sin ^{2 \lambda-1} t}{\left[1-2 r(\cos \theta \cos \phi+\sin \theta \sin \dot{\phi} \cos t)+r^{2}\right]^{\lambda+1}} d t \\
& =\frac{\lambda\left(1-r^{2}\right)}{\pi} \int_{0}^{\pi} \frac{\sin ^{2 \lambda-1} t}{\left[(x-\xi)^{2}+(y-\eta)^{2}+2 y \eta(1-\cos t)\right]^{\lambda+1}} d t,
\end{aligned}
$$

where $(x, y)=(r \cos \theta, r \sin \theta),(\xi, \eta)=(\cos \phi, \sin \phi)$. See also Huber [11].

We thus have

$$
f(r, \theta)=\int_{0}^{\pi} P(r, \theta, \phi) f(\phi) d m_{\lambda}(\phi) .
$$

It should be noted that a similar formalism holds for Borel measures $d \mu$ which have finite norm, in place of integrable functions $f$.

If we now write $u(x, y)=f(r, \theta)$, it is apparent from an examination of the Poisson kernel (2.12), that $u(x, y)$ can be naturally defined in the entire unit disc $x^{2}+y^{2}<1$ in such a manner that $u(x, y)$ is even in $y$ and is indefinitely differentiable (in fact real-analytic) there. It is of interest to observe that it follows from this that the function $r^{n} P_{n}^{\lambda}(\cos \theta)$ is real-analytic in $x$ and $y$. (This also follows from the fact that $P_{n}^{\lambda}(t)=(-)^{n} P_{n}^{\lambda}(-t)$.) We can now verify that $u(x, y)$ satisfies the differential equation (2.3) not just when $y>0$, but also when $y<0$. Thus in the study of ultraspherical expansions we are led to study solutions of the singular "Laplace equation"' (2.3), but only those solutions which have a natural continuation past the singular line $y=0$, are even, and real-analytic in the unit disc.

3. Maximum principle. For second-order elliptic operators like those appearing in (2.3) above there is a well-known maximum principle due to E. Hopf [9]. 
However, this principle is applicable only when matters are restricted to the region of regularity of the coefficients. Thus regions containing the singular line $y=0$ would be excluded from consideration. But it is exactly regions of this kind that are needed in what follows below. It is possible to bypass this difficulty by considering only even functions, as is suggested by the facts observed in the preceding section. Our result is as follows.

THEOREM 1. Let $u(x, y)$ be of class $C^{2}$ in an open region $R$. If $R$ contains segments of the line $y=0$, we shall assume that $u(x, y)=u(x,-y)$ near such segments. Assume that $u$ satisfies $\partial^{2} u / \partial x^{2}+\partial^{2} u / \partial y^{2}+(2 \lambda / y)(\partial u / \partial y) \geqq 0$, for $(x, y) \in R, y \neq 0$, and $\lambda$ is a fixed non-negative number. Then $u$ cannot attain a local maximum in $R$, unless $u$ is a constant.

Proof. Suppose, on the contrary, that $\left(x_{0}, y_{0}\right) \in R$ is a local maximum. We can first assert that $y_{0}=0$. For otherwise $u$ would attain a local maximum at a point of regularity of the coefficients. This would contradict Hopf's maximum principle. We therefore can assume that $y_{0}=0$. To treat this case we shall show that since $\partial^{2} u / \partial x^{2}+\partial^{2} u / \partial y^{2}+(2 \lambda / y)(\partial u / \partial y) \geqq 0$, then $u$ satisfies the following property akin to sub-harmonicity: For all $\rho$ sufficiently small

$$
u\left(x_{0}, 0\right) \int_{0}^{\pi} \sin ^{2 \lambda} \theta d \theta \leqq \int_{0}^{\pi} u\left(x_{0}+\rho \cos \theta, \rho \sin \theta\right) \sin ^{2 \lambda} \theta d \theta .
$$

In fact, we have by Green's theorem, for $u, v \in C^{2}$ of a region $D$ with smooth boundary curve $B$

$$
\begin{gathered}
\iint_{D}\left[v \operatorname{div}\left(|y|^{2 \lambda} \operatorname{grad} u\right)-u \operatorname{div}\left(|y|^{2 \lambda} \operatorname{grad} v\right)\right] d x d y \\
=\int_{B}|y|^{2 \lambda}\left(v \frac{\partial u}{\partial n}-u \frac{\partial v}{\partial n}\right) d s .
\end{gathered}
$$

$\left(\partial / \partial n\right.$ denotes the direction of the outward normal.) We set $\Delta_{\lambda}(u)=u_{x x}+u_{y y}$ $+(2 \lambda / y) u_{y}$, and note that if $r^{-2 \lambda}=\left(\left(x-x_{0}\right)^{2}+y^{2}\right)^{-\lambda}$, the $\Delta_{\lambda}\left(r^{-2 \lambda}\right)=0$ (except when $\left.(x, y)=\left(x_{0}, 0\right)\right)$. Take for $D$ the annulus centered at $\left(x_{0}, 0\right)$, whose outer circle has radius $\rho$ and inner circle radius $\varepsilon$; set $v=r^{-2 \lambda}-c$, where $c=\rho^{-2 \lambda}$ (thus $v=0$ on the outer circle). Then (3.2) gives:

$$
-\int_{c_{\rho}}|y|^{2 \lambda} u \frac{\partial v}{\partial n} d s-\int_{c_{\varepsilon}}|y|^{2 \lambda} v \frac{\partial u}{\partial n} d s+\int_{c_{\varepsilon}}|y|^{2 \lambda} u \frac{\partial v}{\partial n} d s \geqq 0 .
$$

Here $C_{\rho}$ and $C_{\varepsilon}$ denote respectively the circles of radius $\rho$ and $\varepsilon$ taken in the positive direction. If we let $\varepsilon \rightarrow 0$ in the above, then the second integral tends to zero, and the resulting inequality is $(3.1)$. Hence if $\left(x_{0}, 0\right)$ is a local maximum of $u$ then by (3.1) there is a local maximum near $\left(x_{0}, 0\right)$ but lying 
strictly in the upper half-plane. Here again we obtain a contradiction with Hopf's maximum principle and the theorem is proved.

4. Estimate for the Poisson kernel. The results dealing with the behavior of Poisson integrals near the boundary discussed below in $\$ 6$ depend in part on the basic estimates for the Poisson kernel given below. Recall the definition and formulae (2.11) and (2.12).

LEMMA 1. Let us write $\delta=1-r, 1 / 2 \leqq r<1$. Then

$$
\begin{aligned}
& \text { (a) } \quad P(r, \theta, \phi) \leqq C \cdot \delta \frac{\sin ^{-\lambda} \theta \sin ^{-\lambda} \phi}{\delta^{2}+(\theta-\phi)^{2}}, \\
& \text { (b) } \quad P(r, \theta, \phi) \leqq C \cdot \frac{\delta}{\left(\delta^{2}+(\theta-\phi)^{2}\right)^{\lambda+1}} .
\end{aligned}
$$

Proof. Write $\Delta=1-2 r \cos (\theta-\phi)+r^{2}, \sigma=r \sin \theta \sin \phi$. By examination in (2.12) it follows that

where

$$
P(r, \theta, \phi)=I_{1}+I_{2},
$$

$$
I_{1} \leqq C\left(1-r^{2}\right) \int_{0}^{\pi / 2} \frac{t^{2 \lambda-1}}{\left[\Delta+\frac{t^{2} \sigma}{4}\right]^{\lambda+1}} d t
$$

and a similar estimate for $I_{2}$ as an integral taken over $\pi / 2 \leqq t \leqq \pi$. If we make the change of variables $u=(\sigma / \Delta)^{1 / 2}$, we get

$$
I_{1} \leqq C\left(1-r^{2}\right) \frac{\sigma^{-\lambda}}{\Delta} \int_{0}^{\pi(\sigma / \Delta)^{1 / 2 / 2}} \frac{u^{2 \lambda-1}}{\left(1+\frac{u^{2}}{4}\right)^{\lambda+1}} d u
$$

Using the fact that

we get

$$
\int_{0}^{\infty} \frac{u^{2 \lambda-1}}{\left(1+\frac{u^{2}}{4}\right)^{\lambda+1}} d u<\infty
$$

$$
I_{1} \leqq C \cdot \delta \frac{(\sin \theta \sin \phi)^{-\lambda}}{\left(1-2 r \cos (\theta-\phi)+r^{2}\right)} \leqq C \cdot \delta \frac{(\sin \theta \sin \phi)^{-\lambda}}{\delta^{2}+(\theta-\phi)^{2}} .
$$

Using the fact that

gives

$$
\int_{0}^{\omega} \frac{u^{2 \lambda-1}}{\left(1+\frac{u^{2}}{4}\right)^{\lambda+1}} d u \leqq C \omega^{2 \lambda}
$$




$$
I_{1} \leqq C \cdot \delta \cdot \sigma^{-\lambda} \cdot \sigma^{\lambda} \Delta^{-\lambda-1} \leqq C \frac{\delta}{\left(\delta^{2}+(\theta-\phi)^{2}\right)^{\lambda+1}} .
$$

(We have used the easily verified estimate

$$
\frac{1}{\Delta} \leqq \frac{C}{\delta^{2}+(\theta-\phi)^{2}}
$$

if $0 \leqq \theta \leqq \pi, 0 \leqq \phi \leqq \pi, \delta=1-r, 1 / 2 \leqq r<1$.)

Similar estimates hold for $I_{2}$. This proves Lemma 1 .

5. A maximal function. Another basic fact we shall use is the analogue of Hardy-Littlewood "max" function, but in the form appropriate for ultraspherical expansions.

For any $f \in L^{1}\left(d m_{\lambda}(\theta)\right)$ we set

We then have

$$
f^{*}(\theta)=\sup _{h \neq 0 ; 0 \leqq \theta+h \leqq \pi} \frac{\int_{\theta}^{\theta+h}|f(\phi)| d m_{\lambda}(\phi)}{\int_{\theta}^{\theta+h} d m_{\lambda}} .
$$

Lemma 2. $f^{*}(\theta)$ is finite for almost every $\theta$. For any $\alpha>0$, let $E_{\alpha}=\{\theta \mid$ $\left.f^{*}(\theta)>\alpha\right\}$. Then $m_{\lambda}\left(E_{\alpha}\right) \leqq(2 / \alpha) \int|f| d m_{\lambda}$. If in addition $f \in L^{p}\left(d m_{\lambda}\right)$, $1<p \leqq \infty$, then $f^{*} \in L^{p}$ and

$$
\left\|f^{*}\right\|_{p} \leqq A_{p}\|f\|_{p} .
$$

Proof. It is possible to imitate the classical argument or to deduce the present lemma from its known special case. We choose the latter approach. Introduce a new variable $x=x(\theta)=\int_{0}^{\theta} d m_{\lambda}(\phi), d x / d \theta=\sin ^{2 \lambda} \theta$. We then have $0 \leqq x \leqq C_{\lambda}$, where $C_{\lambda}=\int_{0} d m_{\lambda}(\phi)$. Let now $F(x)=f(\theta)$, then

$$
m_{\lambda}\left(E_{\alpha}\right)=m_{0}\left\{x\left|k^{-1} \int_{x}^{x+k}\right| F(x) \mid d x>\alpha, 0 \leqq x+k \leqq C_{\lambda}\right\},
$$

where $m_{0}$ denotes the standard Lebesgue measure. We then have by Riesz'slemma

$$
m_{\lambda}\left(E_{\alpha}\right) \leqq \frac{2}{\alpha} \int_{0}^{c_{\lambda}}|F(x)| d x=\frac{2}{\alpha} \int_{0}^{\pi}|f(\phi)| d m_{\lambda}(\phi) .
$$

Similarly the inequality $\left\|f^{*}\right\|_{p} \leqq A_{p}\|f\|_{p}$ is proved.

In view of the lemma we have just proved, the basic lemma for the behavior of Poisson integrals near the boundary can now be stated:

Lemma 3. Let $f \in L^{1}\left(d m_{\lambda}\right)$. If $f(r, \theta)$ denotes the Poisson integral (2.13), and $f^{*}(\theta)$ is the maximal function defined above, then

$$
\sup _{0 \leqq r<1}|f(r, \theta)| \leqq C f^{*}(\theta) \text {. }
$$


Proof. To simplify matters we divide the square $0 \leqq \theta \leqq \pi, 0 \leqq \phi \leqq \pi$, into four parts: (1) $0 \leqq \theta \leqq \pi / 2,0 \leqq \phi \leqq \pi / 2$, (2) $0 \leqq \theta \leqq \pi / 2, \pi / 2 \leqq \phi \leqq \pi$, (3) $\pi / 2 \quad \theta \leqq \pi, 0 \leqq \phi \leqq \pi / 2$, (4) $\pi / 2 \leqq \theta \leqq \pi$, and $\pi / 2 \leqq \phi \leqq \pi$.

Thus we have $f(r, \theta)=f^{1}(r, \theta)+f^{2}(r, \theta)+f^{3}(r, \theta)+f^{4}(r, \theta)$ where say $f^{1}(r, \theta)$ is the Poisson integral of the function which equals $f(\phi)$ for $0 \leqq \phi \leqq \pi / 2$ and vanishes outside this interval; also in $f^{1}(r, \theta)$ we restrict $\theta$ by $0 \leqq \theta \leqq \pi / 2$. Similarly for the other $f^{j}(r, \theta)$. We assume also, without loss of generality that $f \geqq 0$. It turns out that consideration of $f^{1}(r, \theta)$ and $f^{4}(r, \theta)$ are essentially identical $\left({ }^{4}\right)$, while consideration of $f^{2}(r, \theta)$ and $f^{3}(r, \theta)$ are somewhat simpler. We shall therefore discuss in detail the estimates for $f^{1}(r, \theta)$.

Now in the range $0 \leqq \theta \leqq \pi / 2,0 \leqq \phi \leqq \pi / 2, c \theta \leqq \sin \theta \leqq \theta$, and $c \phi \leqq \sin \phi \leqq \phi$, where $c>0$. In the estimates that follow, therefore, we shall systematically replace $\sin \theta$ by $\theta$ and $\sin \phi$ by $\phi$. We now break up matters into further cases:

Case I. $2 \delta=2(1-r) \leqq \theta$.

Let $E$ be the set of $\phi$ 's so that $|\theta-\phi| \leqq \delta, 0 \leqq \phi \leqq \pi / 2$, then

$$
\begin{aligned}
\int_{E} P(r, \theta, \phi) f(\phi) \sin ^{2 \lambda} \phi d \phi & \leqq c \int_{E} \delta^{-1} \frac{f(\phi)(\sin \phi)^{2 \lambda}}{(\sin \theta \sin \phi)^{\lambda}} d \phi \\
& \leqq \frac{c \delta^{-1}}{\theta^{\lambda}} \int_{E} f(\phi) \phi^{\lambda} d \phi
\end{aligned}
$$

by estimate (a) of Lemma 1 for the Poisson kernel. However, $|\phi| \theta \mid \leqq c$ according to the restrictions made on $\theta$ and $\phi$. Thus we have

$$
\int_{E} P(r, \theta, \phi) f(\phi) \sin ^{2 \lambda} \phi d \phi \leqq c \frac{\delta^{-1}}{\theta^{2 \lambda}} \int_{E} f(\phi) \phi^{2 \lambda} d \phi .
$$

However, $\int_{E} \sin ^{2 \lambda} \phi d \phi \geqq c \int_{E} \phi^{2 \lambda} d \phi \geqq c \delta \theta^{2 \lambda} \geqq c \delta(\sin \theta)^{2 \lambda}$ because $|\theta-\phi| \leqq \delta$ and $2 \delta \leqq \theta$. Thus finally:

$$
\int_{E} P(r, \theta, \phi) f(\phi) \sin ^{2 \lambda} \phi d \phi \leqq C \frac{\int_{|\theta-\phi|<\delta} f(\phi) \sin ^{2 \lambda} \phi d \phi}{\int_{|\theta-\phi|<\delta} \sin ^{2 \lambda} \phi d \phi} \leqq C f^{*}(\theta) .
$$

Next let $F$ be the set where $\delta \leqq|\theta-\phi| \leqq \theta / 2$, and $F_{n}$ the set where $2^{n} \delta \leqq|\theta-\phi| \leqq 2^{n+1} \delta$.

Then

$$
\begin{aligned}
\int_{F} P(r, \theta, \phi) f(\phi) \sin ^{2 \lambda} \phi d \phi & \leqq C \delta \int_{F} \frac{f(\phi) \phi^{2 \lambda}}{\theta^{\lambda} \phi^{\lambda}(\theta-\phi)^{2}} d \phi \\
& \leqq c \sum_{n=0}^{\infty} \delta \int_{F_{n}} \frac{f(\phi) \phi^{2 \lambda}}{\theta^{2 \lambda} 2^{2 n} \delta^{2}} d \phi .
\end{aligned}
$$

(4) This is due to the fact that $P(r, \theta, \phi)=P(r, \pi-\theta, \pi-\phi)$. 
This follows by again applying estimate (a) of (4.1) on $P(r, \theta, \phi)$. Now

$$
\int_{F_{n}} \sin ^{2 \lambda} \phi d \phi \geqq c \quad \int_{2^{n} \delta \leqq|\theta-\phi| \leqq 2^{n+1} \delta} \phi^{2 \lambda} d \phi \geqq c \theta^{2 \lambda} \delta 2^{n},
$$

since $\theta \geqq 2 \delta$.

Thus we obtain

$$
\begin{aligned}
\int_{F} P(r, \theta, \phi) f(\phi) \sin ^{2 \lambda} \phi d \phi & \leqq C \sum 2^{-n} \frac{\int_{F_{n}} f(\phi)(\phi)^{2 \lambda} d \phi}{\int_{F_{n}} \sin ^{2 \lambda} \phi d \phi} \\
& \leqq C \sum 2^{-n} f^{*}(\theta)=C f^{*}(\theta)
\end{aligned}
$$

Similarly, let $G$ be the set where $\theta / 2<|\theta-\phi|$, and let $G_{n}$ be the set where $2^{n} \theta<|\theta-\phi| \leqq 2^{n+1} \theta$.

Then

$$
\begin{aligned}
\int_{G} P(r, \theta, \phi) f(\phi) \sin ^{2 \lambda} \phi d \phi & \leqq C \int_{G} \frac{\delta f(\phi) \phi^{2 \lambda}}{(\theta-\phi)^{2 \lambda+2}} d \phi \\
& \leqq C \sum_{n=-1}^{\infty} \theta^{-(2 \lambda+1)} \int_{G_{n}} \frac{f(\phi) \phi^{2 \lambda}}{2^{2 n(\lambda+1)}} d \phi
\end{aligned}
$$

by estimate (b) of ( 4.1$)$, and since $\theta \geqq 2 \delta$.

However,

$$
\int_{G_{n}} \sin ^{2 \lambda} \phi d \phi \geqq c \quad \int_{2^{n} \theta<|\theta-\phi| \leqq 2^{n+1} \theta} \phi^{2 \lambda} d \phi \geqq c\left(2^{n} \theta\right)^{2 \lambda+1} .
$$

Thus the sum is dominated by

$$
c \sum 2^{-n} \frac{\int_{G_{n}} f(\phi) \phi^{2 \lambda} d \phi}{\int_{G_{n}} \sin _{1}^{2 \lambda} \phi d \phi} \leqq c \sum 2^{-n} f^{*}(\theta) .
$$

Combination of the estimates for sets $E, F$, and $G$ shows that

$$
f^{1}(r, \theta) \leqq c f^{*}(\theta) \text { if } 2 \delta \leqq \theta .
$$

The remaining case for $f^{1}(r, \theta)$, that is the case $2 \delta>\theta$, is treated similarly.

Case II. $2 \delta>\theta$.

We sketch the argument:

Let $E$ be the set where $|\theta-\phi|<\delta$. Then

$$
\int_{E} P(r, \theta, \phi) f(\phi) \sin ^{2 \lambda} \phi d \phi \leqq c \delta^{-2 \lambda-1} \int_{E} f(\phi) \phi^{2 \lambda} d \phi \leqq c f^{*}(\theta)
$$


because of estimate (b) of (4.1) and the fact that

$$
\int_{|\theta-\phi|<\delta} \sin ^{2 \lambda} \phi d \phi \geqq c \delta^{2 \lambda+1} \quad \text { if } 2 \delta \geqq \theta .
$$

Finally, let $F$ be the set where $\delta \leqq|\theta-\phi|$, and let $F_{n}$ be the set where $2^{n} \delta \leqq|\theta-\phi|<2^{n+1} \delta$. Then

$$
\begin{aligned}
\int_{F} P(r, \theta, \phi) f(\phi) \sin ^{2 \lambda} \phi d \phi & \leqq c \delta \int_{F} \frac{f(\phi) \phi^{2 \lambda}}{(\theta-\phi)^{2 \lambda+2}} d \phi \\
& \leqq c \sum_{n=0}^{\infty} \int_{F_{n}} \delta^{-2 \lambda-1} \frac{f(\phi) \phi^{2 \lambda} d \phi}{2^{2 n(\lambda+1)}} \leqq c \quad \sum \frac{f^{*}(\theta)}{2^{n}} \leqq c f^{*}(\theta)
\end{aligned}
$$

as in the previous arguments.

This completes the estimate $f^{1}(r, \theta) \leqq c f^{*}(\theta)$ for all $r, \theta$. As mentioned before, $f^{2}, f^{3}$, and $f^{4}$ are treated similarly. This concludes the proof of the lemma.

6. Poisson integrals and the analogue of Fatou's theorem. The following theorem contains the basic results of Poisson integrals for $L^{p}$.

Theorem 2. Let $f \in L^{p}\left(d m_{\lambda}\right), 1 \leqq p \leqq \infty$, and let $f(r, \theta)$ denote its Poisson integral, (2.13). Then

(a) $\|f(r, \theta)\|_{p} \leqq\|f(\theta)\|_{p}, 1 \leqq p \leqq \infty$,

(b) $\|f(r, \theta)-f(\theta)\|_{p} \rightarrow 0$ as $r \rightarrow 1$, if $1 \leqq p<\infty$,

(c) $\lim _{r \rightarrow 1} f(r, \theta)=f(\theta)$ almost everywhere, $1 \leqq p \leqq \infty$,

(d) $\left\|\sup _{r<1}|f(r, \theta)|\right\|_{p} \leqq A_{p}\|f\|_{p}$, if $1<p \leqq \infty$.

Proof. (a) We note that $P(r, \theta, \phi) \geqq 0, P(r, \theta, \phi)=P(r, \phi, \theta)$ and $\int_{0}^{\pi} P(r, \theta, \phi) \sin ^{2 \lambda} \phi d \phi=1$. Set $f(r, \theta)=\int_{0}^{\pi} P(r, \theta, \phi) f(\phi) \sin ^{2 \lambda} \phi d \phi$. If $1 \leqq p<\infty$, we have by Hölder's inequality:

$$
|f(r, \theta)|^{p} \leqq \int P(r, \theta, \phi)|f(\phi)|^{p} \sin ^{2 \lambda} \phi d \phi
$$

Integrating with respect to $\theta$ and interchanging the order of integration gives $\|f(r, \theta)\|_{p} \leqq\|f\|_{p}, 1 \leqq p<\infty$. The case $p=\infty$ is trivial.

(b) Let $S$ be the linear space of all finite polynomials $\sum_{n=0}^{N} a_{n} P_{n}^{\lambda}(\cos \theta)$. Then $S$ is dense in every $L^{p}\left(d m_{\lambda}\right), 1 \leqq p<\infty$. (In fact $S$ is dense in the space of continuous functions on $[0, \pi]$ in the "sup" topology.) Since clearly $f(r, \theta) \rightarrow f(\theta)$ uniformly and hence in $L^{p}$ for every $f \in S$, part (b) then follows from part (a). Notice also that if $f(\theta)$ is continuous on $[0, \pi]$ then $\|f(r, \theta)-f(\theta)\|_{\infty} \rightarrow 0$.

(c) If $f(\theta)$ is given and in $L^{1}\left(d m_{\lambda}\right)$ it suffices to show that given any $\varepsilon>0$, there exists a set $E_{\varepsilon}$, so that $m_{\lambda}\left(E_{\varepsilon}\right)<\varepsilon$ and $\limsup _{r \rightarrow 1}|f(r, \theta)-f(\theta)|>\varepsilon$ only for $\theta \in E_{\varepsilon}$. 
Thus write $f(\theta)=f_{1}(\theta)+f_{2}(\theta)$ where $f_{1}(\theta) \in S$ and $\left\|f_{2}\right\|_{1}<\delta, \delta$ to be chosen later.

Then $f(r, \theta)-f(\theta)=f_{1}(r, \theta)-f_{1}(\theta)+f_{2}(r, \theta)-f_{2}(\theta)$ and hence $|f(r, \theta)-f(\theta)|$ $\leqq\left|f_{1}(r, \theta)-f_{1}(\theta)\right|+c f_{2}^{*}(\theta)$, since $\left|f_{2}(r, \theta)\right| \leqq C f_{2}^{*}(\theta)$, by Lemma 3 , and $\left|f_{2}(\theta)\right|$ $\leqq f_{2}^{*}(\theta)$ trivially.

Since $\lim \sup _{r \rightarrow 1}\left|f_{1}(r, \theta)-f_{1}(\theta)\right|=0$ for every $\theta$, we must show that $f_{2}^{*}(\theta) \leqq \varepsilon / c$ except in a set of measure $<\varepsilon$. However by Lemma 2 ,

$$
m_{\lambda}\left\{f_{2}^{*}(\theta) \geqq \frac{\varepsilon}{c}\right\} \leqq \frac{2 c}{\varepsilon}\left\|f_{2}\right\|_{1} \leqq \frac{2 c \delta}{\varepsilon} .
$$

Thus we need choose $\delta$ only by $\delta<\varepsilon / 2 c$, which proves (c). (d) is an immediate consequence of the comparison $\sup _{0 \leqq r<1}|f(r, \theta)| \leqq c f^{*}(\theta)$ (Lemma 3 ) and the fact that $\left\|f^{*}\right\|_{p} \leqq A_{p}\|f\|_{p}, 1<p \leqq \infty$ (Lemma 2). This concludes the proof of Theorem 2.

We now come to the converse problem, that of characterizing among all "harmonic" functions those which arise as Poisson integrals of $L^{p}$ functions. For this purpose it is important to recall the space $B$ of Borel measures $d \mu$ on $[0, \pi]$ whose norm

$$
\|d \mu\|=\int_{0}^{\pi}(\sin \theta)^{2 \lambda}|d \mu(\theta)|
$$

is finite.

We define $\int_{0}^{\pi} P(r, \theta, \phi) \sin ^{2 \lambda} \phi d \mu(\phi)$ as the Poisson integral of the measure $d \mu$.

Let $u(x, y)$ be a function defined in the upper semi-disc $x^{2}+y^{2}<1, y>0$. We shall say $u(x, y)$ is $\lambda$-harmonic there (or sometimes "harmonic") if it is of class $C^{2}$ there and satisfies

$$
\Delta_{\lambda}(u)=\frac{\partial^{2} u}{\partial x^{2}}+\frac{\partial^{2} u}{\partial y^{2}}+\frac{2 \lambda}{y} \frac{\partial u}{\partial y}=0
$$

We shall say that $u$ is regular and even if there exists a $C^{2}$ function in the entire disc $x^{2}+y^{2}<1$ which extends $u(x, y)$ (we denote the extension by $u(x, y)$ also) so that $u(x, y)=u(x,-y)$. We notice if $u$ is $\lambda$-harmonic, regular and even, then $\Delta_{\lambda}(u)=0$ also if $y<0$.

We are now in a position to state our characterization of Poisson integrals.

THEOREM 3. Let $u(x, y)$ be $\lambda$-harmonic in the upper semi-disc. Then $u(x, y)=f(r, \theta)$ is the Poisson integral of a function $f(\theta) \in L^{p}\left(d m_{\lambda}\right), 1<p \leqq \infty$, if and only if $u(x, y)$ is regular and even in the disc $x^{2}+y^{2}<1$ and

$$
\sup _{0 \leqq r<1}\|f(r, \theta)\|_{p}<\infty .
$$

For the case $p=1$ we have the following not-unexpected variant. 
THEOREM 3'. Let $u(x, y)$ be $\lambda$-harmonic in the upper semi-disc. Then $u(x, y)=f(r, \theta)$ is the Poisson integral of a measure $d \mu$ so that $\int_{0}^{\pi} \sin ^{2 \lambda} \theta|d \mu(\theta)|<\infty$ if and only if $u(x, y)$ is regular and even and

$$
\sup _{r<1}\|f(r, \theta)\|_{1}<\infty \text {. }
$$

As a corollary we obtain:

COROllary 1. A $\lambda$-harmonic function $u(x, y)$ which is regular, even, and nonnegative is the Poisson integral of a non-negative measure $d \mu$ so that $\int_{0}^{\pi} \sin ^{2 \lambda} \theta d \mu(\theta)<\infty$, and conversely.

As a further consequence, taking into consideration Theorem 2, we have:

COROLlaRY 2. If $u(x, y)$ is $\lambda$-harmonic, regular, even and satisfies

$$
\sup _{0 \leqq r<1}\|u(r \cos \theta, r \sin \theta)\|_{p}<\infty \quad \text { for some } p, 1<p \leqq \infty
$$

then

$$
\lim _{r \rightarrow 1} u(r \cos \theta, r \sin \theta) \text { exists for almost every } \theta .
$$

There are two further conclusions for Poisson integrals that need be mentioned. First, that $\lim _{r \rightarrow 1} u(r \cos \theta, r \sin \theta)$ will exist almost everywhere even for Poisson integrals of measures in $B$. (Theorem 2-(c) covers only absolutely continuous measures.) Second, all limits at the boundary can be taken in the nontangential sense-instead of just the radial sense.

While these facts could be proved by techniques similar to those used above, we prefer postponing consideration of these matters to a later section where they shall follow in the course of the development of $H^{p}$-spaces.

Proof of Theorem 3. We have already observed that if $f(r, \theta)$ is a Poisson, integral it is $\lambda$-harmonic, regular and even. If $f(\theta) \in L^{p}$ then by Theorem 2 , $\|f(r, \theta)\|_{p} \leqq\|f(\theta)\|_{p}$ and thus the necessity is proved. We come therefore to the converse.

For any $r_{0}, 0<r_{0}<1$, write

$$
g(r, \theta)=\int_{0}^{\pi} P(r, \theta, \phi) f\left(r_{0}, \phi\right) d m_{\lambda}(\phi) .
$$

Thus $g(r, \theta)$ is the Poisson integral of the continuous function $f\left(r_{0}, \theta\right)$. Consider now $h(r, \theta)=g(r, \theta)-f\left(r r_{0}, \theta\right)$. It is easy to verify directly that $f\left(r r_{0}, \theta\right)$ is $\lambda$-harmonic (in $(r, \theta)$ ), regular and even in the disc $x^{2}+y^{2}<1$, because $f(r, \theta)$ is. Thus $h(r, \theta)$ has the same properties. Moreover, $h(r, \theta)$ is continuous in the closure $x^{2}+y^{2} \leqq 1$ since $f\left(r r_{0}, \theta\right)$ is, and $g(r, \theta) \rightarrow f\left(r_{0}, \theta\right)$ uniformly as $r \rightarrow 1$. Therefore, in addition, $h(1, \theta)=0$. We claim that $h(r, \theta) \equiv 0$. Assume the contrary, then $h(r, \theta)$ would have a local maximum (or minimum) at an interior point of 
the disc. This contradicts the maximum principle (Theorem 1) and hence $h(r, \theta) \equiv 0$. Thus

$$
f\left(r r_{0}, \theta\right)=\int_{0}^{\pi} P(r, \theta, \phi) f\left(r_{0}, \phi\right) d m_{\lambda}(\phi), \quad 0 \leqq r_{0}, r<1 .
$$

Since $\|f(r, \phi)\|_{p} \leqq M<\infty$, we can select a sequence $r_{1}, r_{2}, \cdots, r_{n}, \cdots$, so that $r_{n} \rightarrow 1$ and $f\left(r_{n}, \theta\right) \rightarrow f(\theta)$ weakly as $r_{n} \rightarrow 1$.

It therefore follows from (6.1) that $f(r, \theta)$ is the Poisson integral of $f(\theta)$, and the theorem is proved.

Theorem $3^{\prime}$ is proved in the same way, except because $\int_{0}^{\pi}|f(r, \theta)| \sin ^{2 \lambda} \theta d \theta$ $\leqq M<\infty$, we now have a sequence $f\left(r_{n}, \theta\right)$ which converges weakly to a measure $d \mu(\theta)$ so that $\int_{0}^{\pi} \sin ^{2 \lambda} \theta|d \mu(\theta)|<\infty$.

The proof of Corollary 1 is based on the following fact: If $u(x, y)=f(r, \theta)$ is $\lambda$-harmonic, regular, and even, then

$$
u(0,0) \int_{0}^{\pi}(\sin \theta)^{2 \lambda} d \theta=\int_{0}^{\pi} u(r \cos \theta, r \sin \theta)(\sin \theta)^{2 \lambda} d \theta
$$

This is the mean value property, which in this context is due to Weinstein [23]. (6.2) also follows immediately from a more general inequality (3.1) for "subharmonic" functions. In any case, if $u(x, y) \geqq 0$, then (6.2) implies that $u(x, y)=f(r, \theta)$ satisfies $\sup _{r<1} \int_{0}^{\pi}|f(r, \theta)| \sin ^{2 \lambda} \theta d \theta<\infty$. Theorem $3^{\prime}$ is therefore applicable.

Corollary 2 is an immediate consequence of Theorems 2 and 3.

7. The conjugate kernel. Let us recall briefly the definitions of conjugacy introduced above.

If

$$
f(\theta) \sim \sum a_{n} P_{n}^{\lambda}(\cos \theta)
$$

and

$$
f(r, \theta)=\sum a_{n} r^{n} P_{n}^{\lambda}(\cos \theta), \quad r<1,
$$

then

$$
f(r, \theta)=2 \lambda \sum_{n=1}^{\infty} \frac{a_{n}}{n+2 \lambda} r^{n} \sin \theta P_{n-1}^{\lambda+1}(\cos \theta), \quad 0 \leqq \theta \leqq \pi .
$$

If we set $u(x, y)=f(r, \theta)$ and $v(x, y)=y^{2 \lambda} f(r, \theta)$ then $u$ and $v$ satisfy the CauchyRiemann equations $v_{x}=-y^{2 \lambda} u_{y}$, and $v_{y}=y^{2 \lambda} u_{x}$ and their analogues in polar coordinates: $v_{r}=-\left((r \sin \theta)^{2 \lambda} / r\right) u_{\theta}, v_{\theta}=r(r \sin \theta)^{2 \lambda} u_{r}$. That these equations are verified follows easily from the relation $(d / d t) P_{n}^{\lambda}(t)=2 \lambda P_{n-1}^{\lambda+1}(t)$, see Szegö $[18$, p. 83]. 
Let us now notice that

where

$$
\tilde{f}(r, \theta)=\int_{0}^{\pi} Q(r, \theta, \phi) f(\phi) \sin ^{2 \lambda} \phi d \phi,
$$

$$
Q(r, \theta, \phi)=\sum_{n=1}^{\infty} \frac{2 \lambda}{n+2 \lambda} r^{n} \gamma_{n} \sin \theta P_{n-1}^{\lambda+1}(\cos \theta) P_{n}^{\lambda}(\cos \phi)
$$

and where $\gamma_{n}$ are the normalizing factors (2.9). $Q$ will be called the conjugate Poisson kernel.

In view of the equations $v_{r}=-\left((r \sin \theta)^{2 \lambda} / r\right) u_{\theta}, v=y^{2 \lambda} \tilde{f}(r, \theta)$, and $Q(0, \theta, \phi)$ $=0$, we obtain

$$
Q(r, \theta, \phi)=-r^{-2 \lambda} \int_{0}^{r} t^{2 \lambda-1} P_{\theta}(t, \theta, \phi) d t,
$$

where $P$ is the Poisson kernel(2.11). Equation(7.3)allows us to reduce the problem of analyzing the kernel $Q$ in terms of the kernel $P$, for which we have an explicit formula. The result of this analysis is as follows.

LEMMA 4. If $0 \leqq \theta \leqq \pi, 0 \leqq \phi \leqq \pi / 2,0<\lambda$, then

$$
\begin{aligned}
Q(r, \theta, \phi)= & O\left((\sin \phi)^{-2 \lambda-1}\right) \quad \text { if } 2 \theta<\phi, \\
= & O\left((\sin \theta)^{-2 \lambda-1}\right) \quad \text { if } \frac{1}{2} \theta>\phi, \\
Q(r, \theta, \phi)= & c_{\lambda} r^{\lambda}(\sin \theta \sin \phi)^{-\lambda} \frac{\sin (\theta-\phi)}{1-2 r \cos (\theta-\phi)+r^{2}} \\
& +O\left[(\sin \theta)^{-2 \lambda-1}\left(1+\log ^{+}\left(\frac{\sin \theta \sin \phi}{1-\cos (\theta-\phi)}\right)\right)\right] \text { if } \frac{\theta}{2} \leqq \phi \leqq 2 \theta .
\end{aligned}
$$

REMARKS. It follows from (7.2) that $Q(r, \theta, \phi)=Q\left(r, \theta^{\prime}, \phi^{\prime}\right)$, where $\theta^{\prime}=\pi-\theta$, $\phi^{\prime}=\pi-\phi$ (because $\left.P_{n}(-t)=(-1)^{n} P_{n}(t)\right)$. Thus very similar estimates hold for $Q$ in the range $\pi / 2 \leqq \phi \leqq \pi$. The lemma shows that except for the factor $c_{\lambda} r^{\lambda}(\sin \theta \sin \phi)^{-\lambda}$, the kernel $Q$ behaves like the conjugate kernel in the trigonometric case for $\theta$ near $\phi$.

Proof. Let us define $D$ by

$$
D=1-2 r(\cos \theta \cos \phi+\sin \theta \sin \phi \cos t)+r^{2} \text {. }
$$

We then have

$$
P(r, \theta, \phi)=(\lambda / \pi)\left(1-r^{2}\right) \int_{0}^{\pi} \frac{\sin ^{2 \lambda-1} t}{D^{\lambda+1}} d t .
$$

Differentiation of both sides establishes the elementary identity

$$
\int_{0}^{r} \frac{t^{2 \lambda}\left(1-t^{2}\right)}{\left(1-2 a t+t^{2}\right)^{\lambda+2}} d t=\frac{r^{2 \lambda+1}}{(\lambda+1)\left(1-2 a r+r^{2}\right)^{\lambda+2}}-\frac{\lambda}{\lambda+1} \int_{0}^{r} \frac{t^{2 \lambda}}{\left(1-2 a t+t^{2}\right)^{\lambda+1}} d t .
$$


As we saw before

$$
Q(r, \theta, \phi)=-r^{-2 \lambda} \int_{0}^{r} t^{2 \lambda-1} P_{\theta}(t, \theta, \phi) d t .
$$

But

$$
P_{\theta}(t, \theta, \phi)=-\frac{(\lambda+1) \lambda}{\pi}\left(1-t^{2}\right) t \int_{0}^{\pi} \frac{b(\sin \tau)^{2 \lambda-1}}{\left(1-2 a t+t^{2}\right)^{\lambda+1}} d \tau
$$

where

$$
\begin{aligned}
& a=\cos \theta \cos \phi+\sin \theta \sin \phi \cos \tau, \\
& b=2 \sin \theta \cos \phi-2 \cos \theta \sin \phi \cos \tau .
\end{aligned}
$$

Substituting in the above identity gives

$$
Q(r, \theta, \phi)=-r^{-2 \lambda+1} R(r, \theta, \phi)+\lambda r^{-2 \lambda} \int_{0}^{r} R(t, \theta, \phi) d t,
$$

with

$$
R(r, \theta, \phi)=\frac{-\lambda}{\pi} r^{2 \lambda-1} \int_{0}^{r} D_{\theta} \cdot D^{-\lambda-1} \sin ^{2 \lambda-1} t d t .
$$

Thus it suffices to obtain similar estimates for the kernel $R(r, \theta, \phi)$.

Consider first the case, $\phi>2 \theta$. Now

since $\phi>2 \theta$.

$$
\begin{aligned}
D^{\lambda+1} & \geqq\left((1-r)^{2}+2 r(1-\cos (\theta-\phi))\right)^{\lambda+1} \geqq c(1-\cos (\theta-\phi))^{\lambda+1} \\
& \geqq c|\sin (\theta-\phi)|^{2 \lambda+2} \geqq c(\sin \phi)^{2 \lambda+2},
\end{aligned}
$$

However, $\left|D_{\theta}\right|=2 r|\sin \theta \cos \phi-\cos \theta \sin \phi \cos t| \leqq c \sin \phi($ since $\phi>2 \theta)$, we therefore have by substituting these estimates in (7.4)

$$
|R(r, \theta, \phi)| \leqq c(\sin \phi)^{-2 \lambda-1} \quad \text { for } \phi>2 \theta, 0 \leqq \theta \leqq \pi .
$$

By an almost identical argument

$$
|R(r, \theta, \phi)| \leqq c(\sin \theta)^{-2 \lambda-1}, \quad \frac{1}{2} \theta>\phi, 0 \leqq \theta \leqq \pi .
$$

We now consider the more critical range, $\theta / 2 \leqq \phi \leqq 2 \theta$.

Write,

$$
\begin{aligned}
R(r, \theta, \phi) & =\frac{-\lambda r^{2 \lambda-1}}{\pi} \int_{0}^{\pi} D_{\theta} D^{-\lambda-1} \sin ^{2 \lambda-1} t d t \\
(7.7) & =\frac{-\lambda r^{2 \lambda-1}}{\pi} \int_{0}^{\pi / 2} D_{\theta} D^{-\lambda-1} \sin ^{2 \lambda-1} t d t+\frac{-\lambda r^{2 \lambda-1}}{\pi} \int_{\pi / 2}^{\pi} D_{\theta} D^{-\lambda-1} \sin ^{2 \lambda-1} t d t \\
& =R_{1}(r, \theta, \phi)+R_{2}(r, \theta, \phi) .
\end{aligned}
$$


In $R_{1}$ the behavior near $t=0$ will be decisive and in $R_{2}$ the behavior near $t=\pi$ will be critical. Thus in $R_{1}$ we shall systematically use the fact that $\cos t=1-t^{2} / 2+O\left(t^{4}\right)$, and $\sin ^{2 \lambda-1} t=t^{2 \lambda-1}+O\left(t^{2 \lambda+1}\right)$, as $t \rightarrow 0$; similar facts are used when dealing with $R_{2}$, whose contribution is solely to the error term.

Now

$$
\begin{aligned}
D^{\lambda+1} & =\left[1-2 r(\cos \theta \cos \phi+\sin \theta \sin \phi \cos t)+r^{2}\right]^{\lambda+1} \\
& =\left[1-2 r \cos (\theta-\phi)+r^{2}+r t^{2} \sin \theta \sin \phi+O\left(t^{4}\right) r \sin \theta \sin \phi\right]^{\lambda+1} .
\end{aligned}
$$

Since $1-2 r \cos (\theta-\phi)+r^{2} \geqq 0$, and $r \sin \theta \sin \phi \geqq 0$, we get by the mean value theorem

$$
\begin{aligned}
D^{-\lambda-1}= & {\left[1-2 r \cos (\theta-\phi)+r^{2}+t^{2} r \sin \theta \sin \phi\right]^{-\lambda-1} } \\
& +O\left(t^{4}\right) r \sin \theta \sin \phi \cdot D^{-\lambda-2} .
\end{aligned}
$$

Also

$$
D_{\theta}=2 r \sin (\theta-\phi)+O\left(t^{2}\right) \sin \phi
$$

since $\theta / 2 \leqq \phi \leqq 2 \theta, 0 \leqq \theta \leqq \pi$. Inserting the estimates (7.8) and (7.9) in (7.7), simplifying, and using the fact that $t^{2} r \sin \theta \sin \phi \leqq c D$, we get

$$
R_{1}(r, \theta, \phi)=\frac{-\lambda}{\pi} r^{2 \lambda-1} \int_{0}^{\pi / 2} \frac{t^{2 \lambda+1} \sin (\theta-\phi)}{\left[1-2 r \cos (\theta-\phi)+r^{2}+r t^{2} \sin \theta \sin \phi\right]^{\lambda+1}} d t
$$

$$
+O(\sin \phi) \int_{0}^{\pi / 2} t^{2 \lambda+1}\left[1-2 r \cos (\theta-\phi) t r^{2}+r t^{2} \sin \theta \sin \phi\right]^{-\lambda-1} d t .
$$

We handle these integrals by making the substitution

$$
u=\left(\frac{r \sin \theta \sin \phi}{1-2 r \cos \left(\theta-\phi+r^{2}\right.}\right)^{1 / 2} t=\left(\frac{\sigma}{\Delta}\right)^{1 / 2} t,
$$

as in the proof of Lemma 1 of $\S 4$. (Here, $\sigma=r \sin \theta \sin \phi$, and $\left.\Delta=1-2 r \cos (\theta-\phi)+r^{2}.\right)$

Thus the first integral in (7.10) becomes

$$
\frac{-\lambda}{\pi} r^{2 \lambda-1} \frac{\sin (\theta-\phi)}{\Delta} \sigma^{-\lambda} \int_{0}^{(\sigma / \Delta)^{1 / 2}(\pi / 2)} \frac{u^{2 \lambda-1}}{\left(1+u^{2}\right)^{\lambda+1}} d u .
$$

However

$$
\int_{0}^{\xi} \frac{u^{2 \lambda-1}}{\left(1+u^{2}\right)^{\lambda+1}} d u=\int_{0}^{\infty} \frac{u^{2 \lambda-1}}{\left(1+u^{2}\right)^{\lambda+1}} d u+O\left(1 / \xi^{2}\right) \quad \xi \rightarrow \infty .
$$


Therefore the first integral in (7.10) becomes

$$
c_{\lambda} r^{\lambda-1}(\sin \theta \sin \phi)^{-\lambda} \frac{\sin (\theta-\phi)}{1-2 r \cos (\theta-\phi)+r^{2}}+O\left(|\sin (\theta-\phi)| \sigma^{-\lambda-1}\right),
$$

and this equals

$$
c_{\lambda} r^{\lambda-1}(\sin \theta \sin \phi)^{-\lambda} \frac{\sin (\theta-\phi)}{1-2 r \cos (\theta-\phi)+r^{2}}+O(\sin \theta)^{-2 \lambda-1},
$$

since $\theta / 2 \leqq \phi \leqq 2 \theta$.

The second integral in (7.10) is dealt with similarly, and leads to the estimate

$$
O\left(\sin \phi \cdot \sigma^{-\lambda-1}\left[1+\log ^{+}\left(\frac{\sigma}{\Delta}\right)\right]\right) \text {. }
$$

Since $\sin \phi \geqq c \sin \theta$ and $\Delta \geqq c[1-\cos (\theta-\phi)]$, in this range the above $O$ term is

$$
O(\sin \theta)^{-2 \lambda-1}\left(1+\log ^{+}\left(\frac{\sin \theta \sin \phi}{1-\cos (\theta-\phi)}\right)\right) \text {. }
$$

Hence (7.11) and (7.12) combined give us the required estimates for $R_{1}$ and thus for $R$, and finally for $Q$ in the range $\theta / 2 \leqq \phi \leqq 2 \theta$. This concludes the proof of the lemma.

8. $L^{p}$ theorems for conjugate functions. Let us recall the definition (7.1) of the conjugate harmonic function $\tilde{f}(r, \theta)$ :

$$
f(r, \theta)=2 \lambda \sum_{n=1}^{\infty} \frac{a_{n}}{n+2 \lambda} r^{n} \sin \theta P_{n-1}^{\lambda+1}(\cos \theta), \quad 0 \leqq r<1,
$$

where

$$
f(\theta) \sim \sum a_{n} P_{n}^{\lambda}(\cos \theta)
$$

We shall need also the $L^{p}$ classes with norm $\|f\|_{p}=\left(\int_{0}^{\pi}|f(\phi)|^{p} d m_{\lambda}(\phi)\right)^{1 / p}$, where $d m_{\lambda}(\phi)=(\sin \phi)^{2 \lambda} d \phi$.

We come now to one of our main results-an extension of the M. Riesz theorem for conjugate functions for the $L^{p}$ norm, and of Kolmogoroff's inequality for $L^{1}$.

THEOREM 4. (a) If $f(\theta) \in L^{p}\left(d m_{\lambda}\right), 1<p<\infty$, then $\|\tilde{f}(r, \theta)\|_{p} \leqq A_{p}\|f\|_{r}$.

(b) If $f \in L^{1}\left(d m_{\lambda}\right), s>0$, and $E_{s}=\{\theta|| f(r, \theta) \mid>s\}$. Then

$$
m_{\lambda}\left(E_{s}\right) \leqq(A / s)\|f\|_{1} \text {. }
$$

Proof. We consider, for fixed $r, 0 \leqq r<1$, simultaneously two operators, $T_{r}$ and $T^{*}$ defined by

$$
\left(T_{r} f\right)(\theta)=\int_{0}^{\pi} Q(r, \theta, \phi) f(\phi) d m_{\lambda}(\phi)
$$

and 


$$
\left(T_{r}^{*} f\right)(\theta)=\int_{0}^{\pi}(r, \phi Q, \theta) f(\phi) d m_{\lambda}(\phi) .
$$

Thus $T_{r}(f)(\theta)=\tilde{f}(r, \theta)$, and $T_{*}^{*}$ is the adjoint of $T_{r}$. We prove first

$$
\left\|T_{r} f\right\|_{2} \leqq\|f\|_{2} \text {. }
$$

In fact,

where

$$
\left\|T_{r} f\right\|_{2}^{2}=(2 \lambda)^{2} \sum_{n=1}^{\infty} \frac{\left|a_{n}\right|^{2} r^{2 n}}{(n+2 \lambda)^{2}}\left(\gamma_{n}^{*}\right)^{-1}
$$

$$
\left(\gamma_{n}^{*}\right)^{-1}=\int_{0}^{\pi}\left[P_{n-1}^{\lambda+1}(\cos \theta)\right]^{2} d m_{\lambda+1}(\theta)=2^{-1-2 \lambda} \pi(\Gamma(\lambda+1))^{-2} \frac{\Gamma(n+2 \lambda+1)}{(n+\lambda)(n-1) !} .
$$

However,

where

$$
\|f\|_{2}^{2}=\Sigma\left|a_{n}\right|^{2} \gamma_{n}^{-1}
$$

$$
\gamma_{n}^{-1}=\int_{0}^{\pi}\left[P_{n}^{\lambda}(\cos \theta)\right]^{2} d m_{\lambda}(\theta)=2^{1-2 \lambda} \pi(\Gamma(\lambda))^{-2} \frac{\Gamma(n+2 \lambda)}{(n+\lambda) n !}
$$

(see equation (2.9)).

Thus (8.3) is equivalent with $\sup _{n}(2 \lambda)^{2}(n+2 \lambda)^{-2}\left(\gamma_{n} / \gamma_{n}^{*}\right) \leqq 1$, which after reduction becomes $\sup _{n}(n /(n+2 \lambda)) \leqq 1$, and this proves (8.3).

By a standard duality argument we then obtain

$$
\left\|T_{r}^{*} f\right\|_{2} \leqq\|f\|_{2} \text {. }
$$

We have therefore proved part (a) of the theorem in the special case $p=2$. In order to make further progress we must now prove the "weak-type" estimate, that is part (b) of the theorem. We shall prove this for both $T_{r}$ and $T_{r}^{*}$. In so doing we shall systematically use the following remark: If finitely many functions satisfy the weak-type estimates arising in (b) for all $s>0$, then so do their linear combinations with constant coefficients, with possibly larger constant.

We now make a few simplifying assumptions. First we take $f \geqq 0$; next we assume that $f(\phi)=0$, if $\pi / 2 \leqq \phi \leqq \pi$. The case when the support of $f$ lies in $(\pi / 2, \pi)$ is dealt with similarly.

Then

$$
\begin{aligned}
\left(T_{r} f\right)(\theta)= & \int_{0}^{\pi / 2} Q(r, \theta, \phi) f(\phi) d m_{\lambda}(\phi) \\
= & \int_{0}^{\theta / 2} Q(r, \theta, \phi) f(\phi) d m_{\lambda}(\phi)+\int_{2 \theta}^{\pi / 2} Q(r, \theta, \phi) f(\phi) d m_{\lambda}(\phi) \\
& +\int_{\theta / 2}^{2 \theta} Q(r, \theta, \phi) f(\phi) d m_{\lambda}(\phi)
\end{aligned}
$$


Let us set

$$
\begin{aligned}
& M(f)(\theta)=(\sin \theta)^{-2 \lambda-1} \int_{0}^{\pi / 2} f(\phi) d m_{\lambda}(\phi) \\
& N(f)(\theta)=(\sin \theta)^{-2 \lambda-1} \int_{\theta / 2}^{2 \theta} \log ^{+} \frac{\sin \theta \sin \phi}{1-\cos (\theta-\phi)} f(\phi) d m_{\lambda}(\phi)
\end{aligned}
$$

and

$$
Q_{0}(r, \theta, \phi)=c_{\lambda} r^{\lambda} \frac{\sin ^{-\lambda} \theta \sin ^{-\lambda} \phi \sin (\theta-\phi)}{1-2 r \cos (\theta-\phi)+r^{2}} .
$$

Then by virtue of Lemma 4 we have, after a simple reduction,

$$
\left|T_{r}(f)(\theta)-\int_{\theta / 2}^{2 \theta} Q_{0}(r, \theta, \phi) f(\phi) d m_{\lambda}(\phi)\right| \leqq c[M(f)(\theta)+N(f)(\theta)] .
$$

It is easily seen that

$$
m_{\lambda}(\theta \mid M f(\theta)>s) \leqq \frac{c}{s} \int_{0}^{\pi / 2} f d m_{\dot{\lambda}}(\phi)
$$

Next we claim that

$$
m_{\lambda}(\theta \mid N f(\theta)>s) \leqq \frac{c}{s} \int_{0}^{\pi / 2} f d m_{\lambda} .
$$

This will follow if we show that $\int_{0}^{\pi} N f(\theta) d m_{\lambda}(\theta) \leqq c \int_{0}^{\pi} f(\phi) d m_{\lambda}(\phi)$. In fact

$$
\begin{aligned}
\int_{0}^{\pi} N f(\theta) d m_{\lambda}(\theta) & =\int_{0}^{\pi}(\sin \theta)^{-1}\left\{\int_{\theta / 2}^{2 \theta} f(\phi) \log ^{+} \frac{\sin \theta \sin \phi}{1-\cos (\theta-\phi)} d m_{\lambda}(\phi)\right\} d \theta \\
& =\int_{0}^{\pi / 2} f(\phi) K(\phi) d m_{\lambda}(\phi)
\end{aligned}
$$

where

$$
K(\phi)=\int_{\phi / 2}^{2 \phi} \log ^{+}\left(\frac{\sin \theta \sin \phi}{1-\cos (\theta-\phi)}\right) \frac{d \theta}{\sin \theta}, \quad 0 \leqq \phi \leqq \pi / 2 .
$$

However, a simple reduction shows, that in this range

$$
K(\phi) \leqq A \int_{\phi / 2}^{2 \phi} \log ^{+}\left(\frac{\theta \phi}{(\theta-\phi)^{2}}\right) \frac{d \theta}{\theta}+B=A \int_{1 / 2}^{2} \log ^{+}\left(\frac{\theta}{(\theta-1)^{2}}\right) \frac{d \theta}{\theta}+B .
$$

Thus $K(\phi)$ is bounded and therefore we obtain (8.9).

We now come to the main contribution-that given by the kernel $Q_{0}(r, \theta, \phi)$ - 
which is the principal term of $Q(r, \theta, \phi)$. For this purpose consider the sets $E_{n}=\left(2^{-n-1} \pi, 2^{-n} \pi\right)$ and the sets $E_{n}^{\prime}=\left(2^{-n-2} \pi, 2^{-n+1} \pi\right)$. In the integral

$$
\int_{\theta / 2}^{2 \theta} Q_{0}(r, \theta, \phi) f(\phi) d m_{\lambda}(\phi)
$$

if $\theta$ lies in the set $E_{n}$, the integration in $\phi$ ranges only over part of $E_{n}^{\prime}$. Thus if $\chi_{E_{n}^{\prime}}$ is the characteristic function of $E_{n}^{\prime}$, the integral of (8.10) is identical with the sum

$$
\sum_{n=0}^{\infty} \chi_{E_{n}}(\theta) \int_{\theta / 2}^{2 \theta} Q_{0}(r, \theta, \phi) f_{i z}(\phi) d m_{\lambda}(\phi)
$$

where

$$
f_{n}(\phi)=f(\phi) \chi_{E_{n}^{\prime}}(\phi) .
$$

We would like, however, to compare the sum (8.11) with the sum

$$
\sum_{n=0}^{\infty} \chi_{E_{n}}(\theta) \int_{E_{n}^{\prime}} Q_{0}(r, \theta, \phi) f(\phi) d m_{\lambda}(\phi) \text {. }
$$

The difference of the $n$th terms of these two sums is dominated by

where

$$
\chi_{E_{n}}(\theta) \int_{F_{n}}\left|Q_{0}(r, \theta, \phi)\right| f(\phi) d m_{\lambda}(\phi),
$$

$$
F_{n}=\left(2^{-n-2} \pi, \theta / 2\right) \cup\left(2 \theta, 2^{-n+1} \pi\right), \quad \theta \in E_{n} .
$$

However, in this range it is easily verified that $\left|Q_{0}(r, \theta, \phi)\right| \leqq c(\sin \theta)^{-2 \lambda-1}$. Thus it follows that the difference of the sums (8.11) and (8.12) is dominated by

$$
c(\sin \theta)^{-2 \lambda-1} \sum_{n=0}^{\infty} \int_{E_{n}^{\prime}} f(\phi) d m_{\lambda} \leqq c^{\prime}(\sin \theta)^{-2 \lambda-1} \int_{0}^{\pi} f(\phi) d m_{\lambda}(\phi) .
$$

This is of the form $M f$ (see (8.5)), and leads to the desired estimates. We have therefore finally reduced the problem to that of the sum (8.12).

Let then $E^{s}$ denote the set of $\theta$ 's for which the absolute value of the sum (8.12) exceeds $s$. Then

$$
E^{s}=\bigcup_{n=0}^{\infty}\left(E^{s} \cap E_{n}\right), \text { and } m_{\lambda}\left(E^{s}\right)=\sum_{n=0}^{\infty} m_{\lambda}\left(E^{s} \cap E_{n}\right) .
$$

We deal with the set $E^{s} \cap E_{0}$ separately. Therefore we take $n \geqq 1$. It is clear that on $E_{n},(\sin \theta)^{-\lambda}$ is of the order $2^{+n \lambda}$; thus

$$
E^{s} \cap E_{n} \subset\left\{\theta|| \int_{E_{n}^{\prime}} \frac{(\sin \phi)^{-\lambda} \sin (\theta-\phi)}{1-2 r \cos (\theta-\phi)+r^{2}} f(\phi) d m_{\lambda}(\phi) \mid>c 2^{-n \lambda} s\right\} .
$$

Thus by the classical theorem on conjugate functions for trigionometric series 


$$
\begin{aligned}
\int_{E^{s} \cap E_{n}} d \theta & \leqq c^{\prime} \frac{2^{+n \lambda}}{s} \int_{E_{n}^{\prime}} f(\phi) \sin ^{\lambda} d \phi \\
& \leqq C^{\prime} \frac{2^{+n \lambda}}{s} \cdot 2^{+n \lambda} \int_{E_{n}^{\prime}} f(\phi) \sin ^{2 \lambda} \phi d \phi .
\end{aligned}
$$

However,

$$
m_{\lambda}\left(E^{s} \cap E_{n}\right)=\int_{E^{s} \cap E_{n}} \sin ^{2 \lambda} d \theta \leqq C 2^{-2 n \lambda} \int_{E^{s \cap E_{n}}} d \theta
$$

Thus,

$$
\sum_{n=1}^{\infty} m_{\lambda}\left(E^{s} \cap E_{n}\right) \leqq C^{\prime} / s \sum_{n=1}^{\infty} \int_{E_{n}^{\prime}} f(\phi) d m_{\lambda} \leqq C / s \int_{0}^{\pi} f(\phi) d m_{\lambda}(\phi) .
$$

It remains to consider $E^{s} \cap E_{0}$. Now $E_{0}=(\pi / 2, \pi)=(\pi / 2,(3 / 4) \pi) \cup[(3 / 4) \pi, \pi)$ $=E_{0}^{(1)} \cup E_{0}^{(2)}$. The measure $m_{\lambda}\left(E^{s} \cap E_{0}^{(1)}\right) \leqq(C / s) \int_{0}^{\pi} f(\phi) d m_{\lambda}$ by the same arguments used to consider $m_{\lambda}\left(E^{s} \cap E_{n}\right), n \geqq 1$. Finally, when $\theta \in[(3 / 4) \pi, \pi], \phi \in[0, \pi / 2]$ we use the trivial estimate $\left|Q_{0}(r, \theta, \phi)\right| \leqq C(\sin \theta)^{-2 \lambda-1}$, which reduces the estimate of the measure $m_{\lambda}\left(E^{s} \cap E_{0}^{(2)}\right)$ to the integral $M(f)(\theta)$ considered earlier.

Combining these estimates, and taking into consideration the remarks made earlier, we obtain

$$
m_{\lambda}\left(\theta \mid\left(T_{r} f(\theta) \mid>s\right) \leqq A / s\|f\|_{1} .\right.
$$

This proves part (b) of the theorem.

Since we have essentially identical estimates for the kernels $Q(r, \phi, \theta)$ and $Q(r, \theta, \phi)$ we also obtain

$$
m_{\lambda}\left(\theta|| T_{r}^{*} f(\theta) \mid>s\right) \leqq A / s \cdot\|f\|_{1} .
$$

A combination of (8.13) and (8.3) via the Marcinkiewicz interpolation theorem $\left({ }^{5}\right)$ proves

$$
\left\|T_{r}(f)\right\|_{p} \leqq A_{p}\|f\|_{p}, \quad 1<p \leqq 2 .
$$

Similarly, (8.14) and (8.4) lead to

$$
\left\|T_{r}^{*}(f)\right\|_{p} \leqq A_{p}\|f\|_{p}, \quad 1<p \leqq 2 .
$$

However, by a standard duality argument (8.16) gives

$$
\left\|T_{r}(f)\right\|_{q} \leqq A_{q}\|f\|_{q}, \quad 2 \leqq q<\infty .
$$

Therefore the theorem is completely proved.

(5) See Zygmund [26, Vol. II, p. 111]. 
CoRollary 1. Let $f \in L^{p}\left(d m_{\lambda}\right), 1<p<\infty$; then $\tilde{f}(r, \theta)$ converges in $L^{p}=$ norm to $\tilde{f}(\theta)$, as $r \rightarrow 1$, and

$$
\|\tilde{f}\|_{p} \leqq A_{p}\|f\|_{p}
$$

Moreover,

$$
\tilde{f}(\theta) \sim 2 \lambda \sum \frac{a_{n}}{n+2 \lambda}(\sin \theta) P_{n-1}^{\lambda+1}(\cos \theta) .
$$

Corollary 2. If $d \mu$ is a measure belonging to $B, d \mu \sim \sum a_{n} P_{n}^{\lambda}(\cos \theta)$ and

then

$$
\tilde{f}(r, \theta)=2 \lambda \sum_{n=1}^{\infty} \frac{a_{n}}{n+2 \lambda} r^{n} \sin \theta P_{n-1}^{\lambda+1}(\cos \theta) \text {, }
$$

$$
\left(\int_{0}^{\pi}|\tilde{f}(r, \theta)|^{p} d m_{\lambda}(\theta)\right)^{1 / p} \leqq A_{p}\|d \mu\|, \quad 0<p<1 .
$$

Corollary 1 is proved in a familiar way from part (a) of the theorem. To prove Corollary 2, let $0<r_{0}<1$, and let $g(\theta)=f\left(r_{0}, \theta\right)$, where $f\left(r_{0}, \theta\right)$ is the Poisson integral of the measure $d \mu$. Then $\left\|f\left(r_{0}, \theta\right)\right\|_{1} \leqq\|d \mu\|$. Now apply part (b) of the theorem to $g$ and let $r_{0} \rightarrow 1$.

We see from the theorem and its corollary that the conjugate mapping, $f \rightarrow \tilde{f}$ is bounded on $L^{p}, 1<p<\infty$, and satisfies an appropriate substitute relation for $L^{1}$. This brings us naturally to the problem of the inverse mapping. Let us consider, for the moment, certain facts about the conjugate mapping, $f \rightarrow \tilde{f}$, in the classical trigonometric case. The adjoint of the mapping $f \rightarrow \tilde{f}$ is the mapping $f \rightarrow-\tilde{f}$; while the inverse of the mapping $f \rightarrow \tilde{f}$ (defined on those $f$ without constant term) is again the mapping $f \rightarrow-\tilde{f}$. However, in the general case we consider, this is no longer true. Thus we had to consider side-by-side with the mapping $T_{r}$ the mapping $T_{r}^{*}$, in our proof of Theorem 4 .

To study the inverse mapping, define the operator $S_{r}$ by

$$
S_{r}(f)(\theta)=(2 \lambda)^{-1} \sum_{n=1}^{\infty} a_{n}(n+2 \lambda) r^{n} P_{n}^{\lambda}(\cos \theta)
$$

whenever

It follows that

$$
f(\theta) \sim \sum_{n=1}^{\infty} a_{n}(\sin \theta) P_{n-1}^{\lambda+1}(\cos \theta)
$$

where

$$
S_{r}(f)(\theta)=\int_{0}^{\pi} Q_{-}(r, \theta, \phi) f(\phi) d m_{\lambda}(\phi),
$$

$$
Q_{-}(r, \theta, \phi)=(2 \lambda)^{-1} \sum_{n=1}^{\infty}(n+2 \lambda) \gamma_{n}^{*} r^{n} P_{n}^{\lambda}(\cos \theta) \sin \phi P_{n-1}^{\lambda+1}(\cos \phi)
$$


(For the definition of the constants $\gamma_{1 n}^{*}$ see the formulae following (8.3).)

If we recall the formula for $Q(r, \theta, \phi)$ (see (7.2)) and the fact that

we get

$$
(2 \lambda)^{-2}(n+2 \lambda)^{2} \frac{\gamma_{n}^{*}}{\gamma_{n}}=\frac{n+2 \lambda}{n}=1+\frac{2 \lambda}{n}
$$

Thus,

$$
Q_{-}(r, \theta, \phi)=Q(r, \phi, \theta)+2 \lambda \int_{0}^{r} t^{-1} Q(t, \phi, \theta) d t
$$

$$
S_{r}=T_{r}^{*}+2 \lambda \int_{0}^{r} t^{-1} T_{t}^{*} d t
$$

However, we have seen that $\left\|T_{r} f\right\|_{p} \leqq A_{p}\|f\|_{p}, 1<p<\infty$, and thus $\left\|T_{r}^{*} f\right\|_{p} \leqq A_{p}\|f\|_{p}, 1<p<\infty$. From (8.19) it then follows that

$$
\left\|S_{r}(f)\right\|_{p} \leqq A_{p}\|f\|_{p}, \quad 1<p<\infty, \quad 0 \leqq r<1 .
$$

Incidentally, we have used the semitrivial estimate $\left\|T_{r}^{*} f\right\|_{p} \leqq A r\|f\|_{p}$ if $0 \leqq r \leqq 1 / 2$.

When $f$ is, say, a polynomial,

$$
f(\theta)=\sum_{n=1}^{N} a_{n} P_{n}^{\lambda}(\cos \theta), \quad \int_{0}^{\pi} f(\theta) d m_{\lambda}(\theta)=0,
$$

then it follows immediately from the definitions of $f$ and $S_{r}$ that $\lim _{r \rightarrow 1} S_{r}(f)(\theta)$ $=f(\theta)$ in $L^{p}$-norm.

Combining this with (8.20) gives the following further corollary.

Corollary 3. If $f(\theta) \in L^{p}\left(d m_{\lambda}\right), 1<p<\infty$, and

$$
\int_{0}^{\pi} f(\theta) d m_{\lambda}(\theta)=0
$$

then

$$
B_{p}\|f\|_{p} \leqq\|\tilde{f}\|_{p} \leqq A_{p}\|f\|_{p} .
$$

9. Theory of $H^{p}$-spaces: Harmonic majorization. Let $u$ and $v$ be a pair of conjugate harmonic functions on the upper semi-disc, that is

there.

$$
v_{x}=-y^{2 \lambda} u_{y} \text { and } v_{y}=y^{2 \lambda} u_{x}
$$

We have studied in $\S 6$ the conditions under which $u(x, y)$ can be represented as a Poisson integral of boundary values taken on the perimeter of the upper semi-disc. Whenever this is possible we have

$$
u(x, y)=f(r, \theta)=\sum_{n=0}^{\infty} a_{n} r^{n} P_{n}^{\lambda}(\cos \theta), \quad(x, y)=(r \cos \theta, r \sin \theta) .
$$


$u(x, y)$ then has the additional property that it can be extended past the singular line $y=0$ to the whole disc so that it is even in $y$ and regular there. For $u$ 's represerted as in (9.1) we can write down a similar series representing the conjugate $v$,

$$
v(x, y)=y^{2 \lambda} \tilde{f}(r, \theta)=y^{2 \lambda} \cdot 2 \lambda \sum_{n=1}^{\infty} \frac{a_{n} r^{n}}{n+2 \lambda} \sin \theta P_{n-1}^{\lambda+1}(\cos \theta) .
$$

It is to be noted that $\tilde{f}(r, \theta)$ can also be extended past the line $y=0$, so that as a function of $x$ and $y$ it is odd in $y$, and regular in the entire disc.

In view of the $L^{p}$ boundedness results obtained in $\S 8$ it is natural to consider $f(r, \theta)$ and $\tilde{f}(r, \theta)$ on the same footing-as opposed to $u$ and $v$. For this reason it is convenient to use adjusted functions $U$ and $V$ defined by

$$
\begin{aligned}
& U(x, y)=u(x, y)=f(r, \theta), \\
& V(x, y)=y^{-2 \lambda} v(x, y)=\tilde{f}(r, \theta) .
\end{aligned}
$$

We shall write also in complex notation

$$
F(z)=F(x, y)=U+i V, \quad z=x+i y .
$$

It is to be noted that $U$ and $V$ satisfy the equations

$$
\begin{aligned}
V_{x}+U_{y} & =0, \\
\frac{2 \lambda}{y} V+V_{y}-U_{x} & =0, \quad 0 \leqq \lambda .
\end{aligned}
$$

Recall also that if $f(r, \theta)$ is the Poisson integral of a function, say in $L^{p}\left(d m_{\lambda}\right)$, $1<p<\infty$, then by Theorems 2 and 4 it follows that

$$
\sup _{r<1} \int_{0}^{\pi}\left|F\left(r e^{i \theta}\right)\right|^{p} d m_{\lambda}(\theta)<\infty
$$

We are now in a position to make our basic definition. Let $F=U+i V$ be a function regular in the unit disc. $U$ is even in $y$ and $V$ is odd in $y$. Suppose that $F$ satisfies the system (9.3) in the upper semi-disc, and for some $p, p>0, F$ satisfies the boundedness condition (9.4). Then we say that $F$ belongs to the class $H^{p}$.

By the remarks made above it can be shown that the $H^{p}$ theory, when $1<p<\infty$, is essentially equivalent with the $L^{p}$ theory already studied. Our main interest, therefore, will be with $p \leqq 1$. Here matters stand in close analogy with the $H^{p}$ theory of harmonic functions in several variables, developed earlier; see [17]. Thus it will be possible to develop an $H^{p}$ theory for a range of $p$ 's less than 1 , but not all $p$ 's, $0 \leqq p \leqq 1$. More precisely we shall deal with those $p$ 's which satisfy $p \geqq 2 \lambda /(2 \lambda+1)$; for only those we have the following basic lemma concerning "sub-harmonicity." 
Lemma 5. Let $F=U+i V$ satisfy (9.3) in a region in the upper half-plane. Suppose $|F|>0$ there. Then if $p \geqq 2 \lambda /(2 \lambda+1)$, and $\Delta_{\lambda}=\partial^{2} / \partial x^{2}+\partial^{2} / \partial y^{2}$ $+(2 \lambda / y) \partial / \partial y$ (where $\lambda \geqq 0)$, then

$$
\Delta_{\lambda}|F|^{p} \geqq 0 .
$$

Proof. Write $F=(U, V), F_{x}=\left(U_{x}, U_{x}\right)$ and $F \cdot F_{x}=U U_{x}+V V_{x} \cdots$, etc. Then

$$
\begin{aligned}
& \frac{\partial}{\partial x}|F|^{p}=p|F|^{p-2} F_{x} \cdot F, \\
& \frac{\partial}{\partial y}|F|^{p}=p|F|^{p-2} F_{y} \cdot F, \\
& \frac{\partial^{2}}{\partial x^{2}}|F|^{p}=p\left[(p-2)|F|^{p-4}\left(F_{x} \cdot F\right)^{2}+|F|^{p-2}\left\{\left|F_{x}\right|^{2}+F \cdot F_{x x}\right\}\right] .
\end{aligned}
$$

Similarly with $\left(\partial^{2} / \partial y^{2}\right)|F|^{p}$.

Adding we obtain

$$
\begin{aligned}
& \Delta_{\lambda}|F|^{p} \\
& =p|F|^{p-4}\left[(p-2)\left(\left(F_{x} \cdot F\right)^{2}+\left(F_{y} \cdot F\right)^{2}\right)+|F|^{2}\left\{\left|F_{x}\right|^{2}+\left|F_{y}\right|^{2}+F \cdot \Delta_{\lambda} F\right\}\right] .
\end{aligned}
$$

Notice, however, that $\Delta_{\lambda} F=\Delta_{\lambda} U+i \Delta_{\lambda} V$. From (9.3) it follows that $\Delta_{\lambda} U=0$, while

$$
V_{x x}+V_{y y}+\left(\frac{2 \lambda}{y} V\right)_{y}=0
$$

thus $\Delta_{\lambda} V=\left(2 \lambda / y^{2}\right) V$, and hence $F \cdot \Delta_{\lambda} F=2 \lambda\left(V^{2} / y^{2}\right)$. Substituting in (9.6) we then see that $\Delta_{\lambda}|F|^{p} \geqq 0$ if $p \geqq 2$, since everything is positive in this case. Having disposed of this simpler case, let us now suppose that $p<2$. Then (9.5) is equivalent with

$$
\left(F_{x} \cdot F\right)^{2}+\left(F_{y} \cdot F\right)^{2} \leqq \frac{1}{2-p}|F|^{2}\left[\left|F_{x}\right|^{2}+\left|F_{y}\right|^{2}+\frac{2 \lambda V^{2}}{y^{2}}\right] .
$$

Let $M$ denote the matrix

$$
\left[\begin{array}{rr}
U_{x} & V_{x} \\
-U_{y} & -V_{y}
\end{array}\right]
$$

Notice that the equations (9.3) can be translated into the two statements(i) $M$ is symmetric and (ii) trace of $M=2 \lambda V / y$. The inequality (9.7) can be translated to

$$
|M[F]|^{2} \leqq \frac{1}{2-p}|F|^{2}\left[\|M\|^{2}+\frac{(\operatorname{tr}(M))^{2}}{2 \lambda}\right],
$$

where $\|M\|^{2}$ denotes the square of the 'Hilbert-Schmidt' norm of the matrix. 
If we consider $F$ to be an arbitrary two-component vector, then (9.8) becomes

$$
|M|^{2} \leqq\left(\frac{1}{2-p}\right)\left[\|M\|^{2}+\frac{(\operatorname{tr}(M))^{2}}{2 \lambda}\right],
$$

where $|M|$ denotes the usual norm of the matrix $M-$ considered as an operator. We shall show that the inequality holds for any symmetric $2 \times 2$ matrix $M$, as long as $p \geqq 2 \lambda /(2 \lambda+1)$.

In fact, $|M|,\|M\|$, and $\operatorname{tr}(M)$ are invariant under orthogonal changes of coordinates; so we may assume that $M$ is already in diagonal form,

$$
M=\left(\begin{array}{cc}
\mu_{1} & 0 \\
0 & \mu_{2}
\end{array}\right)
$$

Then (9.9) becomes

$$
\max _{k} \mu_{k}^{2} \leqq \frac{1}{(2-p)}\left[\mu_{1}^{2}+\mu_{2}^{2}+(2 \lambda)^{-1}\left(\mu_{1}+\mu_{2}\right)^{2}\right]
$$

We have

$$
\mu_{1}=-\mu_{2}+\left(\mu_{1}+\mu_{2}\right) \text {. }
$$

Therefore, $\mu_{1}^{2} \leqq \alpha \mu_{2}^{2}+\beta\left(\mu_{1}+\mu_{2}\right)^{2}$, whenever $\alpha>0, \beta>0,1 / \alpha+1 / \beta=1$. Add $\alpha \mu_{1}^{2}$ to both sides; this gives

$$
\mu_{1}^{2} \leqq \frac{\alpha}{1+\alpha}\left(\left[\mu_{1}^{2}+\mu_{2}^{2}\right]+\frac{\beta}{\alpha}\left[\mu_{1}+\mu_{2}\right]^{2}\right) .
$$

Choose $\beta=(2 \lambda+1) / 2 \lambda, \quad \alpha=2 \lambda+1$; then $\beta / \alpha=1 / 2 \lambda$, and $\alpha /(1+\alpha)$ $=(2 \lambda+1) /(2 \lambda+2)$. Thus $(9.10)$ is valid as long as $1 /(2-p) \geqq(2 \lambda+1) /(2 \lambda+2)$, which is $2>p \geqq 2 \lambda /(2 \lambda+1)$. This proves (9.10) and, therefore, the lemma.

REMARK. The lower bound $2 \lambda /(2 \lambda+1)$ which occurs for $p$ in Lemma 5 cannot be improved. One only need consider $U=x\left(x^{2}+y^{2}\right)^{-\lambda-1}$ and $V=-y\left(x^{2}+y^{2}\right)^{-\lambda-1}$. Then $|F|^{p}=\left(r^{-2 \lambda-1}\right)^{p}=r^{-\mu}$. But $\Delta_{\lambda} r^{-\mu}<0$ unless $\mu$ $\geqq 2 \lambda$.

We now come to the majorization theorem which is the key result in the development of the $H^{p}$ results.

Theorem 5. Let $\lambda>0$, and $v=2 \lambda /(2 \lambda+1)$. Suppose that $F \in H^{p}, v \leqq p$. (a) If $p=v$, there exists finite positive measure $d \mu(d \mu \in B)$, so that if $h(r, \theta)$ is its Poisson integral, then

$$
\left|F\left(r e^{i \theta}\right)\right|^{v} \leqq h(r, \theta), \quad 0 \leqq r<1, \quad \text { for all } \theta,
$$

and

$$
\sup _{r<1} \int_{0}^{\pi}\left|F\left(r e^{i \theta}\right)\right|^{\nu} d m_{\lambda}(\theta)=\int_{0}^{\pi} \sin ^{2 \lambda} \theta d \mu(\theta)(=\|d \mu\|) .
$$


(b) If $p>v$, then there exists a function $g(\theta) \in L^{q}$ where $q=p / v$, so that if $h(r, \theta)$ is the Poisson integral of $g$, then (9.11) still holds. (9.12) is then to be replaced by

$$
\sup _{r<1}\left\|F\left(r e^{i \theta}\right)\right\|_{p}^{v}=\|g\|_{q} .
$$

Proof. Let us consider first part (a). Fix $\rho, 0<\rho<1$, and set $g_{\rho}(\theta)=\left|F\left(\rho e^{i \theta}\right)\right|^{v}$. Let $h_{\rho}(r, \theta)$ be the Poisson integral of $g_{\rho}(\theta)$. The main step in the proof consists in proving the inequality

$$
\left|F\left(\rho r e^{i \theta}\right)\right| \leqq h_{\rho}(r, \theta), \quad 0 \leqq r \leqq 1 .
$$

For this purpose consider the difference $\delta(r, \theta)=\left|F\left(\rho r e^{i \theta}\right)\right|-h_{\rho}(r, \theta)$. We observe that $h_{\rho}(r, \theta)$ is regular, and even in the unit disc and satisfies $\Delta_{\lambda}\left(h_{\rho}\right)=0$ there, since $h_{\rho}$ is a Poisson integral. Moreover, recalling the properties of $U, V$ and $F$, we see that $\left|F\left(\rho r e^{i \theta}\right)\right|$ is even and regular near any point where $|F|>0$, and satisfies $\Delta_{\lambda}|F| \geqq 0$, there, by Lemma 5 . Notice also that $\delta(1, \theta)=0$, by the definition of $h_{\rho}$. Suppose now, that contrary to (9.13) we had $\delta(r, \theta)>0$ for some point $(r, \theta)$. Since $\delta$ is continuous in the closed disc and vanishes on the boundary, its maximum would be attained at some point $\left(r_{0}, \theta_{0}\right)$ and $\delta\left(r_{0}, \theta_{0}\right)>0$. However $h(r, \theta) \geqq 0$, since it is a Poisson integral of a non-negative function. Therefore $\left|F\left(\rho r_{0} e^{i \theta}\right)\right|>0$, and hence $\Delta_{\lambda}(\delta) \geqq 0$ near the point $\left(r_{0}, \theta_{0}\right)$. This would violate the maximum principle (Theorem 1 of $\S 3$ ), and hence (9.13) is proved.

Now let $\rho_{n}$ be a sequence tending to 1 from below. Let $g_{n}(\theta)=\left|F\left(\rho_{n} e^{i \theta}\right)\right|$ and let $h_{n}(r, \theta)$ be the Poisson integral of $g_{n}(\theta)$. Then by (9.13) we have

$$
\left|F\left(\rho_{n} r e^{i \theta}\right)\right|^{v} \leqq h_{n}(r, \theta) \text {. }
$$

Moreover,

$$
\int_{0}^{\pi} g_{n}(\theta) \sin ^{2 \lambda} \theta d \theta \leqq \sup _{\rho<1} \int_{0}^{\pi}\left|F\left(\rho e^{i \theta}\right)\right|^{v} \sin ^{2 \lambda} \theta d \theta<\infty .
$$

Thus, as is well known, we can find a subsequence $\left\{g_{n_{k}}(\theta)\right\}$ and a measure $d \mu$, so that, $g_{n_{k}} \rightarrow d \mu$ weakly. Let $h(r, \theta)$ be the Poisson integral of $d \mu$. From (9.14) it follows that

$$
\left|F\left(r e^{i \theta}\right)\right| \leqq h(r, \theta), \quad 0 \leqq r<1,
$$

and from (9.15) that

$$
\begin{aligned}
\int_{0}^{\pi} \sin ^{2 \lambda}(\theta) d \mu(\theta) & \leqq \sup _{r<1} \int_{0}^{\pi}\left|F\left(r e^{i \theta}\right)\right|^{v} \sin ^{2 \lambda} \theta d \theta . \\
\int_{0}^{\pi} h(r, \theta) \sin ^{2 \lambda} \theta d \theta & =\int_{0}^{\pi} \sin ^{2 \lambda}(\theta) d \mu(\theta) .
\end{aligned}
$$


Combining this with (9.16) and (9.17) gives the identity (9.12). Thus part (a) of the theorem is completely proved. Part (b) is proved in a very similar way and needs no further comment.

10. Theory of $H^{p}$-space: Basic results. We are now in a position to state the basic results regarding the theory of $H^{p}$-spaces for ultraspherical expansions. The proofs given below depend on two ideas: the theory of majorization developed in the preceding section, which gives certain global results, and the "similarity principle" for pseudo-analytic functions, which will lead to certain local (i.e., almost everywhere) conclusions.

Let us recall the definition of the $H^{p}$-spaces given at the beginning of $\S 9$. We then have

THEOREM 6. (a) Let $F \in H^{p}, 2 \lambda /(2 \lambda+1) \leqq p$. Then $\lim _{r \rightarrow 1} F\left(r e^{i \theta}\right)=F\left(e^{i \theta}\right)$ exists for almost every $\theta$. More generally, for almost every $\theta, \lim F\left(\rho e^{i \phi}\right)$ exists as $\rho e^{i \phi}$ approaches the point $e^{i \theta}$ nontangentially.

(b) If $F\left(e^{i \theta}\right)=0$ in a set of $\theta$ of positive measure, then $F \equiv 0$.

(c) If $2 \lambda /(2 \lambda+1)<p$, then $\int_{0}^{\pi}\left|F\left(r e^{i \theta}\right)-F\left(e^{i \theta}\right)\right|^{p} d m_{\lambda}(\theta) \rightarrow 0$, as $r \rightarrow 1$.

As a simple consequence of part (c) of the theorem, when $p=1$, we obtain the following analogue of the theorem of F. and M. Riesz.

Corollary 1. Let $d \mu_{1}$ and $d \mu_{2}$ be two finite measures (that is, $\left.\int_{0}^{\pi}(\sin \theta)^{2 \lambda}\left|d \mu_{i}\right|<\infty\right)$. Suppose that

$$
d \mu_{1} \sim \sum a_{n} P_{n}^{\lambda}(\cos \theta)
$$

and

$$
d \mu_{2} \sim 2 \lambda \sum \frac{a_{n}}{n+2 \lambda} \sin P_{n-1}^{\lambda+1}(\cos \theta) .
$$

Then both $d \mu_{1}$ and $d \mu_{2}$ are absolutely continuous.

We also can complete the generalization of Fatou's theorem given Corollary 2 of Theorem 3, $\$ 6$.

COROLlary 2. Let $u(x, y)$ be $\lambda$-harmonic, regular and even and satisfy

$$
\sup _{0 \leqq r<1} \int_{0}^{\pi}|u(r \cos , r \sin \theta)| d m_{\lambda}(\theta)<\infty .
$$

Then $\lim _{r \rightarrow 1} u(r \cos \theta, r \sin \theta)$ exists for a.e. $\theta$, and in fact nontangentially.

We come now to the proof of the theorem and its corollaries. Let $d \mu$ be a positive measure which is finite, i.e., $\int_{0}^{\pi} \sin ^{2 \lambda} \theta d \mu(\theta)<\infty$. Let $h(r, \theta)$ denote its Poisson integral

$$
h(r, \theta)=\int_{0}^{\pi} P(r, \theta, \phi) \sin ^{2 \lambda} \phi d \mu(\phi) .
$$


We denote by $\mu^{*}(\theta)$ the maximal function associated to $d \mu$, that is

$$
\mu^{*}(\theta)=\sup _{h} \frac{\int_{\theta}^{\theta+h} \sin ^{2 \lambda} \phi d \mu(\phi)}{\int_{\theta}^{\theta+1} \sin ^{2 \lambda} \phi d \phi}
$$

The well-known theorem of differentiation of measures tells us that $\mu^{*}(\theta)<\infty$ for a.e. $\theta$.

Let us observe that

$$
\sup _{0 \leqq r<1} h(r, \theta) \leqq c \mu^{*}(\theta) .
$$

In fact, in the case when $d \mu$ is absolutely continuous (10.1) is merely a restatement of Lemma 3 of $\$ 5$. The proof for a not-necessarily absolutely continuous measure is exactly the same.

(10.1) gives us an appropriate estimate for the radial approach to the boundary. In what follows we shall need "a nontangential" analogue of (10.1) This may be formulated as follows. Let $\Omega$ be a fixed triangle, lying strictly inside the unit disc, except that one of its vertices is at the point $r=1, \theta=0$. For any $\theta_{0}$, define $\Omega\left(\theta_{0}\right)$ to be the triangle rotated (about the origin) so that this vertex is the point $r=1, \theta=\theta_{0}$. The chosen triangle $\Omega=\Omega(0)$ is kept fixed in the rest of our discussion. Its particular shape is irrelevant.

We can then state

LEMMA 6.

$$
\sup _{r e^{i \psi} \in \Omega(\theta)} h\left(r, e^{i \psi}\right) \leqq c \mu^{*}(\theta) .
$$

Proof. In view of the definition of the Poisson kernel quoted above, it suffices to show that

$$
P(r, \psi, \phi) \leqq c P(r, \theta, \phi), \quad r e^{i \psi} \in \Omega(\theta) .
$$

Let us recall, however, that

where

$$
P(r, \theta, \phi)=\left(\frac{\lambda}{\pi}\right)\left(1-r^{2}\right) \int_{0}^{\pi} \frac{\sin ^{2 \lambda-1} t}{D^{\lambda+1}} d t,
$$

$$
\begin{aligned}
D & =1-2 r(\cos \theta \cos \phi+\sin \theta \sin \phi \cos t)+r^{2} \\
& =(x-\xi)^{2}+(y-\eta)^{2}+2 y \eta[1-\cos t],
\end{aligned}
$$

where $(\xi, \eta)=(\cos \phi, \sin \phi)$ and $(x, y)=(r \cos \theta, r \sin \theta)$, (see (2.12)).

Now the expression $D$ has an interpretation in terms of a distance measured in three-dimensional Euclidean space. Thus consider the $(x y)$-plane embedded 
in three-dimensional Euclidean space, and let $\Delta$ denote the disc perpendicular to the (xy)-plane and be determined by the fact that $(\xi, \eta, 0)$ and $(\xi,-\eta, 0)$ are a pair of diametrically opposite points on its perimeter. Then if $P=(x, y, 0)$ and $Q$ is an appropriate point in the perimeter of this disc, then $D$ is the square of the distance from $P$ to $Q$. The point $Q$ may be fixed on the perimeter of $\Delta$ as follows: $t$ is the angle made by the ray joining $(\xi, 0,0)$ and $Q$ with the $y$ direction.

From this it can be seen that

$$
D(r, \theta, \phi, t) \leqq c D(r, \psi, \phi, t), \quad r e^{i \psi} \in \Omega(\theta),
$$

and therefore (10.2) follows. This proves the lemma.

We revert now to the proof of Theorem 6 and consider first part (a). Let $\delta>0$, and let $R_{\delta}$ be the open region consisting of that part of the open unit disc where $y>\delta$, and let $\bar{R}_{\delta}$ denote its closure. Restrict $F(z)=U(x, y)+i V(x, y)$ to $R_{\delta}$. Then $F$ satisfies the system of "Cauchy-Riemann" equation (9.3) where the coefficients are regular in $\bar{R}_{\delta}$. Therefore by the similarity principle for pseudoanalytic functions (see, e.g., Bers [2]) there exists a function $S(z)$ continuous in $\bar{R}_{\delta}$, and an analytic function $G(z)$ in $R_{\delta}$, so that

$$
F(z)=e^{S(z)} G(z), \quad z \in R_{\delta} .
$$

However, by the majorization theorem (Theorem 5$),|F(z)|^{v} \leqq h(r, \theta)$, where $h(r, \theta)$ is the Poisson integral of a finite positive measure $d \mu(\theta)$. Thus in view of Lemma $6, F\left(r e^{i \theta}\right)$ is bounded for $r e^{i \theta} \in \Omega\left(\theta_{0}\right)$ and for almost every $\theta_{0}$. By (10.3) $G\left(r e^{i \theta}\right)$ is bounded in the same triangles, for almost all $\theta_{0}$, so that $\sin \theta_{0} \geqq \delta$. Then by the theorem of Privalov-Plessener (see [26, Vol. II, p. 200]), it follows that $G(z)$ has nontangential limits on the perimeter of unit disc for a.e. $\theta_{0}$, so that $\sin \theta_{0} \geqq \delta>0$. By (10.3) (since $S(z)$ is continuous in $\vec{R}_{\delta}$ ), the same is true for $F(z)$. Finally, since $\delta>0$, but is otherwise arbitrary, part (a) of the theorem is completely proved. Part (b) is proved similarly, using the fact that $G$ can vanish only on a set of zero measure on the perimeter of the disc unless it vanishes identically.

We come now to the proof of part (c).

Let $p>v=2 \lambda /(\lambda+1)$, and $q=p / v$. Then by part (b) of Theorem 5, there exists a $g \in L^{q}$, so that if $h(r, \theta)$ is its Poisson integral

$$
\left|F\left(r e^{i \theta}\right)\right|^{v} \leqq h(r, \theta) .
$$

However, by Lemmas 2 and 3 ,

Therefore

$$
\sup _{r<1} h(r, \theta) \leqq c g^{*}(\theta) \in L^{q} .
$$

$$
\left|F\left(r e^{i \theta}\right)-F\left(e^{i \theta}\right)\right|^{p} \leqq c g^{*}(\theta),
$$


and part (c) of the theorem is proved by the use of the Lebesgue dominated convergence theorem, in conjunction with part (a), which is already known.

We now come to the proof of Corollary 1. Suppose that $d \mu_{1} \sim \sum a_{n} P_{n}^{\lambda}(\cos \theta)$ and $d \mu_{2} \sim 2 \lambda \sum\left(a_{n} /(n+2 \lambda)\right) P_{n-1}^{\lambda+1}(\cos \theta)$.

Let

$$
\begin{aligned}
& U(x, y)=\sum a_{n} r^{n} P_{n}^{\lambda}(\cos \theta), \quad r<1, \quad(x, y)=(r \cos \theta, r \sin \theta), \\
& V(x, y)=2 \lambda \sum \frac{a_{n}}{n+2 \lambda} r^{n} \sin \theta P_{n-1}^{\lambda+1}(\cos \theta) .
\end{aligned}
$$

Then, as we know, $F=U+i V$ satisfies the system (9.3), and because $U$ and $V$ are Poisson integrals, then

$$
\int_{0}^{\pi}\left|F\left(r e^{i \theta}\right)\right| d m_{\lambda}(\theta) \leqq\left\|d \mu_{1}\right\|+\left\|d \mu_{2}\right\|
$$

Thus $F$ is in $H^{1}$, and therefore there exists $F\left(e^{i \theta}\right)=f(\theta)+i f(\theta) \in L^{1}\left(d m_{\lambda}\right)$, so that

$$
\int_{0}^{\pi}\left|F\left(r e^{i \theta}\right)-F\left(e^{i \theta}\right)\right| d m_{\lambda}(\theta) \rightarrow 0
$$

Then it is easily verified that $f(\theta) d \theta=d \mu_{1}(\theta)$ and $\tilde{f}(\theta) d \theta=d \mu_{2}(\theta)$, since both $f(\theta) d \theta$ and $d \mu_{1}$ have the same ultraspherical expansions of index $\lambda$, and $\tilde{f}(\theta) d \theta$, $d \mu_{2}$ have the same expansions of index $\lambda+1$.

To prove Corollary 2 recall that the assumptions on $u$ imply that it is the Poisson integral of a finite measure. (See Theorem $3^{\prime}$ in \$6.) If we now define $F(z)=U(x, y)+i V(x, y)$ as above it then follows by Theorem 4, Corollary 2 that $F \in H^{p}$, for all $p<1$. The result then follows from part (a) of the present theorem.

We should point out conclusion (a) and (b) of the theorem could be proved for all $p, 0<p$, without the extra restriction $2 \lambda /(2 \lambda+1) \leqq p$. This could be done as follows. Define, as in the proof of the theorem, the function $G(z)$, analytic in $R_{\sigma}$, so that (10.3) holds. Then $\sup _{r<1} \int_{0}^{\pi}\left|F\left(r e^{i \theta}\right)\right|^{p} d m_{\lambda}(\theta)<\infty$ implies easily $\sup _{r<1, \sin \theta \geqq \delta} \int\left|G\left(r e^{i \theta}\right)\right|^{p} d \theta<\infty$.

Then a modification of the classical arguments shows that $G$ has nontangential limits for almost all points on the boundary of the disc. The same then follows for $F(z)$. Similar arguments hold for the nonvanishing on the boundary.

\section{ChAPTER II}

11. Partial sums of ultraspherical expansions. The purpose of the next few sections is to develop an analogue of the Littlewood-Paley theory for ultraspherical 
expansions, culminating in the "multiplier theorem" of $\$ 14$.

In the trigonometric case there are two basic tools used in the development of the Littlewood-Paley theory. The first are certain inequalities for partial sums, and the second deals with the auxiliary " $g$-function." In this section we shall extend the partial sum inequalities to the ultraspherical case.

Thus let

$$
f(\theta) \sim \sum a_{n} P_{n}^{\lambda}(\cos \theta)
$$

and define $S_{n}$ by

$$
S_{n}(f)=S_{n}(\theta ; f)=\sum_{k=0}^{n} a_{k} P_{k}^{\lambda}(\cos \theta) .
$$

A basic result for the partial sums is the inequality

$$
\left\|S_{n}(f)\right\|_{p} \leqq A_{p}\|f\|_{p}, \quad \frac{2 \lambda+1}{\lambda+1}<p<\frac{2 \lambda+1}{\lambda} .
$$

The case corresponding to $\lambda=0$ is of course the classical inequality of $\mathbf{M}$. Riesz, and the case for positive $\lambda$ is due to Pollard [14].

We shall need an extension of (11.1) which can be stated as follows:

Let $f_{1}, f_{2}, \cdots, f_{k}$ be a given $k$-tuple of functions ( $k$ arbitrary) and write $F=\left(f_{1}, \cdots, f_{k}\right),|F|=\left(\left|f_{1}\right|^{2}+\left|f_{2}\right|^{2}+\cdots+\left|f_{k}\right|^{2}\right)^{1 / 2}$.

Similarly let $n_{1}, \cdots, n_{k}$ be an arbitrary $k$-tuple of positive integers, and write $S(F)$ for the $k$-tuple

$$
S(F)=\left(S_{n_{1}}\left(f_{1}\right), S_{n_{2}}\left(f_{2}\right), \cdots, S_{n_{k}}\left(f_{k}\right)\right) .
$$

Using this notation our result can then be stated as

THEOREM 7.

$$
\||S(F)|\|_{p} \leqq A_{p}\||F|\|_{p}, \quad \frac{2 \lambda+1}{\lambda+1}<p<\frac{2 \lambda+1}{\lambda}
$$

with $A_{p}$ independent of $k$.

The proof of the theorem makes use of the basic reduction of Pollard which occurs in the proof of (11.1) together with the following known lemma, see [26, Vol. II, p. 224].

LeMma 7. Let $T$ be a linear transformation which satisfies

$$
\|T f\|_{p} \leqq A\|f\|_{p} \text { for some } p .
$$

Write $|T(F)|=\left(\left|T f_{1}\right|^{2}+\left|T f_{2}\right|^{2}+\cdots+\left|T f_{k}\right|^{2}\right)^{1 / 2}$, with $|F|=\left(\left|f_{1}\right|^{2}+\cdots+\left|f_{k}\right|^{2}\right)^{1 / 2}$. Then $\||T(F)|\|_{p} \leqq A\||F|\|_{p}$. A is the same constant as in (11.2). 
One should observe the difference between this lemma and Theorem 7 . In the lemma we have one fixed operator, $T$, acting simultaneously on a $k$-tuple of functions. In the theorem we deal with $k$ different partial sums, acting on the $k$-tuple of functions.

We now come to the proof of the theorem, which we have indicated is based on Pollard's work. Thus to avoid duplication we shall repeat only the main steps of his reduction omitting details. Following his notation, $x$ and $y$ are variables in the interval $(-1,1)$, which are related by our variables by the change $x=\cos \theta, y=\cos \phi$.

We have $\omega(x) d x=(\sin \theta)^{2 \lambda} d \theta$ as the basic measure with $\omega(x)=\left(1-x^{2}\right)^{\lambda-1 / 2}$. We write $p_{n}(x)=\gamma_{n}^{1 / 2} P_{n}^{\lambda}(\cos \theta)$, which are the normalized polynomials for the weight $\omega(x)$. $\left(\gamma_{n}\right.$ are the factors given in (2.9).) We also have the conjugate polynomials $q_{n}(x)$, defined by $\left(1-x^{2}\right)^{-1} q_{n}(x)=\left(\gamma_{n}^{*}\right)^{1 / 2} P_{n-1}^{\lambda+1}(\cos \theta)$, which are the orthonormal polynomials with respect to $\left(1-x^{2}\right) \omega(x)$. (See Pollard [14, p. 356].)

Two basic estimates satisfied by the $p_{n}$ and $q_{n}$ are

$$
\begin{aligned}
\left|\left(1-x^{2}\right)^{1 / 4} \omega(x)^{1 / 2} p_{n}(x)\right| & \leqq A, \\
\left|\left(1-x^{2}\right)^{-1 / 4} \omega(x)^{1 / 2} q_{n}(x)\right| & \leqq A .
\end{aligned}
$$

(See Pollard [14, p. 362]; also Szegö [18, 7.33.6, p. 167].)

Now consider the Dirichlet kernel $k_{n}(x, y)$ given by

$$
k_{n}(x, y)=\sum_{j=0}^{n} p_{j}(x) p_{j}(y) \text {. }
$$

According to Christoffel's formula (see Szegö [18, p. 42]),

for appropriate constants $c_{n}$.

$$
k_{n}(x, y)=c_{n} \frac{p_{n+1}(x) p_{n}(y)-p_{n}(x) p_{n+1}(y)}{x-y}
$$

However, the $q_{n}$ 's can be expressed in terms of the $p_{n}$ 's (see Pollard [14, p. 357]). Making the substitution and combining terms gives

$$
k_{n}(x, y)=\alpha_{n} \frac{p_{n+1}(x) q_{n}(y)}{x-y}+\beta_{n} \frac{q_{n}(x) p_{n+1}(y)}{x-y}+\delta_{n} p_{n+1}(x) p_{n+1}(y),
$$

where $\left\{\alpha_{n}\right\},\left\{\beta_{n}\right\}$, and $\left\{\delta_{n}\right\}$ are bounded sequences. (See $[14$, p. 358].)

We quote also the following lemma of Pollard:

LEMMA 8. Let $(T f)(x)=\int_{-1}^{1} K(x, y) f(y) d y$, where

$$
K(x, y)=\left|\frac{\left(\frac{1-y^{2}}{1-x^{2}}\right)^{c}-1}{x-y}\right|,
$$

with $-1<c<1$ and $c<1 / p<c+1,1<p$. Then 


$$
\int_{-1}^{1}|T f(x)|^{p} d x \leqq A^{p} \int_{-1}^{1}|f(x)|^{p} d x .
$$

Now

$$
S_{n}(f)=\int_{-1}^{1} k_{n}(x, y) f(y) \omega(y) d y=S_{n}^{1}(f)+S_{n}^{2}(f)+S_{n}^{3}(f) .
$$

Here $S_{n}^{1}$ is the transformation whose kernel is $\alpha_{n} p_{n+1}(x) q_{n}(y) /(x-y) ; S_{n}^{2}$ has the kernel $\beta_{n} q_{n}(x) p_{n+1}(y) /(x-y)$ and $S_{n}^{3}$ has the kernel $\gamma_{n} p_{n+1}(x) p_{n+1}(y)$.

Let $f_{1}, \cdots, f_{k}$ be $k$ given functions and $n_{1}, \cdots, n_{k}$ be given positive integers. For $1 \leqq j \leqq 3$, set

and

$$
S^{(j)}(F)=\left(S_{n_{1}}^{j}\left(f_{1}\right), \cdots, S_{n_{k}}^{j}\left(f_{k}\right)\right)
$$

$$
\left|S^{j}(F)\right|=\left(\left|S_{n_{1}}^{j}\left(f_{1}\right)\right|^{2}+\cdots+\left(\left|S_{n_{k}}^{j}\left(f_{k}\right)\right|^{2}\right)^{1 / 2}\right.
$$

It will then suffice to show

$$
\int_{-1}^{1}\left|S^{j}(F)(x)\right|^{p} \omega(x) d x \leqq A_{p}^{p} \int_{-1}^{1}|F(x)|^{p} \omega(x) d x, j=1,2,3 .
$$

Consider $S^{1}(F)$ first.

In view of the inequalities (11.3) and the boundedness of $\alpha_{n}$ we can write

$$
\begin{aligned}
& \omega^{1 / p}(x) S_{n_{k}}^{1}\left(f_{k}\right)(x) \\
& =B_{n_{k}}(x) \int_{-1}^{1} \frac{\omega^{1 / p}(y) f_{k}(y) A_{n_{k}}(y) d y}{x-y}+C_{n_{k}}(x) \int_{-1}^{1} K(x, y) \omega^{1 / p}(y) f_{k}(y) A_{n_{k}}(y) d y,
\end{aligned}
$$

where $A_{n}(x), B_{n}(x)$, and $C_{n}(x)$ are uniformly bounded and $K(x, y)$ is the kernel of Lemma 8 , with $c=1 / 4+(\lambda-1 / 2)(1 / 2-1 / p)$.

Apply now Lemmas 7 and 8 , and the well-known fact that

$$
\int_{-1}^{1}\left|\int_{-1}^{1} \frac{f(y) d y}{x-y}\right|^{p} d x \leqq A_{p}^{p} \int_{-1}^{1}|f(x)|^{p} d x .
$$

This gives (11.5) for $j=1$,

$$
\frac{2 \lambda+1}{\lambda+1}<p<\frac{2 \lambda+1}{\lambda} \text {. }
$$

A very similar argument holds for $S^{2}$.

To consider $S^{3}$ we recall that $\left|p_{n+1}(x)\right| \leqq A\left(1-x^{2}\right)^{-\lambda / 2}$ (see (11.3)). Hence $\left|S_{n_{k}}\left(f_{k}\right)\right| \leqq A\left(1-x^{2}\right)^{-\lambda / 2} \int_{-1}^{1} f(y)\left(1-y^{2}\right)^{-\lambda / 2} \omega(y) d y\left(\omega(y)=\left(1-y^{2}\right)^{\lambda-1 / 2}\right)$. 
But the transformation $T$

$$
T: f(x) \rightarrow\left(1-x^{2}\right)^{-\lambda / 2} \int_{-1}^{1} f(y)\left(1-y^{2}\right)^{-\lambda / 2}\left(1-y^{2}\right)^{\lambda-1 / 2} d y
$$

satisfies

$$
\int_{-1}^{1}|T f(x)|^{p}\left(1-x^{2}\right)^{\lambda-1 / 2} d x \leqq A_{p} \int_{-1}^{1}|f(x)|^{p}\left(1-x^{2}\right)^{\lambda-1 / 2} d x
$$

(by Hölder's inequality) when $(2 \lambda+1) /(\lambda+1)<p<(2 \lambda+1) / \lambda$. Therefore, another application of Lemma 7 shows (11.5) when $j=3$, and concludes the proof of the theorem.

REMARK. It should be noted that a basic step in the proof is the passage from the Christoffel formula, which involves only the $p_{n}$ 's, to the formula (11.4), which involves in addition the conjugate polynomials $q_{n}$. Here we have another instance of the significance of the notion of conjugacy studied in Chapter I of this paper.

We shall now prove a rather technical corollary to the theorem, which, however, is important for its applications in $\$ 12$.

Besides the $k$-tuple $f_{1}, f_{2}, \cdots, f_{k}$, considered above, we shall also deal with the respective Poisson integrals $f_{1}(r, \theta), f_{2}(r, \theta), \cdots, f_{k}(r, \theta)$. We select an arbitrary $k$-tuple of $r$ 's, $r_{1}, r_{2}, \cdots, r_{k}$, with $0 \leqq r_{j}<1$, and we let $\delta_{j}$ denote an arbitrary sub-interval of $\left(r_{j}, 1\right)$, with $\left|\delta_{j}\right|$ standing for the length of $\delta_{j}$.

COROllary.

$$
\left\|\left(\sum_{j=1}^{k}\left|S_{n_{j}}\left(\theta, f_{j}\left(r_{j}, \theta\right)\right)\right|^{2}\right)^{1 / 2}\right\|_{p}
$$

$$
\leqq A_{p}^{\prime}\left\|\left(\sum_{j=1}^{k}\left|\delta_{j}\right|^{-1} \int_{\delta_{j}}\left|f_{j}(\rho, \theta)\right|^{2} d \rho\right)^{1 / 2}\right\|_{p}, \frac{2 \lambda+1}{\lambda+1}<p<\frac{2 \lambda+1}{\lambda} .
$$

Note. The constant $A_{p}^{\prime}$ may be taken to be $\left(A_{p}\right)^{2}$, where $A_{p}$ is the constant arising in the theorem.

Let us set $F=\left(f_{1}, \cdots, f_{k}\right),|F|=\left(\left|f_{1}\right|^{2}+\cdots+\left|f_{k}\right|^{2}\right)^{1 / 2}$ and $F(r, \theta)=\left(f_{1}\left(r_{1}, \theta\right)\right.$, $\left.f_{2}\left(r_{2}, \theta\right), \cdots, f_{k}\left(r_{k}, \theta\right)\right)$, with

$$
|F(r, \theta)|=\left(\left|f_{1}\left(r_{1}, \theta\right)\right|^{2}+\cdots+\left|f_{k}\left(r_{k}, \theta\right)\right|^{2}\right)^{1 / 2} .
$$

We prove first that if $(2 \lambda+1) /(\lambda+1)<p<(2 \lambda+1) / \lambda$

$$
\||F(r, \theta)|\|_{p} \leqq A_{p}\||F(\theta)|\|_{p} .
$$

In fact

$$
(r, \theta)=\sum_{l=0}^{\infty} a_{l} r^{l} P_{l}(\cos \theta)=(1-r) \sum S_{l}(\theta, f) r^{l}
$$


Therefore,

$$
|F(r, \theta)|^{2}=\sum_{j=1}^{k}\left|f_{j}\left(r_{j}, \theta\right)\right|^{2} \leqq \sum_{j=1}^{k}\left(1-r_{j}\right) \sum_{l=0}^{\infty}\left|S_{l}\left(\theta, f_{j}\right)\right|^{2} r_{j}^{l},
$$

by Schwarz's inequality. Thus if we apply the theorem, we obtain

$$
\begin{aligned}
\||F(r, \theta)|\|_{p} & \leqq A_{p}\left\|\left(\sum_{j=1}^{k}\left(1-r_{j}\right) \sum_{l=0}^{\infty}\left|f_{j}(\theta)\right|^{2} r_{j}^{l}\right)^{1 / 2}\right\|_{p} \\
& =A_{p}\left\|\left(\sum_{j=1}^{k}\left|f_{j}(\theta)\right|^{2}\right)^{1 / 2}\right\|_{p}=A_{p}\||F(\theta)|\|_{p} .
\end{aligned}
$$

Next consider $r_{j}^{\prime}$ restricted by $r_{j}^{\prime}>r_{j}, j=1, \cdots, k$. Define

$$
F\left(r^{\prime}, \theta\right)=\left(f_{1}\left(r_{1}^{\prime}, \theta\right), f_{2}\left(r_{2}^{\prime}, \theta\right), \cdots, f_{k}\left(r_{k}^{\prime}, \theta\right)\right) .
$$

An immediate consequence of (11.8) is the the generalization

$$
\||F(r, \theta)|\|_{p} \leqq A_{p}\left\|\left|F\left(r^{\prime}, \theta\right)\right|\right\|_{p}, \quad r<r^{\prime},
$$

where $r<r^{\prime}$ stands for $r_{j}<r_{j}^{\prime}, j=1, \cdots, k$. (Again $(2 \lambda+1) /(\lambda+1)<p<(2 \lambda+1) / \lambda$.) (11.9) can then be given the following variant. Let $\delta_{j}$ denote a subinterval of $\left(r_{j}, 1\right)$; we have

$$
\||F(r, \theta)|\|_{p}<A_{p}\left\|\left[\sum_{j=1}^{k}\left(1 /\left|\delta_{j}\right|\right) \int_{\delta_{j}}\left|f_{j}(\rho, \theta)\right|^{2} d \rho\right]^{1 / 2}\right\|_{p} .
$$

In fact (11.10) may be proved by breaking the $\delta_{j}$ up into sufficiently small intervals, applying (11.9), and then by passing to the limit.

We now come to the proof of (11.7).

The left side of (11.7) is, by the theorem, bounded by

$$
A_{p}\left\|\left(\sum_{j=1}^{k}\left|f_{j}\left(r_{j}, \theta\right)\right|^{2}\right)^{1 / 2}\right\|_{p}=A_{p}\||F(r, \theta)|\|_{p} .
$$

Apply now (11.10) and we get (11.7) with $A_{p}^{\prime}=\left(A_{p}\right)^{2}$.

12. The $g$-function. We come now to the second auxilliary tool used in the development of the analogue of the Littlewood-Paley theory. The results that we shall obtain are $L^{p}$ inequalities for the $g$-function, which is defined as follows.

Let $f(\theta) \sim \sum a_{n} P_{n}^{\lambda}(\cos \theta)$, and let $f(r, \theta)$ be its Poisson integral,

$$
f(r, \theta)=\sum a_{n} r^{n} P_{n}^{\lambda}(\cos \theta) .
$$

Then we define

$$
g(\theta)=g(\theta ; f)=\left(\int_{0}^{1}(1-r)\left|f_{r}(r, \theta)\right|^{2} d r\right)^{1 / 2}
$$


The usefulness of this function will become clearer in the subsequent section. Suffice it to say that we may regard $g$ as an Abelian analogue of another function which has more immediate application. Our theorem then is

THEOREM 8.

$$
\begin{gathered}
\|g(\theta ; f)\|_{p} \leqq A_{p}\|f\|_{p}, \quad 1<p<\infty . \\
\text { If } \int_{0}^{\infty} f(\theta) \sin ^{2 \lambda} \theta d \theta=0, \text { then }\|f\|_{p} \geqq A_{p}\|g(\theta ; f)\|_{p}, \quad 1<p<\infty .
\end{gathered}
$$

Before we come to the proof of the theorem we shall give an outline of its ideas.

One proves first the direct inequality (12.2), and then the converse (12.3) follows from this by a rather standard duality argument. The main difficulty, therefore, lies in the proof of (12.2). Now

$$
f_{r}(r, \theta)=\int_{0}^{\pi} P_{r}(r, \theta, \phi) f(\phi)(\sin \phi)^{2 \lambda} d \phi .
$$

It is clear that what is needed, among other things, are estimates on the behavior of the differentiated Poisson kernel, $P_{r}(r, \theta, \phi)$. These are given in Lemma 9 below and are in the same spirit as the estimates for the Poisson kernel in Lemma 1 of $\S 4$, and the conjugate kernel in Lemma 4 of $\S 7$.

Let us recall the abbreviations used earlier

$$
\Delta=1-2 r \cos (\theta-\phi)+r^{2} \text { and } \sigma=r \sin \theta \sin \phi .
$$

Then our estimates for $P_{r}(r, \theta, \phi)$ become, if $1 / 2<r<1$,

$$
\begin{aligned}
\left|P_{r}(r, \theta, \phi)\right| & \leqq C / \Delta^{\lambda+1}, \\
P_{r}(r, \theta, \phi) & =P_{r}^{+}(r, \theta, \phi)+P_{r}^{+}(r, \theta,-\phi),
\end{aligned}
$$

where

$$
\begin{aligned}
P_{r}^{+}(r, \theta, \phi)= & \frac{\sigma^{-\lambda}}{\pi} \frac{\partial}{\partial r}\left(\frac{1-r^{2}}{\Delta}\right)+O\left(\frac{\sigma^{-\lambda}(1-r)}{\Delta}\right) \\
& +O\left[\sigma^{-\lambda} /(\sigma+\Delta)\left(1+\log ^{+}(\sigma / \Delta)\right)\right]
\end{aligned}
$$

It is to be noted that the main term of the asymptotic estimate (12.5),

$$
\frac{\sigma^{-\lambda}}{\pi} \frac{\partial}{\partial r}\left(\frac{1-r^{2}}{\Delta}\right),
$$

is, except for the factor $\sigma^{-\lambda}$, essentially the $P_{r}(r, \theta, \phi)$ in the trigonometric case $(\lambda=0)$. The asymptotic formula (12.5) will be used when $\theta$ is near $\phi$; and its main contribution, due to the first theorem, will then be handled by using the classical result for the $g$-function. 
When $\theta$ is not near $\phi$, the simpler estimate (12.4) will be sufficient.

LEMMA 9. The estimates (12.4) and (12.5) hold for $P_{r}(r, \theta, \phi)$ when $1 / 2 \leqq r<1\left(^{6}\right)$.

Proof of lemma. Recall that

$$
P(r, \theta, \phi)=(\lambda / \pi)\left(1-r^{2}\right) \int_{0}^{\pi} \frac{\sin ^{2 \lambda-1} t}{D^{\lambda+1}} d t,
$$

where

$$
D=1-2 r(\cos \theta \cos \phi+\sin \theta \sin \phi \cos t)+r^{2}
$$

We notice two useful facts about $D$

$$
D \geqq \Delta \quad\left(\Delta=1-2 r \cos (\theta-\phi)+r^{2}\right) .
$$

and

$$
(1-r)\left|D_{r}\right| \leqq \frac{2}{r} D
$$

In fact $r D_{r}-r^{2}+1=D$, and $D \geqq(1-r)^{2}$, as can be seen by rewriting $D=(1-r)^{2}+2 r[1-\cos (\theta-\phi)]+2 r \sin \theta \sin \phi[1-\cos t]$. Combining these two gives (12.7).

Differentiating in the integral formula for $P(r, \theta, \phi)$ then gives

$$
\left|P_{r}(r, \theta, \phi)\right| \leqq \frac{\lambda 2 r}{\pi} \int_{0}^{\pi} \frac{\sin ^{2 \lambda-1}}{D^{\lambda+1}} t d t+\frac{\lambda(\lambda+1)}{\pi}\left(1-r^{2}\right) \int_{0}^{\pi} \frac{\left|D_{r}\right|}{D^{\lambda+2}} \sin ^{2 \lambda-1} t d t .
$$

When we substitute the estimates (12.6) and (12.7) (taking into account $1 / 2 \leqq r<1)$, we obtain the first conclusion of the lemma, i.e., (12.4).

We write

$$
P(r, \theta, \phi)=\frac{\lambda\left(1-r^{2}\right)}{\pi} \int_{0}^{\pi} \frac{\sin ^{2 \lambda-1} t}{D^{\lambda+1}} d t=\frac{\lambda\left(1-r^{2}\right)}{\pi} \int_{0}^{\pi / 2}+\frac{\lambda\left(1-r^{2}\right)}{\pi} \int_{\pi / 2}^{\pi} .
$$

Call

$$
P^{+}(r, \theta, \phi)=\frac{\lambda\left(1-r^{2}\right)}{\pi} \int_{0}^{\pi / 2} \frac{\sin ^{2 \lambda-1} t}{D^{\lambda+1}} d t
$$

Then

$$
P(r, \theta, \phi)=P^{+}(r, \theta, \phi)+P^{+}(r, \theta,-\phi) .
$$

We shall now prove the estimate (12.5) for $P_{r}^{+}(r, \theta, \phi)$. In fact

(12.8) $P_{r}^{+}(r, \theta, \phi)=\frac{-2 \lambda r}{\pi} \int_{0}^{\pi / 2} \frac{\sin ^{2 \lambda-1} t}{D^{\lambda+1}} d t-\frac{\lambda(\lambda+1)}{\pi}\left(1-r^{2}\right) \int_{0}^{\pi / 2} \sin ^{2 \lambda-1} t \frac{D_{r}}{D^{\lambda+2}} d t$.

(6) When $0 \leqq r \leqq 1 / 2$ the trivial estimate $\left|P_{r}(r, \theta, \phi)\right| \leqq A$ will suffice. 
To study the situation further we shall concentrate on the main singularity, which occurs when $\theta$ is near $\phi$ (and $t$ is near zero) in the integrals in (12.8).

Thus we write $\Delta^{*}=\Delta+t^{2} r \sin \theta \sin \phi=\Delta+t^{2} \sigma$. Since $D=\Delta+2 \sigma(1-\cos t)$, it follows that $D=\Delta^{*}+O\left(\sigma t^{4}\right)$. Notice that $D_{r}-\Delta_{r}=O\left(\sigma t^{4}\right)$.

In this way, we are led to replace systematically $D$ by $\Delta^{*}, \sin ^{2 \lambda-1} t$ by $t^{2 \lambda-1}$, and $D_{r}$ by $\Delta_{r}$. We therefore define $P_{r}^{*}(r, \theta, \phi)$ by

$$
P_{r}^{*}(r, \theta, \phi)=\frac{-2 \lambda r}{\pi} \int_{0}^{\pi / 2} \frac{t^{2 \lambda-1}}{\left(\Delta^{*}\right)^{\lambda+1}} d t-\frac{\lambda(\lambda+1)\left(1-r^{2}\right) \Delta_{r}}{\pi} \int_{0}^{\pi / 2} \frac{t^{2 \lambda-1}}{\left(\Delta^{*}\right)^{\lambda+2}} d t
$$

Let us try to determine by how much $P_{r}^{*}(r, \theta, \phi)$ actually differs from $P_{r}^{+}(r, \theta, \phi)$ (see (12.8)). Now by the mean value theorem

$$
[\Delta+2 \sigma(1-\cos t)]^{-\lambda-1}-\left[\Delta+\sigma t^{2}\right]^{-\lambda-1}=O\left[\Delta+\sigma t^{2}\right]^{-\lambda-2} \sigma t^{4},
$$

since $\Delta \geqq 0, \sigma \geqq 0$ and $t \geqq 0$. In other words

$$
D^{-\lambda-1}-\left(\Delta^{*}\right)^{-\lambda-1}=O\left(\Delta^{*}\right)^{-\lambda-2} \sigma t^{4} .
$$

Similarly

$$
D^{-\lambda-2}-\left(\Delta^{*}\right)^{-\lambda-2}=O\left(\Delta^{*}\right)^{-\lambda-3} \sigma t^{4} .
$$

Also we should point out that since $\Delta=(1-r)^{2}+2 r(1-\cos (\theta-\phi))$ and $1 / 2 \leqq r<1$, then $\left|(1-r) \Delta_{r}\right| \leqq 4 \Delta$.

Let us now define

$$
I_{\mu}^{\nu}=\int_{0}^{\pi / 2} \frac{t^{\nu}}{\left(\Delta^{*}\right)^{\mu}} d t
$$

Considering what has been said above, and after some simple reductions, we obtain that the difference $P_{r}^{+}(r, \theta, \phi)-P_{r}^{*}(r, \theta, \phi)$ is bounded by a constant multiple of

$$
\begin{aligned}
(1-r) \Delta I_{\lambda+2}^{2 \lambda-1}+(1-r) \sigma I_{\lambda+2}^{2 \lambda+1} & +(1-r) \sigma^{2} I_{\lambda+3}^{2 \lambda+3} \\
& +\Delta I_{\lambda+2}^{2 \lambda+1}+\Delta \sigma I_{\lambda+3}^{2 \lambda+3}+I_{\lambda+1}^{2 \lambda+1}+\sigma I_{\lambda+2}^{2 \lambda+3} .
\end{aligned}
$$

By a change of variables

$$
I_{\mu}^{\nu}=\sigma^{-\nu-1 / 2} \Delta^{\nu / 2-\mu+1 / 2} \int_{0}^{\xi} \frac{t^{\nu}}{\left(1+t^{2}\right)^{\mu}} d t, \text { where } \xi=\frac{\pi}{2}\left(\frac{\sigma}{\Delta}\right)^{1 / 2} .
$$

However,

$$
\int_{0}^{\xi} \frac{t^{\nu}}{\left(1+t^{2}\right)^{\mu}} d t \leqq A\left(\frac{\xi}{1+\xi}\right)^{\nu+1} \text { if } v>-1, v-2 \mu<-1 ;
$$

also 


$$
\int_{0}^{\xi} \frac{t^{v}}{\left(1+t^{2}\right)^{\mu}} d t \leqq A\left(\frac{\xi}{1+\xi}\right)^{v+1}\left[1+\log ^{+} \xi\right] \text { if } v>-1, v-2 \mu=-1 .
$$

From this we get by straightforward calculation the following estimates:

$$
\begin{aligned}
& I_{\lambda+2}^{2 \lambda-1}=O\left(\sigma^{-\lambda} \Delta^{-2}\right)(\lambda>0)(\text { a more precise form is given below }) \\
& I_{\lambda+2}^{2 \lambda+1}=O\left(\sigma^{-\lambda} \Delta^{-1}(\sigma+\Delta)^{-1}\right)=O\left(\sigma^{-\lambda-1} \Delta^{-1}\right) \\
& I_{\lambda+3}^{2 \lambda+3}=O\left(\sigma^{-\lambda-1} \Delta^{-1}(\sigma+\Delta)^{-1}\right)=O\left(\sigma^{-\lambda-2} \Delta^{-1}\right) \\
& I_{\lambda+2}^{2 \lambda+3}=O\left(\sigma^{-\lambda-1} \Delta^{-1}\right) \\
& I_{\lambda+1}^{2 \lambda+1}=O\left(\sigma^{-\lambda}(\sigma+\Delta)^{-1}\left(1+\log ^{+}(\sigma / \Delta)\right)\right)
\end{aligned}
$$

Therefore we see the total contribution in (12.11) is bounded by a constant multiple of

$$
\sigma^{-\lambda}(1-r) \Delta^{-1}+\frac{\sigma^{-\lambda}}{\sigma+\Delta}\left(1+\log ^{+}(\sigma / \Delta)\right)
$$

To analyze $P_{r}^{*}(r, \theta, \phi)$ we need to look at the integrals $I_{\lambda+1}^{2 \lambda-1}$ and $I_{\lambda+2}^{2 \lambda-1}$ more closely (see (12.9)).

Recall that

$$
I_{\mu}^{\nu}=\sigma^{-\nu-1 / 2} \Delta^{\nu / 2-\mu+1 / 2} \int_{0}^{\xi} \frac{t^{\nu}}{\left(1+t^{2}\right)^{\mu}} d t, \text { where } \xi=\frac{\pi}{2}\left(\frac{\sigma}{\Delta}\right)^{1 / 2} .
$$

But

$$
\int_{0}^{\xi} \frac{t^{\nu}}{\left(1+t^{2}\right)^{\mu}} d t=\int_{0}^{\infty} \frac{t^{\nu}}{\left(1+t^{2}\right)^{\mu}} d t-\int_{\xi}^{\infty} \frac{t^{\nu}}{\left(1+t^{2}\right)^{\mu}} \text { if } v-2 \mu<-1 \text {. }
$$

From this, and a well-known formula in the theory of the gamma function, it follows that

and

$$
I_{\lambda+1}^{2 \lambda-1}=(2 \lambda)^{-1} \sigma^{-\lambda} \Delta^{-1}+O\left(\frac{\sigma^{-\lambda}}{(\sigma+\Delta)}\right)
$$

$$
I_{\lambda+2}^{2 \lambda-1}=(\lambda(\lambda+1))^{-1} \sigma^{-\lambda} \Delta^{-2}+O\left(\sigma^{-\lambda} \frac{\Delta^{-1}}{(\sigma+\Delta)}\right) .
$$

Combining all the above via the estimate in (12.12) gives us (12.5) and the lemma is proved.

We come now to the proof of Theorem 8 .

Proof of (12.2). Recall that

where

$$
g(\theta, f)=\left(\int_{0}^{1}(1-r)\left|f_{r}(r, \theta)\right|^{2} d r\right)^{1 / 2},
$$




$$
f_{r}(r, \theta)=\int_{0}^{\pi} P_{r}(r, \theta, \phi) f(\phi) \sin ^{2 \lambda} \phi d \phi .
$$

Thus we have $g(\theta, f) \leqq g_{1}(\theta, f)+g_{2}(\theta, f)+g_{3}(\theta, f)+g_{4}(\theta, f)$. $g_{k}(\theta, f)$ is defined like $g(\theta, f)$, where $P_{r}(r, \theta, \phi)$ is replaced by $P_{r}^{(k)}(r, \theta, \phi)$, and

$$
\begin{aligned}
P_{r}^{(1)}(r, \theta, \phi) & =P_{r}(r, \theta, \phi) & & \text { if } 0 \leqq r \leqq 1 / 2, \\
& =0 & & \text { otherwise; } \\
P_{r}^{(2)}(r, \theta, \phi) & =P_{r}(r, \theta, \phi) & & \text { if } 1 / 2 \leqq r<0 \text { and } 0 \leqq \phi \leqq(3 / 4) \theta, \\
& =0 & & \text { otherwise; } \\
P_{r}^{(3)}(r, \theta, \phi) & =P_{r}(r, \theta, \phi) & & \text { if } 1 / 2 \leqq r<0 \text { and }(4 / 3) \theta \leqq \phi \leqq \pi, \\
& =0 & & \text { otherwise; } \\
P_{r}^{(4)}(r, \theta, \phi) & =P_{r}(r, \theta, \phi) & & \text { if } 1 / 2 \leqq r<0 \text { and }(3 / 4) \theta<\phi<(4 / 3) \theta .
\end{aligned}
$$

We need to prove, therefore, that $\left\|g_{k}(\theta, f)\right\|_{p} \leqq A_{p}\|f\|_{p}, k=1,2,3,4$.

The inequality $\left\|g_{1}(\theta, f)\right\|_{p} \leqq A\|f\|_{p}$ is trivial since $P_{r}^{1}(r, \theta, \phi)$ is uniformly bounded.

To prove the other three inequalities, we notice that it suffices to assume that $f(\phi)=0$ when $\phi \in[\pi / 2, \pi]$, because $P_{r}(r, \theta, \phi)=P_{r}(r, \pi-\theta, \pi-\phi)$ makes the consideration of the intervals $[0, \pi / 2]$ and $[\pi / 2, \pi]$ completely symmetric.

Now by estimate (12.4) of $P_{r}(r, \theta, \phi)$, we see that

$$
\left|P_{r}^{(2)}(r, \theta, \phi)\right| \leqq C\left[(1-r)^{2}+2 r(1-(\cos \theta-\phi))\right]^{-\lambda-1} \leqq C\left(\left(1-r^{2}\right)+\theta\right)^{2 /-\lambda-1},
$$

since $0 \leqq \phi \leqq(3 / 4) \theta$. Hence

$$
\begin{aligned}
g_{2}(\theta, f) & =\int_{1 / 2}^{1}(1-r)\left|\int_{0}^{(3 / 4) \theta} P_{r}^{(2)}(r, \theta, \phi) f(\phi) \sin ^{2 \lambda} \phi d \phi\right|^{2} d r \\
\leqq & C \int_{1 / 2}^{1}(1-r)\left[(1-r)^{2}+\theta^{2}\right]^{-2 \lambda-2}\left[\int_{0}^{(3 / 4) \theta}|f(\phi)|^{p} \sin ^{2 \lambda} \phi d \phi\right]^{p / 2} \theta^{2 / q+1} d r
\end{aligned}
$$

by Hölder's inequality. If we carry out the integration with respect to $r$ we obtain

Thus

$$
g^{2}(\theta, f) \leqq C \theta^{2 / q-2(2 \lambda+1)}\left[\int_{0}^{(3 / 4) \theta}|f(\phi)|^{p} \sin ^{2 \lambda p} \phi d \phi\right]^{p / 2} .
$$

$$
\begin{aligned}
\int_{0}^{\pi}\left(g_{2}(\theta, f)\right)^{p} \sin ^{2 \lambda} \theta d \theta & \leqq C \int_{0}^{\pi} i \theta^{-1-2 \lambda p+2 \lambda} d \theta \int_{0}^{(3 / 4) \theta}|f(\phi)|^{p} \sin ^{2 \lambda p} \phi d \phi \\
& \leqq C \int_{0}^{\pi / 2}|f(\phi)|^{p} \sin ^{2 \lambda p} \phi d \phi \int_{(4 / 3) \theta}^{\pi} \phi^{-1-2 \lambda p+2 \lambda} d \phi \\
& \leqq C \int_{0}^{\pi}|f(\phi)|^{p} \sin ^{2 \lambda} \phi d \phi,
\end{aligned}
$$

since $f(\phi)=0$ on $[\pi / 2, \pi]$. 
By very similar arguments one can also prove that

$$
\left\|g_{3}(\theta, f)\right\|_{p} \leqq A\|f\|_{p} \text {. }
$$

We now come to the case of $g_{4}(\theta, f)$. For its study we shall use the asymptotic estimate (12.5) for $P_{r}(r, \theta, \phi)$. We notice that this leads to two terms, one for $P_{r}^{+}$ and the other for $P_{r}^{+}(r, \theta,-\phi)$. Again considerations of symmetry reduce the problem to that of $P_{r}^{+}(r, \theta, \phi)$.

So we are led to consider the three functions, $l(\theta), m(\theta)$, and $n(\theta)$ defined respectively by

$$
\begin{aligned}
& {[l(\theta)]^{2}=\left[\int_{1 / 2}^{1}(1-r) \int_{(3 / 4) \theta}^{(4 / 3) \theta} \sigma^{-\lambda} \frac{\partial}{\partial r}\left(\frac{1-r^{2}}{\Delta}\right) f(\phi) \sin ^{2 \lambda} \phi d \phi\right]^{2} d r,} \\
& {[m(\theta)]^{2}=\int_{1 / 2}^{1}(1-r)\left[\int_{(3 / 4) \theta}^{(4 / 3) \theta}(1-r) \sigma^{-\lambda \Delta^{-1}}|f(\phi)| \sin ^{2 \lambda} \phi d \phi\right]^{2} d r}
\end{aligned}
$$

and

$$
[n(\theta)]^{2}=\int_{1 / 2}^{2}(1-r)\left[\int_{(3 / 4) \theta}^{(4 / 3) \theta}(1-r)\left(\frac{\sigma^{-\lambda}}{(\sigma+\Delta)}\right)\left(1+\log ^{+}\left(\frac{\sigma}{\Delta}\right)\right)|f(\phi)| \sin ^{2 \lambda} \phi d \phi\right]^{2} d r .
$$

We need to prove that

$$
\|n(\theta)\|_{p} \leqq A_{p}\|f\|_{p}
$$

with similar inequalities for $m$ and $l$. Consider $n(\theta)$ first. When $(3 / 4) \theta \leqq \phi \leqq(4 / 3) \theta, 0 \leqq \phi \leqq \pi / 2$, we have the simple estimates $\sigma^{-\lambda} \leqq C \theta^{-2 \lambda}$, $\sigma+\Delta \geqq C\left[\theta^{2}+(1-r)^{2}\right], \Delta \geqq C(\theta-\phi)^{2}$, and $\sigma \geqq C \theta \phi$. Substitute these estimates, integrate with respect to $r$ and use the fact that

$$
\int_{1 / 2}^{1} \frac{(1-r)}{\left(\theta^{2}+(1-r)^{2}\right)^{2}} d r=O\left(\theta^{-2}\right) \text {. }
$$

Then we get

$$
n(\theta) \leqq C \theta^{-1} \int_{(3 / 4) \theta}^{(4 / 3) \theta}\left[1+\log ^{+}\left(\frac{\phi}{\theta-\phi}\right)^{2}\right]|f(\phi)| d \phi .
$$

But then by Hölder's inequality

$\int_{0}^{\pi}(n(\theta))^{p} \sin ^{2 \lambda} \theta d \theta \leqq \int_{0}^{\pi} \theta^{2 \lambda-p} \int_{(3 / 4) \theta}^{(4 / 3) \theta}|f(\phi)|^{p} d \phi\left\{\int_{(3 / 4) \theta}^{(4 / 3) \theta}\left[1+\log ^{+}\left(\frac{\phi}{\theta-\phi}\right)^{2}\right]^{q} d \phi\right\}^{p / q} d \theta$, where $1 / p+1 / q=1$. However,

$$
\int_{(3 / 4) \theta}^{(4 / 3) \theta}\left[1+\log ^{+}\left(\frac{\phi}{\theta-\phi}\right)^{2}\right]^{q} d \phi=\theta \cdot \int_{3 / 4}^{4 / 3}\left[1+\log ^{+}\left(\frac{\phi}{1-\phi}\right)^{2}\right]^{q} d \phi .
$$


Thus

$$
\int_{0}^{\pi}(n(\theta))^{p} \sin ^{2 \lambda} \theta d \theta \leqq C \int_{0}^{\pi} \theta^{2 \lambda-1} \int_{(3 / 4) \theta}^{(4 / 3) \theta}|f(\phi)|^{p} d \phi \leqq C \int_{0}^{\pi}|f(\phi)|^{p} \sin ^{2 \lambda} \phi d \phi,
$$

since

$$
f(\phi)=0 \quad \text { when } \phi \in[\pi / 2, \pi] .
$$

We now consider $m(\theta)$, and therefore we define $F_{r}(\theta)$ by

$$
F_{r}(\theta)=(1-r) \int_{(3 / 4) \theta}^{(4 / 3) \theta} \sigma^{-\lambda} \Delta^{-1}|f(\phi)| \sin ^{2 \lambda} \phi d \phi .
$$

Since $\sigma=r \sin \theta \sin \phi$, and $f(\phi)=0$ if $\phi>\pi / 2$, we get

$$
\begin{aligned}
F_{r}(\theta)(\sin \theta)^{2 \lambda / p} & \leqq C\left(1-r^{2}\right) \int_{(3 / 4) \theta}^{(4 / 3) \theta} \Delta^{-1}|f(\phi)|(\sin \phi)^{2 \lambda / p} d \phi \\
& \leqq C\left(1-r^{2}\right) \int_{0}^{\pi} \Delta^{-1}|f(\phi)|(\sin \phi)^{2 \lambda / p} d \phi .
\end{aligned}
$$

Thus, by a well-known property of the ordinary Poisson kernel, $\left(1-r^{2}\right) / \Delta$ we have

$$
\int_{0}^{\pi}\left[F_{r}(\theta)\right]^{p} \sin ^{2 \lambda}(\theta) d \theta \leqq C \int_{0}^{\pi}\left|f(\phi)^{p}\right| \sin ^{2 \lambda} \phi d \phi,
$$

with $C$ independent of $r, 0 \leqq r<1$.

But

$$
m(\theta)=\left(\int_{1 / 2}^{1}(1-r)\left[F_{r}(\theta)\right]^{2} d r\right)^{1 / 2} .
$$

Therefore Minkowski's inequality for integrals and the above shows that

$$
\int_{0}^{\pi}(m(\theta))^{p} \sin ^{2 \lambda} \theta d \theta \leqq C \int_{0}^{\pi}|f(\phi)|^{p} \sin ^{2 \lambda} \phi d \phi .
$$

Finally we come to $l(\theta)$, and here the argument is somewhat intricate.

Since in our case we always have $(3 / 4) \theta \leqq \phi \leqq(4 / 3) \theta$, and $0 \leqq \phi \leqq \pi / 2$ (i.e. $f(\phi)=0$ if $\pi / 2<\phi \leqq \pi)$, then we may limit ourselves to $\theta \in[0,(4 / 3) \cdot \pi / 2]$. Thus we define the intervals $E_{n}=\left[(4 / 3) \cdot \pi / 2^{n-1},(4 / 3) \cdot \pi / 2^{n}\right]$; hence $\bigcup_{n=1}^{\infty} E_{n}=[0,(4 / 3) \cdot \pi / 2]$. The $E_{n}$ 's are the appropriate intervals for the $\theta$ variable. The corresponding intervals for the $\phi$ variable are gotten by multiplying the right-hand end point by $4 / 3$ and the left-hand end point by $3 / 4$. In this way we define $E_{n}^{*}=\left[\pi / 2^{n-1},(4 / 3)^{2} \cdot \pi / 2^{n}\right]$. Notice that while the sets $E_{n}$ are essentially nonoverlapping, the sets $E_{n}^{*}$ overlap at most twice. 
Now we have

$$
(l(\theta))^{2}=\int_{1 / 2}^{1}(1-r)\left|G_{r}(\theta)\right|^{2} d r
$$

where

$$
G_{r}(\theta)=\int_{(3 / 4) \theta}^{(4 / 3) \theta} \sigma^{-\lambda} \frac{\partial}{\partial r}\left(\frac{\left(1-r^{2}\right)}{\Delta}\right) f(\phi) \sin ^{2 \lambda} \phi d \phi=\int_{(3 / 4) \theta}^{(4 / 3) \theta} I(\phi) d \phi .
$$

In view of the above we may write this as

$$
G_{r}(\theta)=\sum_{n=1}^{\infty} \chi_{E_{n}}(\theta) \int_{(3 / 4) \theta}^{(4 / 3) \theta} \chi_{E_{n}^{*}}(\phi) I(\phi) d \phi,
$$

where $\chi_{E}$ denotes the characteristic function of the set $E$. We should like, however, to replace the above sum by

$$
G_{r}^{*}(\theta)=\sum_{n=1}^{\infty} \chi_{E_{n}}(\theta) \int_{E_{n}^{*}} I(\phi) d \phi
$$

But the difference between $G_{r}(\theta)$ and $G_{r}^{*}(\theta)$ is bounded by

where

$$
\sum_{n=1}^{\infty} \chi_{E_{n}}(\theta) \int_{E_{n}^{\prime}}|I(\phi)| d \phi
$$

$$
E_{n}^{\prime}=\left[\pi / 2^{n-1},(3 / 4) \theta\right] \cup\left[(4 / 3) \theta,(4 / 3)^{2} \cdot \pi / 2^{n}\right]
$$

From the definitions of the sets $E_{n}, E_{n}^{*}$ and their overlapping character, it follows that

$$
\left|G_{r}(\theta)-G_{r}^{*}(\theta)\right| \leqq 2 \int_{(3 / 8) \theta}^{(3 / 4) \theta}|I(\phi)| d \phi+2 \int_{(4 / 3) \theta}^{(8 / 3) \theta}|I(\phi)| d \phi .
$$

For the range of $\phi$ 's appearing in the above it is easy to see that

$$
\frac{\partial}{\partial r}\left(\frac{\left(1-r^{2}\right)}{\Delta}\right)=O\left(\left(\theta^{2}+(1-r)^{2}\right)^{-1}\right) .
$$

In effect we are led back to the argument used to estimate $n(\theta)$ above. By that argument, we get

$$
\left(\int_{1 / 2}^{1}(1-r)\left|G_{r}(\theta)-G_{r}^{*}(\theta)\right|^{2} d r\right)^{1 / 2} \leqq C \theta^{-1}\left[\int_{(3 / 8) \theta}^{(3 / 4) \theta}|f(\phi)| d \phi+\int_{(4 / 3) \theta}^{(8 / 3) \theta}|f(\phi)| d \phi\right] .
$$

(See the argument leading to (12.13).) 
Following through as before, we obtain

$$
\left\|\left(\int_{1 / 2}^{1}(1-r)\left|G_{r}(\theta)-G_{r}^{*}(\theta)\right|^{2} d r\right)^{1 / 2}\right\|_{p} \leqq A\|f\|_{p} .
$$

Hence we have reduced everything to proving that

$$
\left\|\left(\int_{1 / 2}^{1}(1-r)\left|G_{r}^{*}(\theta)\right|^{2} d r\right)^{1 / 2}\right\|_{p} \leqq A_{p}\|f\|_{p}
$$

However, by definition (12.14),

$$
\begin{aligned}
& \left\|\left(\int_{1 / 2}^{1}(1-r)\left|G_{r}^{*}(\theta)\right|^{2} d r\right)^{1 / 2}\right\|_{p}^{p} \\
& =\sum_{n=1}^{\infty} \int_{E_{n}}\left[\int_{1 / 2}^{1}(1-r)\left|\int_{E_{n}^{*}} \sigma^{-\lambda} \frac{\partial}{\partial r}\left(\frac{\left(1-r^{2}\right)}{\Delta}\right) f(\phi) \sin ^{2 \lambda} \phi d \phi\right|^{2} d r\right]^{p / 2} \sin ^{2 \lambda} \theta d \theta \\
& \leqq C \sum_{n=1}^{\infty} 2^{-n(2 \lambda-p \lambda)} \int_{E_{n}}\left[\int_{0}^{1}(1-r)\left|\int_{0}^{\pi} f(\phi) \sin ^{2 \lambda} \phi \chi_{E_{n}^{*}}(\phi) \frac{\partial}{\partial r}\left(\frac{\left(1-r^{2}\right)}{\Delta}\right) d \phi\right|^{2} d r\right]^{p / 2} d \theta
\end{aligned}
$$

since $\sigma=r \sin \theta \sin \phi$.

Let us now use the classical inequality for the $g$-function $\left({ }^{7}\right)$, which states in particular

$$
\begin{gathered}
\int_{0}^{\pi}\left[\int_{0}^{1}(1-r)\left|\int_{0}^{\pi} f(\phi) \sin ^{\lambda} \phi \chi E_{n}^{*}(\phi) \frac{\partial}{\partial r}\left(\frac{\left(1-r^{2}\right)}{\Delta}\right) d \phi\right|^{2} d r\right]^{p / 2} d \theta \\
\leqq C \int_{0}^{\pi}\left|f(\phi)(\sin \phi)^{\lambda}\right|{ }^{p} \chi_{E_{n}^{*}}(\phi) d \phi=C \int_{E_{n}^{*}}|f(\phi)|^{p} \sin ^{\lambda p} \phi d \phi .
\end{gathered}
$$

Combining this with the above gives us

$$
\begin{aligned}
\left\|\left(\int_{1 / 2}^{1}(1-r)\left|G_{r}^{*}(\theta)\right|^{2} d r\right)^{1 / 2}\right\|_{p}^{p} & \leqq C \sum_{n=1}^{\infty} 2^{-n(2 \lambda-p \lambda)} \int_{E_{n}^{*}}|f(\phi)|^{p} \sin ^{\lambda p} \phi d \phi \\
& \leqq C \int_{0}^{\pi}|f(\phi)|^{p} \sin ^{2 \lambda} \phi d \phi .
\end{aligned}
$$

This proves (12.5), and therefore the proof of the direct part of the theorem (12.2) is complete.

Proof of (12.3). The whole matter here is rather simple and is based on the following "duality": if $\int_{0}^{\pi} f_{1}(\theta) \sin ^{2 \lambda} \theta d \theta=0$,

$$
\left|\int_{0}^{\pi} f_{1}(\theta) f_{2}(\theta) \sin ^{2 \lambda} \theta d \theta\right| \leqq 4 \int_{0}^{\pi} g\left(\theta, f_{1}\right) g\left(\theta, f_{2}\right) \sin ^{2 \lambda} \theta d \theta .
$$

(7) See [26, Chapter 14]. 
This holds whenever $f_{1}(\theta)$ and $f_{2}(\theta)$ are regular enough, and in particular when $f_{1}$ and $f_{2}$ have finite expansions.

In fact, let $f_{1}(\theta) \sim \sum_{n=1} a_{n} P_{n}(\cos \theta)$, and $f_{2}(\theta) \sim \sum_{n=0} b_{n} P_{n}(\cos \theta)$.

Set $\left\langle f_{1}, f_{2}\right\rangle=\int_{0}^{\pi} f_{1}(\theta) f_{2}(\theta) \sin ^{2 \lambda} \theta d \theta$.

We then have $\left\langle f_{1}, f_{2}\right\rangle=\sum_{n=1}^{\infty} a_{n} b_{n} \gamma_{n}^{-1}$, where $\gamma_{n}$ are the normalizing constants. However,

$$
\sum_{n=1} a_{n} b_{n} \gamma_{n}^{-1}=4 \int_{0}^{1} \frac{1}{\rho}\left\{\int_{0}^{r}\left(\sum_{n=1} a_{n} b_{n} \gamma_{n}^{-1} n^{2} r^{2 n-1}\right) d r\right\} d \rho .
$$

This means that

$$
\left\langle f_{1}, f_{2}\right\rangle=4 \int_{0}^{1} \frac{1}{\rho}\left\{\int_{0}^{r}\left\langle\frac{\partial f_{1}}{\partial r}, \frac{\partial f_{2}}{\partial r}\right\rangle r d r\right\} d \rho .
$$

We have set $f_{1}(r, \theta)=\sum a_{n} r^{n} P_{n}^{\lambda}(\cos \theta)$, and $\partial f_{1} / \partial r=\sum n a_{n} r^{n-1} P_{n}^{\lambda}(\cos \theta)$, with a similar notation for $f_{2}$.

We therefore obtain that

$$
\left|\left\langle f_{1}, f_{2}\right\rangle\right| \leqq 4 \int_{0}^{1}(1-r)\left\langle\frac{\partial f_{1}}{\partial r}, \frac{\partial f_{2}}{\partial r}\right\rangle d r .
$$

Applying Fubini's theorem and Schwarz's inequality gives (12.16).

Next, (12.16) extends to the case when $f_{1} \in L^{p}, 1<p<\infty, \int_{0}^{\pi} f_{1}(\theta) \sin ^{2 \lambda} \theta d \theta=0$, and $f_{2} \in L^{q}, 1 / p+1 / q=1$.

In fact, we already know that the sub-linear mapping $f \rightarrow g(f)$ is bounded in $L^{p}$ (and $L^{q}$ ); moreover its sub-linearity implies that

$$
\left|g\left(f_{1}^{(n)}\right)-g\left(f_{1}^{(m)}\right)\right| \leqq g\left(f_{1}^{n}-f_{1}^{m}\right) .
$$

Thus if $\left\{f_{1}^{n}\right\}$ is a convergent sequence in the $L^{p}$ norm, so is $\left\{g\left(f_{1}^{n}\right)\right\}$. Hence a passage to the limit shows that (12.16) holds whenever $f_{1} \in L^{p}$ and $f_{2} \in L^{q}, 1 / p+1 / q-1, \int_{0}^{\pi} f_{1}(\theta) \sin ^{2 \lambda} \theta d \theta=0$.

Finally

$$
\left\|f_{1}\right\|_{p}=\sup _{\left\|f_{2}\right\|_{g} \leqq 1}\left|\int_{0}^{\pi} f_{1}(\theta) f_{2}(\theta) \sin ^{2 \lambda} \theta d \theta\right| \leqq 4\left\|g\left(\theta, f_{1}\right)\right\|_{p}\left\|g\left(\theta, f_{2}\right)\right\|_{q} \leqq 4 A_{q}\left\|g\left(\theta, f_{1}\right)\right\|_{p},
$$

by (12.2). This proves (12.3) and the proof of our theorem is complete.

13. Partial sums analogue of the $g$-function. The $g$-function which was studied in the previous section is intimately connected with the Poisson integral

$$
\begin{aligned}
f(r, \theta) & =\sum_{k=0}^{\infty} a_{k} r^{k} P_{k}^{\lambda}(\cos \theta), \\
f(\theta) & \sim \sum_{k=0}^{\infty} a_{k} P_{k}^{\lambda}(\cos \theta) .
\end{aligned}
$$


It is, therefore, closely related to the Abel-summability of the expansion in question. What we need, however, are the analogous facts for ordinary convergence instead of Abel-summability. This is the result obtained in the theorem of this section; thus what follows below may be considered as an argument of Tauberian character. Let us look at the situation more closely. Instead of the Abel means (13.1), we consider the ordinary partial sums

$$
S_{n}(\theta)=S_{n}(\theta, f)=\sum_{k=0}^{n} a_{k} P_{k}^{\lambda}(\cos \theta)
$$

Similarly in place of $f_{r}(r, \theta)=\sum k a_{k} r^{k-1} P_{k}^{\lambda}(\cos \theta)$, we consider

$$
S_{n}^{\prime}(\theta)=S_{n}^{\prime}(\theta, f)=\sum_{k=1}^{n} k a_{k} P_{k}^{\lambda}(\cos \theta) .
$$

Moreover, as is familiar, $n$ and $r$ are to be connected by $1 / n=1-r$.

Therefore, instead of

$$
g(\theta)=\left(\int_{0}^{1}(1-r)\left|f_{r}(r, \theta)\right|^{2} d r\right)^{1 / 2}
$$

we are led to consider its analogue

$$
g^{*}(\theta)=g^{*}(\theta, f)=\left(\sum_{n=1}^{\infty} \frac{\left|S_{n}^{\prime}(\theta, f)\right|^{2}}{n^{3}}\right)^{1 / 2} .
$$

If we recall the argument of the classical Abelian theorem, we are led to expect an inequality of the form

$$
g(\theta) \leqq c g^{*}(\theta) .
$$

Indeed, we shall see that this is the case. In the converse direction, we cannot hope for anything as simple as (13.5). However, it will turn out that (13.5) may be reversed not for individual $\theta$ 's, but as a whole, in the sense of an $L^{p}$ inequality. We must add one further reservation to this, which arises because the partial sums inequalities of $\$ 10$ are limited to the case when the exponent $p$ satisfies $(2 \lambda+1) /(\lambda+1)<p<(2 \lambda+1) / \lambda$. For this reason we may expect the result

$$
\left\|g^{*}(\theta)\right\|_{p} \leqq A_{p}\|g(\theta)\|_{p}, \quad \frac{2 \lambda+1}{\lambda+1}<p<\frac{2 \lambda+1}{\lambda} .
$$

We formulate the above as a theorem.

THEOREM 9. The inequalities (13.5) and (13.6) hold.

We prove (13.5) first. 
Since $f_{r}(r, \theta)=\sum k a_{k} r^{k-1} P_{k}^{\lambda}(\cos \theta)$ and $S_{n}^{\prime}(\theta)=\sum_{k=1}^{n} k a_{k} P_{k}^{\lambda}(\cos \theta)$, it follows that

$$
f_{r}(r, \theta)=(1-r) \sum_{k=1}^{\infty} S_{k}^{\prime}(\theta) r^{k-1}
$$

By Schwarz's inequality

$$
\begin{aligned}
\left|f_{r}(r, \theta)\right|^{2} & \leqq(1-r)^{2}\left(\sum_{k=1}^{\infty}\left|S_{k}^{\prime}(\theta)\right|^{2} r^{k-1}\right) \sum_{k=1}^{\infty} r^{k-1} \\
& =(1-r) \sum_{k=1}^{\infty}\left|S_{k}^{\prime}(\theta)\right|^{2} r^{k-1} .
\end{aligned}
$$

Hence

because

$$
\int_{0}^{1}(1-r)\left|f_{r}(r, \theta)\right|^{2} d r \leqq c \sum_{k=1}^{\infty} \frac{\left|S_{k}^{\prime}(\theta)\right|^{2}}{k^{3}}
$$

$$
\int_{0}^{1}(1-r)^{2} r^{k-1} d r \approx 1 / k^{3}
$$

This proves (13.5).

The inequality (13.6) lies deeper, because unlike (13.5) it makes use of Theorem 7 on partial sums, more particularly its corollary. In proving (13.6) we follow the argument in [26, Chapter 15] closely.

We have

$$
S_{n}^{\prime}(\theta)=\sum_{k=1}^{n} k a_{k} P_{k}^{\lambda}(\cos \theta)=\sum_{k=1}^{n} k a_{k} r^{k-1} P_{k}^{\lambda}(\cos \theta) r^{1-k} .
$$

Sum the last expression by parts, set $r=r_{n}=1-1 / n$, and use Schwarz's inequality. This gives

$$
\left|S_{n}^{\prime}(\theta)\right|^{2} \leqq A\left\{n^{-1} \sum_{k=1}^{n-1}\left|S_{k}^{\prime}\left(\theta, f_{n}\right)\right|^{2}+\left|S_{n}^{\prime}\left(\theta, f_{n}\right)\right|^{2}\right\},
$$

where $f_{n}=f\left(r_{n}, \theta\right), r_{n}=1-1 / n$.

But

$$
\begin{aligned}
g^{*}(\theta) & =\sum_{k=1}^{\infty} \frac{\left|S_{n}^{\prime}(\theta)\right|^{2}}{n^{3}} \\
& \leqq A\left\{\sum_{n=1}^{\infty} n^{-4} \sum_{k=1}^{n-1}\left|S_{k}^{\prime}\left(\theta, f_{n}\right)\right|^{2}+\sum_{n=1}^{\infty} \frac{\left|S_{n}^{\prime}\left(\theta, f_{n}\right)\right|^{2}}{n^{3}}\right\}=G_{1}(\theta)+G_{2}(\theta)
\end{aligned}
$$

If we apply the corollary to Theorem 7 (see §11) we obtain

$$
\left\|G_{1}(\theta)\right\|_{p} \leqq A_{p}\left\|\left\{\sum_{n=1}^{\infty} n^{-4} \sum_{k=1}^{n-1}\left|\delta_{n}^{-1}\right| \int_{\delta_{n}}\left|f_{r}(r, \theta)\right|^{2} d r\right\}^{1 / 2}\right\|_{p},
$$

where $\delta_{n}=\left(r_{n}, r_{n+1}\right)$, and $(2 \lambda+1) /(\lambda+1)<p<(2 \lambda+1) / \lambda$. 
But $\left|\delta_{n}\right|=r_{n+1}-r_{n}=1 /(n+1)-1 / n=1 / n(n+1) \geqq 1 /(n+1)^{2}$. Hence,

$$
\begin{aligned}
\left\|G_{1}(\theta)\right\|_{p} & \leqq A_{p}\left\|\left\{\sum_{n=1}^{\infty} n^{-1} \int_{\delta_{n}}\left|f_{r}(r, \theta)\right|^{2} d r\right\}^{1 / 2}\right\|_{p} \\
& \leqq A_{p}\left\|\left(\int_{0}^{1}(1-r)\left|f_{r}(r, \theta)\right|^{2} d r\right)^{1 / 2}\right\|_{p}=A_{p}\|g\|_{p} .
\end{aligned}
$$

Similarly for $G_{2}(\theta)$.

This proves (13.6) and hence the theorem.

The following corollary is an immediate consequence of the above theorem and Theorem 8 in the previous section.

Corollary 1. If $(2 \lambda+1) /(\lambda+1)<p<(2 \lambda+1) / \lambda$, then $\|f(\theta)\|_{p}$ and $\left\|_{\pi} g^{*}(\theta, f)\right\|_{p}$ are equivalent norms for the class of functions normalized by $\int_{0} f(\theta) \sin ^{2 \lambda} \theta d \theta=0$.

Another corollary that can be deduced is in effect a generalization of the theorem.

Let $v_{1}, v_{2}, \cdots, v_{n}, \cdots$ be a series of positive constants satisfying

$$
\sum_{k=1}^{n} v_{k} \leqq M n \text { for all } n \text { and some constant } M .
$$

Instead of $\left(g^{*}(\theta)\right)^{2}$, as defined by (13.4), form the series

$$
\sum_{n=1}^{\infty} \frac{\left|S_{n}^{\prime}(\theta)\right|^{2}}{n^{3}}-v_{n}
$$

\section{Corollary 2.}

$$
\left\|\left\{\Sigma \frac{\left|S_{n}^{\prime}(\theta)\right|^{2}}{n^{3}} v_{n}\right\}^{1 / 2}\right\|_{p} \leqq A_{p} M\|f\|_{p}, \quad \frac{2 \lambda+1}{\lambda+1}<p<\frac{2 \lambda+1}{\lambda}
$$

The constant $A_{p}$ does not depend on the sequence $\left\{v_{k}\right\}$ or $f$.

The proof is sufficiently similar to that of the theorem so that we shall omit it. See also [26, Chapter 15].

As a special case we can take

$$
\begin{aligned}
v_{n} & =\frac{n^{3}}{(n+1)^{2}} & & \text { if } n=2^{k} \\
& =0 & & \text { otherwise. }
\end{aligned}
$$

Then since $(n+1)\left[S_{n}(\theta)-\sigma_{n}(\theta)\right]=S_{n}^{\prime}(\theta)$, where $\sigma_{n}(\theta)$ are the Cesàro means (13.8) becomes

$$
\sum_{k=0}^{\infty}\left|S_{2}^{k}(\theta)-\sigma_{2}^{k}(\theta)\right|^{2}
$$


14. Multiplier theorem and behavior of lacunary partial sums. We come now to the main results of this part, which we formulate as two separate theorems. The first is an extension of the Marcinkiewicz multiplier theorem and includes the Littlewood-Paley theorem of decomposition into dyadic blocks. It may be formulated as follows:

Let $\mu_{0}, \mu_{1}, \cdots, \mu_{n}, \cdots$ be a sequence of constants (multipliers) and consider the linear transformation $T$, defined at least formally by

$$
T f \sim \sum_{n=1}^{\infty} a_{n} \mu_{n} P_{n}^{\lambda}(\cos \theta) \quad \text { whenever } f \sim \sum_{n=1}^{\infty} a_{n} P_{n}^{\lambda}(\cos \theta) .
$$

What is required are conditions on the sequence $\left\{\mu_{n}\right\}$ which will guarantee that the transformation $T$ is well defined and is continuous on $L^{p}$, that is

$$
\|T f\|_{p} \leqq A\|f\|_{p} .
$$

It is easy to see that when $p=2$, a necessary and sufficient condition of the sequence is the existence of a constant $M$, so that

$$
\left|\mu_{n}\right| \leqq M \text { for all } n
$$

We consider, in addition to this, the condition

$$
\sum_{k=1}^{n} k\left|\mu_{k}-\mu_{k+1}\right| \leqq M_{n} \quad \text { for all } n .
$$

The result is then

THEOREM 10. Suppose the sequence $\left\{\mu_{n}\right\}$ satisfies the conditions (i) and (ii) above. Then the transformation T, defined by (14.1) on all polynomials satisfies

$$
\|T f\|_{p} \leqq A_{p} M\|f\|_{p}, \quad \frac{2 \lambda+1}{\lambda+1}<p<\frac{2 \lambda+1}{\lambda} .
$$

Thus $T$ has a unique extension to all of $L^{p}$, which again satisfies (14.1) and (14.2).

REMARK. It should be noticed that the condition (ii) is equivalent with the condition

$$
\sum_{k=2^{n}}^{2 n+1}\left|u_{k}-u_{k+1}\right| \leqq M^{\prime} \text { for all } n
$$

This second form is the way such conditions are usually stated.

Proof. The main difficulty is the proof of inequality (14.2) for polynomials. The rest follows with easily managed technicalities. We begin by making the trivial simplifying assumption that $a_{0}=0$. 
Let us set $F=T f$. Then in view of Corollary 1 of Theorem 9, it is enough to prove

$$
\left\|g^{*}(\theta, F)\right\|_{p} \leqq A_{p}^{\prime} M\|f\|_{p}, \quad \frac{2 \lambda+1}{\lambda+1}<p<\frac{2 \lambda+1}{\lambda}
$$

Now

$$
S_{n}^{\prime}(\theta, F)=\sum_{k=1}^{n} a_{k} k \mu_{k} P_{k}^{\lambda}(\cos \theta)=\sum_{k=1}^{n-1} S_{k}^{\prime}(\theta, f)\left[\mu_{k}-\mu_{k+1}\right]+S_{n}^{\prime}(\theta, f) \mu_{n} .
$$

Therefore by Schwarz's inequality, and conditions (i) and (ii)

$$
\left|S_{n}^{\prime}(\theta, F)\right|^{2} \leqq 2 M n \sum_{k=1}^{n-1}\left|S_{k}^{\prime}(\theta, f)\right|^{2} \frac{\left|\mu_{k}-\mu_{k+1}\right|}{k}+2 M\left|S_{n}^{\prime}(\theta, f)\right|^{2} .
$$

Finally then,

$$
\left(g^{*}(\theta, F)\right)^{2}=\sum_{n=1}^{\infty} \frac{\left|S_{n}^{\prime}(\theta, F)\right|^{2}}{n^{3}} \leqq A M\left(\sum_{n=1}^{\infty} \frac{\left|S_{n}^{\prime}(\theta, f)\right|^{2} v_{n}}{n^{3}}+\sum_{n=1}^{\infty} \frac{\left|S_{n}^{\prime}(\theta, f)\right|^{2}}{n^{3}}\right),
$$

where $A$ is an absolute constant and $v_{n}=n\left|\mu_{n}-\mu_{n+1}\right|$.

Thus $\left\{v_{n}\right\}$ satisfies the condition (13.7) and Theorem 9 and its second corollary then show that

$$
\left\|g^{*}(\theta, F)\right\|_{2} \leqq A_{p} M\|f\|_{p} .
$$

The extension to the case where $f$ is an arbitrary $L^{p}$ function follows by straightforward limiting arguments.

That the usual decomposition theorem into dyadic blocks is contained in Theorem 10 can be seen as follows. For this we apply standard arguments related to the Rademacher functions $\phi_{0}(t), \phi_{1}(t), \cdots, \phi_{n}(t), \cdots$ defined on $(0,1)$. These functions are orthonormal and independent, and take on only the values \pm 1 . For each $t \in(0,1)$ define the linear operator $T_{t}$ by

$$
T_{t} f \sim \sum_{n=0}^{\infty} \phi_{n}(t) \sum_{k=2^{n}}^{2 n+1} a_{k} P_{k}^{\lambda}(\cos \theta)
$$

Then the sequence of multipliers $\mu_{n}$, so obtained, satisfy the conditions (i) and (ii) with $M=1$.

Hence we have $\left\|T_{t} f\right\|_{p} \leqq A_{p}\|f\|_{p}$. If we assume that $a_{0}=0$, and notice that $T_{t}^{2}=I$, we then have

$$
A_{p}^{-1}\|f\|_{p} \leqq\left\|T_{t} f\right\|_{p} \leqq A_{p}\|f\|_{p}, \quad \frac{2 \lambda+1}{\lambda+1}<p<\frac{2 \lambda+1}{\lambda} .
$$

If we integrate this with respect to $t$, we get (see [26, Chapter 15], for similar arguments) 
where

$$
A_{p}^{\prime-1}\|f\|_{p} \leqq\|\Delta(f)\|_{p} \leqq A_{p}^{\prime}\|f\|_{p}
$$

$$
\Delta(f)^{2}=\sum_{n=0}^{\infty}\left|\sum_{k=2^{n}}^{2 n+1-1} a_{k} P_{k}^{\lambda}(\cos \theta)\right|^{2} .
$$

We come now to the second result which deals with lacunary partial sums. In fact let $n_{1}, n_{2}, \cdots, n_{k}, \cdots$ be a lacunary sequence of positive integers, corresponding to Hadamard gaps, and described by the following condition: There exists a $\sigma>1$, so that $n_{k+1} / n_{k} \geqq \sigma>1$. The extension of the Littlewood-Paley theorem for the trigonometric case is then as follows.

THEOREM 11. Let $f(\theta) \in L^{p},(2 \lambda+1) /(\lambda+1)<p<(2 \lambda+1) / \lambda$. Then

(i) $\left\|\sup _{k}\left|S_{n_{k}}(\theta, f)\right|\right\|_{p} \leqq A_{p}\|f\|_{p}$,

(ii) $\left.\lim _{k \rightarrow \infty} S_{n_{k}}(\theta, f)\right)=f(\theta)$, almost everywhere and in the $L^{p}$-norm.

Proof. The key conclusion is (i); (ii) follows from it by straightforward arguments. In proving (i) we first deduce a maximal inequality for Cesàro means.

Thus let $\sigma_{n}(\theta)=\sigma_{n}(\theta, f)=(n+1)^{-1}\left(S_{0}(\theta, f)+\cdots+S_{n}(\theta, f)\right)$. We shall prove that

$$
\left\|\sup _{n}\left|\sigma_{n}(\theta, f)\right|\right\|_{p} \leqq A_{p}\|f\|_{p}, \quad \frac{2 \lambda+1}{\lambda+1}<p<\frac{2 \lambda+1}{\lambda} .
$$

In fact, we shall deduce (14.4) from its Abelian analogue, Theorem 2(d), in $\S 6$; the Tauberian condition will turn out to be, in effect, (13.6).

Let us temporarily use the following notation: $\sum_{k=0}^{\infty} u_{k}$ will be a numerical series, and we shall set

$$
S_{n}=\sum_{k=0}^{n} u_{k}, \quad \sigma_{n}=(n+1)^{-1} \sum_{k=0}^{n} S_{k}, \quad f(r)=\sum_{k=0}^{\infty} u_{k} r^{k} .
$$

Ultimately we shall make $u_{k}=a_{k} P_{k}^{\lambda}(\cos \theta)$, and therefore $S_{n}$ will be $S_{n}(\theta, f)$, $f(r)=f(r, \theta), \cdots$, etc.

We shall also have need of the expression

$$
\tau=\left\{\sum_{k=0}^{\infty}(k+1)\left|\sigma_{k}-\sigma_{k-1}\right|^{2}\right\}^{1 / 2}, \sigma_{-1}=0 .
$$

By summation by parts we have

$$
f(r)=\sum u_{k} r^{k}=(1-r) \sum S_{k} r^{k}=(1-r)^{2} \sum \sigma_{k}(k+1) r^{k}
$$

If we apply differences to the $\sigma_{k}$ and sum by parts again we get

$$
f(r)=(1-r) \sum_{k=0}^{\infty}\left(\sigma_{k}-\sigma_{k+1}\right)(k+1) r^{k}+\sum_{k=0}^{\infty}\left(\sigma_{k}-\sigma_{k-1}\right) r^{k+1} .
$$


Therefore

$$
\begin{aligned}
f(r)-\sigma_{n}=(1 & -r) \sum_{k=0}^{\infty}\left(\sigma_{k}-\sigma_{k-1}\right)(k+1) r^{k}+\sum_{k=0}^{n}\left(\sigma_{k}-\sigma_{k-1}\right)\left(r^{k+1}-1\right) \\
& +\sum_{n-1}^{\infty}\left(\sigma_{k}-\sigma_{k-1}\right) r^{k+1}
\end{aligned}
$$

By Schwarz's inequality, the fact that $1-r^{k+1} \leqq(k+2)(1-r)(0 \leqq r \leqq 1)$, it follows that

$$
\left.\left|f(r)-\sigma_{n}\right| \leqq \tau\left\{(1-r)\left(\sum_{k=0}^{\infty}(k+1) r^{2 k}\right)^{1 / 2}+(1-r)\left(\sum_{k=0}^{n} \frac{(k+2)^{2}}{k+1}\right)^{1 / 2}+\sum_{k=n+1}^{\infty} \frac{r^{2 k+2}}{k+1}\right)^{1 / 2}\right\} .
$$

If we set $r=1-1 / n$, the terms within the brackets are all bounded. Hence

$$
\sup \left|\sigma_{n}\right| \leqq \sup _{0 \leqq r<1}|f(r)|+A \tau .
$$

If we particularize to $u_{k}=a_{k} P_{k}^{\lambda}(\cos \theta)$, we get

$$
\sup _{n}\left|\sigma_{n}(\theta, f)\right| \leqq \sup _{0 \leqq r<1}|f(r, \theta)|+A\left\{\sum_{k=0}^{\infty}(k+1)\left|\sigma_{k}(\theta, f)-\sigma_{k-1}(\theta, f)\right|^{2}\right\}^{1 / 2},
$$

where $f(r, \theta)$ is the Poisson integral of $f(\theta)$,

$$
f(r, \theta)=\sum_{k=0}^{\infty} a_{k} r^{k} P_{k}^{\lambda}(\cos \theta) \text {. }
$$

Next we notice that $\sigma_{n}(\theta, f)-\sigma_{n-1}(\theta, f)=(n(n+1))^{-1} S_{n}^{\prime}(\theta, f)$. Therefore

$$
\sum_{k=1}^{\infty}(k+1)\left|\sigma_{k}(\theta, f)-\sigma_{k-1}(\theta, f)\right|^{2}=\sum_{k=1}^{\infty} k^{-2}\left(k^{-1}+1\right)^{-1}\left|S_{k}^{\prime}(\theta, f)\right|^{2} \leqq\left(g^{*}(\theta, f)\right)^{2} .
$$

Since we have taken $a_{0}=0$, then $\sigma_{0}=0$, and therefore

$$
\sup _{n}\left|\sigma_{n}(\theta, f)\right| \leqq \sup _{0 \leqq r<1}|f(r, \theta)|+A g^{*}(\theta, f) \text {. }
$$

A combination of Theorem 2(d) of $\S 6$ ) and Corollary 1 to Theorem 9 (in $\S 13$ then gives (14.4).

Next

$$
\sup _{n_{k}}\left|S_{n_{k}}(\theta, f)\right| \leqq \sup _{n_{k}}\left|\sigma_{n_{k}}(\theta, f)\right|+\sup _{n_{k}}\left|S_{n_{k}}(\theta, f)-\sigma_{n_{k}}(\theta, f)\right|
$$

Thus

(14.6) $\sup _{n_{k}}\left|S_{n_{k}}(\theta, f)\right| \leqq \sup _{n_{k}}\left|\sigma_{n_{k}}(\theta, f)\right|+\left(\sum_{n_{k}}\left|S_{n_{k}}(\theta, f)-\sigma_{n_{k}}(\theta, f)\right|^{2}\right)^{1 / 2}$. 
However once the fixed lacunary sequence $\left\{n_{k}\right\}$ has been chosen then we have

$$
\left\|\left(\sum_{n_{k}}\left|S_{n_{k}}(\theta, f)-\sigma_{n_{k}}(\theta, f)\right|^{2}\right)^{1 / 2}\right\|_{p} \leqq A_{p}\|f(\theta)\|_{p} .
$$

In fact the special case of (14.7) is then $n_{k}=2^{k}$, obtained in the remarks following Corollary 2 to Theorem 9. The general case of $\left\{n_{k}\right\}$ considered is then treated in exactly the same way.

(14.4), (14.5) and (14.7) immediately imply conclusion (i) of the theorem. As we have stated before, (ii) then follows from (i) by well-known arguments.

\section{Chapter III}

15. Convolution structure and fractional integration. Up to the present our main emphasis has been to associate to each development its appropriate "harmonic" (and "conjugate harmonic") functions. This is seen most explicitly in Chapter I ( $\$ \S 2-9)$; to a large degree this is also the case for Chapter II, in view of the definition of the $g$-function which plays a key role there.

We now shift our point of view and study a convolution structure naturally associated with ultraspherical (and many other) expansions. Such convolution structures have been studied for some time by several authors, but the more precise knowledge in the case of ultraspherical developments appears first in Bochner [4].

We begin first by giving a definition of a convolution structure, and then proving a general theorem of "fractional integration" for such a structure, and finally we shall apply this in the case of ultraspherical expansions.

The resulting theorem can be stated as follows:

THEOREM 12. Define the operator $I_{\alpha}, 0<\alpha$, as follows: If

$$
f \sim \sum_{n=0}^{\infty} a_{n} P_{n}^{\lambda}(\cos \theta)
$$

then

$$
I_{\alpha}(f) \sim \sum_{n=1}^{\infty} n^{-\alpha} a_{n} P_{n}^{\lambda}(\cos \theta)
$$

Then

$$
\left\|I_{\alpha}(f)\right\|_{r} \leqq A_{r, p}\|f\|_{p}
$$

whenever $1<p<\infty, 1<r<\infty$, and $1 / r=1 / p-\alpha /(2 \lambda+1)$.

We must study first, however, some general properties of convolution structures.

Let $M$ be a measure space with measure $d m$. We define the $L^{p}$-spaces and their norms, $\|\cdot\|_{\nu}$, in the usual fashion. We say we have a convolution structure 
on $M$ if we have a bilinear mapping of $L^{1} \times L^{1}$ to $L^{1}$ given by $(f, g) \rightarrow f * g$, which satisfies the following properties:

$$
\begin{aligned}
\|f * g\|_{1} & \leqq\|f\|_{1}\|g\|_{1}, \\
\|f * g\|_{\infty} & \leqq\|f\|_{1}\|g\|_{\infty}, \text { if } g \in L^{1} \cap L^{\infty}, \\
\|f * g\|_{\infty} & \leqq f\left\|_{\infty}\right\| g \|_{1}, \quad \text { if } f \in L^{1} \cap L^{\infty} .
\end{aligned}
$$

It should be noted that we have not assumed that $f * g=g * f$-that is, we shall not need commutativity of the convolution product. An example of the above structure is the usual convolution on any locally compact (unimodular) groupand more generally on various homogeneous spaces of such groups.

We observe first Young's inequality for convolution structures, which we state as a lemma.

LeMmA 10 . Let $1 \leqq p, q, r \leqq \infty$, with $1 / r=1 / q+1 / p-1$. Then

$$
\|f * g\|_{r} \leqq\|f\|_{p}\|g\|_{q}
$$

if

$$
f \in L^{1} \cap L^{p} \text { and } g \in L^{1} \cap L^{g} .
$$

Proof. It suffices to prove (15.1) when both $f$ and $g$ are simple functions (that is, finite linear combinations of characteristic functions of sets of finite measure). From this special case the more general one stated then follows by a simple limiting argument. In fact, once (15.1) has been proved a similar argument shows that the convolution has a natural (unique) extension to a bilinear mapping on all of $L^{p} \times L^{q}$ to $L^{r}$, satisfying (15.1), whenever $p, q$, and $r$ satisfy the conditions of the lemma.

However (15.1), for simple functions, follows in a straightforward fashion from a two-fold application of the bilinear Riesz-Thorin convexity theorem in conjunction with conditions (1), (2) and (3) above. (See Zygmund [26, Vol. II, p. 106].)

We come now to fractional integration. It was observed by $R$. O'Neil that the classical (Euclidean $n$-space) fractional integration theorem can be restated as an inequality like (15.1), but where the $L^{q}$-norm of $g$ is replaced by a weaker norm-the so-called "weak-type" norm.

For our purposes, the weak-type norm of exponent $q,\|\cdot\|_{q}^{*}$, can be defined as follows

$$
\|g\|_{q}^{*}=\sup _{E} \frac{\int|g| \chi_{E} d m}{\left\|\chi_{E}\right\|_{q^{\prime}}} .
$$

The sup is taken over all sets $E$ of finite positive measure, $\chi_{E}$ denoting the characteristic function of the set $E$, and $q^{\prime}$ denoting the conjugate exponent to $q, 1 / q^{\prime}+1 / q=1$. 
We observe that

$$
\|g\|_{q}^{*} \leqq\|g\|_{q},
$$

and $\|\cdot\|_{q}^{*}$ has the properties of a norm: $\left\|c_{1} g_{1}+c_{2} g_{2}\right\|_{q}^{*} \leqq\left|c_{1}\right|\left\|g_{1}\right\|_{q}^{*}$ $+\left|c_{2}\right|\left\|g_{2}\right\|_{q}^{*}$, for constants $c_{1}$ and $c_{2}$; and $\|g\|_{q}^{*}=0$, only if $g=0$. It should also be noted that $\|g\|_{q}^{*}=\|g\|_{q}$, when $q=1$ or $\infty$; and for all $q, 1 \leqq q \leqq \infty$, when $g$ is the characteristic function of a set. In general, however, $\|g\|_{q}^{*}$ is smaller than $\|g\|_{q}$.

With those definitions we can now state the theorem of fractional integration for convolution structures $\left({ }^{8}\right)$.

THEOREM $13\left({ }^{9}\right)$.

$$
\left\|f^{*} g\right\|_{r} \leqq A_{p, q}\|f\|_{p}\|g\|_{q}^{*}
$$

if $1<p, q, r<\infty$ and $1 / r=1 / q+1 / p-1$.

Proof. Let us fix $g$ with $\|g\|_{q}^{*}=1$, and consider the linear transformation $T: f \rightarrow f * g$. We remark first that $T(f)$ is a well-defined function (in the space $\left.L^{p}+L^{\infty}\right)$. In fact write $g=g^{1}+g_{1}$, where $g^{1}=g$ if $|g|>1$ and $g^{1}=0$ otherwise; $g_{1}=g$ if $|g| \leqq 1, g_{1}=0$ otherwise. Notice first that $g^{1} \in L^{1}$; also the conditions on $p, q, r$ imply that $q<p^{\prime},\left(1 / p+1 / p^{\prime}=1\right)$. From this it is easily follows that $g_{1} \in L^{p^{\prime}}$. Now $T f=f * g$ will be defined $T f=T_{1} f+T^{1} f$ where $T_{1} f * g_{1}=f * g_{1}, T^{1} f=f * g^{1}$. Then $T_{1} f \in L^{\infty}$, and $T^{1} f \in L^{p}$ (by Lemma 10). It is to be noted that the definition of $T f$ actually does not depend on the particular splitting used of $g$ into a function of $L^{1}$ and $L^{p^{\prime}}$.

In proving (15.3) we shall prove first the weaker statement

$$
\|T f\|_{r}^{*} \leqq A_{p, q}\|f\|_{p} \quad(T f=f * g)
$$

with $1<p, q, r<\infty$ and $1 / r=1 / p+1 / q-1$.

To do this we shall repeat in a more precise way the argument which showed that $T f$ was well defined, paying attention to the all-important details.

Thus we write $T f=T^{\alpha} f+T_{\alpha} f=f^{*} g^{\alpha}+f^{*} g_{\alpha}$, where $g^{\alpha}=g$ if $|g|>\alpha$, while $g_{\alpha}=g$ if $|g| \leqq \alpha . \alpha$ is positive and will be fixed momentarily.

We begin by computing $\left\|g^{\alpha}\right\|_{1}$ and $\left\|g_{\alpha}\right\|_{p^{\prime}}$. However, $\left\|g_{\alpha}\right\|_{1}=\int_{E}|g| d x$ $\leqq\left(m\left(E^{\alpha}\right)\right)^{1-1 / q}$, where $E^{\alpha}=\{x|| g(x) \mid>\alpha\}$, since $\|g\|_{q}^{*} \leqq 1$. But $\alpha<|g(x)|$ on $E^{\alpha} ;$ and thus

$$
\alpha m\left(E^{\alpha}\right)<\int_{E}|g| d x \leqq m\left(E^{\alpha}\right)^{1-1 / q} .
$$

(8) In the case of ordinary convolution on a group the theorem was first proved by O'Neil. See his paper [12].

(9) The precise meaning of $f * g$ is discussed in the proof of the theorem. 
Combining the extreme terms given $m\left(E^{\alpha}\right) \leqq \alpha^{-q}$, and therefore

$$
\left\|g^{\alpha}\right\|_{1} \leqq \alpha^{-q+1} \text { if }\|g\|_{q}^{*} \leqq 1 .
$$

Next, if $\lambda(\beta)=m\left(E^{\beta}\right)$, then

$$
\left\|g_{\alpha}\right\|_{p^{\prime}}^{p^{\prime}}=-\int_{0}^{\alpha} \beta^{p^{\prime}} d \lambda(\beta)=p^{\prime} \int_{0}^{\alpha} \beta^{p^{\prime}-1} \lambda(\beta) d \beta .
$$

We have already seen that $m\left(E^{\alpha}\right) \leqq \alpha^{-q}$ for all $\alpha>0$; hence $\lambda(\beta) \leqq \beta^{-q}$ This gives

Finally

$$
\left\|g_{\alpha}\right\|_{p^{\prime}}^{p^{\prime}} \leqq p^{\prime} \int_{0}^{\alpha} \beta^{p^{\prime}-1-q} d \beta=\frac{p^{\prime}}{p^{\prime}-q} \alpha^{p^{\prime}-q}
$$

$$
\left\|g_{\alpha}\right\|_{p^{\prime}} \leqq\left(\frac{p^{\prime}}{p^{\prime}-q}\right)^{1 / p^{\prime}} \|^{1-q / p^{\prime}}=c \alpha^{1-q / p^{\prime}}
$$

Now

$$
\begin{aligned}
\int_{E}\left|T^{\alpha} f\right| d x & \leqq\left\|T^{\alpha} f\right\|_{p}(m(E))^{1-1 / p}=\left\|f^{*} g^{\alpha}\right\|_{p}(m(E))^{1-1 / p} \\
& \leqq\|f\|_{p}\left\|g^{\alpha}\right\|_{1} m(E)^{1-1 / p}\|f\|_{p} \alpha^{-q} m(E)^{1-1 / p},
\end{aligned}
$$

by (15.5) and Lemma 10 again.

Now choose $\alpha$ by setting

$$
\alpha^{-q+1} m(E)^{1-1 / p}=\alpha^{1-q / p^{\prime}} m(E) .
$$

This determines $\alpha$ as $\alpha^{-q}=m(E)$ and gives

$$
\alpha^{-q+1} m(E)^{1-1 / p}=\alpha^{1-q / p^{\prime}} m(E)=m(E)^{1-1 / r},
$$

where $1 / r=1 / p+1 / q-1$.

Hence we have

$$
\begin{aligned}
\int_{E}|T f| d x & \leqq \int_{E}\left|T^{\alpha} f\right| d x+\int_{E}\left|T_{\alpha} f\right| d x \\
& \leqq(c+1) m(E)^{1-1 / r}\|f\|_{p} .
\end{aligned}
$$

If we take the sup over the sets $E$ we obtain

$$
\|T f\|_{r}^{*} \leqq(c+1)\|f\|_{p},
$$

and this is (15.3).

Let us now interpret the condition $\|g\|_{r}^{*} \leqq A$, for a general function $g$. In order to do this consider the distribution function $\lambda$ of $|g|$. That is, $\lambda(\alpha)=m\left(E^{\alpha}\right)=m\{x:|g(x)|>\alpha\}$. 
Now

$$
\alpha \lambda(\alpha) \leqq \int_{E_{\alpha}}|g| d m \leqq A m\left(E^{\alpha}\right)^{1-1 / r}=A(\lambda(\alpha))^{1-1 / r}
$$

Therefore, $\lambda(\alpha) \leqq A^{r} \alpha^{-r}, 0<\alpha<\infty$.

This shows that the inequality (15.3) implies

$$
m\{x:|T f|>\alpha\} \leqq\left(\frac{A_{p, q}\|f\|_{p}}{\alpha}\right)^{r}, 1<p, q, r<\infty, 1 / r=1 / p+1 / q-1 .
$$

For fixed $p, q, r,(15.4)$ is exactly the statement that the mapping $f \rightarrow T f$ is of weak type $(p, r)$, in the sense of the Marcinkiewicz interpolation theorem; see [26, Vol. II, p. 111]. Applying this theorem then gives

$$
\|T f\|_{r}=\|f * g\|_{r} \leqq A_{p, q}^{\prime}\|f\|_{p}, \quad 1<p, q, r<\infty, 1 / r=1 / p+1 / q-1 .
$$

Finally, if we drop the normalization $\|g\|_{q}^{*}=1$, we get (15.2) and the theorem. We now come to the convolution structure associated with ultraspherical expansions.

It will be convenient to change our notation slightly by renormalizing the ultraspherical polynomials and the underlying measure of our space. We fix $\lambda>0$, throughout the discussion.

Thus set $P_{n}^{*}(\cos \theta)=P_{n}^{\lambda}(\cos \theta) / P_{n}^{\lambda}(1)$, and hence $P_{n}^{*}(1)=1$. We shall also set $\|f\|_{p}=\left(c_{\lambda}^{-1} \int_{0}^{\pi}|f(\theta)|^{p} \sin ^{2 \lambda-1} \theta d \theta\right)^{1 / p}$, where $c_{\lambda}=\int_{0}^{\pi} \sin ^{2 \lambda-1} \theta d \theta$. The normalizing constants $\rho_{n}$ are defined by

Thus

$$
\rho_{n}^{-1}=\left\|P_{n}^{*}\right\|_{2}
$$

$$
\rho_{n}=\frac{\lambda+n}{\lambda} \frac{\Gamma(n+2 \lambda)}{n ! \Gamma(2 \lambda)}
$$

(see [4, p. 25]). If $f(\theta) \sim \sum a_{n} P_{n}^{\lambda}(\cos \theta)$ we also set

$$
f(\theta) \sim \sum a_{n}^{*} \rho_{n} P_{n}^{*}(\cos \theta) .
$$

We now define $f * g$ by

$$
(f * g)(\theta)=c_{\lambda}^{-2} \int_{0}^{\pi} \int_{0}^{\pi} f(\phi) g(\psi) \sin ^{2 \lambda-1} \phi \sin ^{2 \lambda-1} t d \phi d t,
$$

where

$$
\cos \psi=\cos \theta \cos \phi+\sin \theta \sin \phi \cos t
$$

The crucial properties of this convolution product are two-fold.

$$
\begin{gathered}
|f * g| \leqq|f| *|g| . \\
P_{n}^{*} * P_{m}^{*}=\rho_{n}^{-1} \delta_{n, m} P_{n}^{*} .
\end{gathered}
$$


The first is obvious, and the second is in [4, p. 29].

From (15.10) it follows that if

$$
f \sim \sum a_{n}^{*} \rho_{n} P_{n}^{*} \quad \text { and } \quad g \sim \sum b_{n}^{*} \rho P_{n}^{*}
$$

then

$$
f * g \sim \sum a_{n}^{*} b_{n}^{*} \rho_{n} P_{n}^{*}
$$

which is the multiplicative property of convolutions. From (15.9), (15.10), and (15.11) it follows easily that $f * g=g * f$, and since $f * 1=\|f\|_{1} \leqq\|f\|_{\infty}$ we get $\|f * g\|_{\infty}=\|g * f\|_{\infty} \leqq\|f\|_{1}\|g\|_{\infty}$.

Also if $f$ and $g$ are non-negative

$$
\|f * g\|_{1}=c_{\lambda}^{-1} \int_{0}^{\pi}(f * g)(\theta) \sin ^{2 \lambda-1} \theta d \theta=\|f\|_{1}\|g\|_{1} .
$$

Thus, in general,

$$
\|f * g\|_{1} \leqq\|f\|_{1}\|g\|_{1}
$$

We have therefore verified the basic properties of the convolution structure.

We now come to the proof of Theorem 12 stated earlier in this section. It can be restated as follows:

If

$$
f \sim \sum_{n=0}^{\infty} a_{n}^{*} \rho_{n} P_{n}^{*}(\cos \theta)
$$

and

$$
I_{\alpha}(f) \sim \sum_{n=1}^{\infty} a_{n}^{*} \rho_{n} n^{-\alpha} P_{n}^{*}(\cos \theta)
$$

then

$$
\left\|I_{\alpha}(f)\right\|_{r} \leqq A_{r, p}\|f\|_{p},
$$

whenever

$$
1<p<\infty, 1<r<\infty \text { and } 1 / r=1 / p-\alpha /(2 \lambda+1) \text {. }
$$

However,

$$
I_{\alpha}(f)=f * K_{\alpha}
$$

where

$$
K_{\alpha}(\theta) \sim \sum_{n=1}^{\infty} n^{-\alpha} \rho_{n} P_{n}^{*}(\cos \theta) .
$$

Therefore, in view of Theorem 13, it suffices to prove that

$$
\left\|K_{\alpha}\right\|_{q}^{*} \leqq A_{q, \alpha}<\infty \text { for } 1 / q-1=-\alpha /(2 \lambda+1),
$$

where $\|\cdot\|_{q}^{*}$ is the weak-type norm. 
Let us set $x=\cos \theta$, and $K_{\alpha}(\theta)=k_{\alpha}(x)$. Then as is known, (see [4, p. 25]),

$$
\frac{1-r^{2}}{\left(1-2 r x+r^{2}\right)^{\lambda+1}}=\sum_{n=0}^{\infty} \rho_{n} r^{n} P_{n}^{*}(x), \quad 0 \leqq r<1 \text {. }
$$

Thus

$$
k_{\alpha}(x)=\frac{1}{\Gamma(\alpha)} \int_{0}^{1}\left[\frac{1-r^{2}}{\left(1-2 r x+r^{2}\right)^{2}+1}-1\right](\log 1 / r)^{\alpha-1} \frac{d r}{r}, \quad \alpha>0 .
$$

We break up this integral into two parts: from 0 to $1 / 2$ and $1 / 2$ to 1 . We notice without difficulty that the first integral is uniformly bounded in $x$. We now consider the second integral, dropping first the factor $\Gamma(\alpha)^{-1}$, and then the constant $\int_{1 / 2}^{1}(\log 1 / r)^{\alpha-1} r^{-1} d r$. What remains is bounded by a constant multiple of

$$
\int_{1 / 2}^{1} \frac{1-r^{2}}{\left(1-2 r x+r^{2}\right)^{\lambda+1}}(1-r)^{\alpha-1} d r .
$$

We remark that here we may assume that say $3 / 4 \leqq x<1$, since in the interval $-1 \leqq x \leqq 3 / 4$ this integral is uniformly bounded. We now decompose the integral in (15.12) as a sum of an integral where $(1-r)^{2} \geqq 1-x$, and another where $(1-r)^{2}<1-x$.

Now,

$$
1-2 r x+r^{2}=(1-r)^{2}+2 r(1-x)
$$

Thus

$$
\left(1-2 r x+r^{2}\right)^{\lambda+1} \geqq(1-r)^{2 \lambda+2} \text { when }(1-r)^{2} \geqq(1-x)
$$

and

$$
\left(1-2 r x+r^{2}\right)^{\lambda+1} \geqq(1-x)^{\lambda+1} \text { when }(1-r)^{2}<(1-x)(r \geqq 1 / 2) .
$$

Combining the above we see that (15.12) is majorized by twice

$$
\int_{1 / 2}^{1-(1-x)^{1 / 2}}(1-r)^{\alpha-2 \lambda-2} d r+(1-x)^{-\lambda-1} \int_{1-(1-x)^{1 / 2}}^{1}(1-r)^{\alpha} d r
$$

Altogether, this is bounded by $A(1-x)^{\alpha / 2-\lambda-1 / 2}$. Thus

$\left|k_{\alpha}(x)\right|=\left|K_{\alpha}(\cos \theta)\right| \leqq A(1-x)^{\alpha / 2-\lambda-1 / 2}+A \leqq A^{\prime}(1-x)^{\alpha / 2-\lambda-1 / 2}=A(\sin \theta / 2)^{\alpha-2 \lambda-1}$, since $\alpha<2 \lambda+1, x=\cos \theta$.

Finally it suffices to show that

$$
\left\|(\sin \theta / 2)^{\pi-2 \lambda-1}\right\|_{q}^{*}<\infty, \quad 1-1 / q=\frac{\alpha}{2 \lambda+1},
$$

or what is the same 


$$
\sup _{E} \frac{\int_{E}(\sin \theta / 2)^{\alpha-2 \lambda-1} \sin ^{2 \lambda} \theta d \theta}{\left(\int_{E} \sin ^{2 \lambda} \theta d \theta\right)^{1-1 / q}}<\infty
$$

Since $\alpha-2 \lambda-1<0$, the function $(\sin \theta / 2)^{\alpha-2 \lambda-1}$ is decreasing on $(0, \pi)$. Thus the above supremum is equal to

$$
\sup _{0<l \leqq \pi} \frac{\int_{0}^{l}(\sin \theta / 2)^{\alpha-2 \lambda-1} \sin ^{2 \lambda} \theta d \theta}{\left(\int_{0}^{l} \sin ^{2 \lambda} \theta d \theta\right)^{1-1 / q}} .
$$

But the last quotient is bounded by constant $\times \sup l^{\alpha} / l^{(2 \lambda+1)(1-1 / q)}$.

However, $\alpha /(2 \lambda+1)=1-1 / q$, which proves the assertion, and hence the theorem.

16. Fourier-Bessel transforms (Hankel transforms). We shall now discuss the analogue of our study of ultraspherical expansions which leads to Bessel function and the Fourier-Bessel (or Hankel) transforms.

The wide-ranging analogy between (discrete) ultraspherical expansions and (continuous) Fourier-Bessel expansions will be used in what follows below. The analogy holds not only for the statements of the theorems but carries over also to the details of their proofs. Two slight qualifications must be added to this assertion. First, various formulae and estimates take a somewhat simpler form in the Fourier-Bessel case than the ultraspherical case. Second, the ultraspherical case, as opposed to the Fourier-Bessel transform case, deals with a compact domain as contrasted to a noncompact domain. Thus certain additional arguments are needed in problems of "harmonic majorization"' (see Lemma 11 below).

However, in the main, the analogy between these two cases is so close that it is not necessary to duplicate most of the proofs in the second case. For this reason, and to save space, our discussion will be brief, omitting proofs in every case (except for Lemma 11).

Our starting point is, as indicated in the Introduction, functions in Euclidean $n$-space which are radial. Thus let $\bar{y}=\left(y_{1}, \cdots, y_{n}\right) \in E_{n}$, and let $\bar{f}(\bar{y})=f\left(y_{1}, \cdots, y_{n}\right)$ be a radial function, i.e., $\bar{f}(\bar{y})=f(y)$, where $y=|\bar{y}|=\left(y_{1}^{2}+\cdots+y_{n}^{2}\right)^{1 / 2}$.

Consider the Fourier transform of such a function

Then

$$
\bar{f}^{\wedge}(\bar{\xi})=(2 \pi)^{-n / 2} \int_{E_{n}} \bar{f}(\bar{y}) e^{i \bar{\xi} \cdot \bar{y}} d \bar{y}
$$

$$
\hat{f}(\xi)=\int_{0}^{\infty} V_{(n-2) / 2}(\xi y) f(y) y^{n-1} d y
$$


with $\xi=|\xi|$ and

$$
V_{a}(t)=t^{-a} J_{a}(t)
$$

(See [5, Chapter II].)

If $\bar{u}(x, \bar{y})$ is the harmonic function in $x>0$, with boundary values $\bar{f}(\bar{y})$ (when $x=0)$, then this Poisson integral is given by

$$
\begin{aligned}
\bar{u}(x, \bar{y}) & =u(x, y)=\int_{0}^{\infty} e^{-x \xi} V_{(n-2) / 2}(\xi y) f(\xi) \xi^{n-1} d \xi \\
& =\int_{0}^{\infty} P(x, y, z) f(z) z^{n-1} d z,
\end{aligned}
$$

with

$$
P(x, y, z)=(y z)^{-(n-2) / 2} \int_{0}^{\infty} e^{-x \xi} J_{(n-2) / 2}(\xi y) J_{(n-2) / 2}(\xi z) \xi d \xi .
$$

Also $u(x, y)$ satisfies the radial "Laplace equation"

$$
\frac{\partial^{2} u}{\partial x^{2}}+\frac{\partial^{2} u}{\partial y^{2}}+\frac{n-1}{y} \frac{\partial u}{\partial y}=0
$$

Incidentally, the Plancherel formula in this case becomes

$$
\int_{0}^{\infty}|\hat{f}(\xi)|^{2} \xi^{n-1} d \xi=\int_{0}^{\infty}|f(y)|^{2} y^{n-1} d y .
$$

We now set $n-1=2 \lambda$, and we pass by analogy from half-integral $\lambda$ to general positive $\lambda$. In general, we have

$$
u(x, y)=\int_{0}^{\infty} P(x, y, z) f(z) z^{2 \lambda} d z,
$$

where

$$
P(x, y, z)=(y z)^{-i-1 / 2} \int_{0}^{\infty} e^{-x \xi} J_{\lambda-1 / 2}(y \xi) J_{\lambda-1 / 2}(z \xi) \xi d \xi .
$$

$u(x, y)$ now satisfies the equation

$$
\frac{\partial^{2} u}{\partial x^{2}}+\frac{\partial^{2} u}{\partial y^{2}}+\frac{2 \lambda}{y} \frac{\partial u}{\partial y}=0
$$

All our $L^{p}$-spaces will be taken with respect to the measure $y^{2 \lambda} d y$, so that

$$
\|f\|_{p}=\left(\int_{0}^{\infty}|f(y)|^{p} y^{2 \lambda} d y\right)^{1 / p} .
$$


In this way the mapping

$$
f(y) \rightarrow \hat{f}(\xi)=\int_{0}^{\infty} V_{\lambda-1 / 2}(\xi y) f(y) y^{2 \lambda} d y
$$

becomes a unitary transformation on $L^{2}$. (See [19, Chapter 8].)

$u(x, y)$ can be re-expressed in terms of this transformation:

$$
u(x, y)=\int_{0}^{\infty} e^{-x \xi} V_{\lambda-1 / 2}(\xi y) \hat{f}(\xi) \xi^{2 \lambda} d \xi .
$$

We return to the Poisson kernel $P(x, y, z)$, and its conjugate $Q(x, y, z)$ (to be defined later), and find explicit expressions for these.

For this purpose we consider the formula of Weinstein [23]

$$
\begin{aligned}
\phi_{b}(x, y) & =S_{p-1} \int_{0}^{\pi}\left(b^{2}+x^{2}+y^{2}-2 b y \cos a\right)^{-p / 2}(\sin a)^{p-1} d a \\
& =C_{q} b^{-q} y^{-q} \int_{0}^{\infty} e^{-x t} J_{q}(y t) J_{q}(b t) d t
\end{aligned}
$$

with

$$
S_{\rho-1}^{-1}=\pi^{1 / 2} \Gamma(p / 2)\left(\Gamma\left(\frac{p+1}{2}\right)\right)^{-1}, \quad C_{q}=2^{2 q} \Gamma^{2}(q+1)(\Gamma(2 q+1))^{-1},
$$

and $p=2 q+1 . \phi_{b}(x, y)$ also satisfies the "Laplace equation" (16.2) with $p=2 \lambda$. By differentiating with respect to $x$ we get

$$
P(x, y, b)=\frac{2 \lambda x}{\pi} \int_{0}^{\pi}\left(b^{2}+x^{2}+y^{2}-2 b y \cos a\right)^{-\lambda-1}(\sin a)^{2 \lambda-1} d a .
$$

Since we have $P(x, y, b)=-C_{q}^{-1} \partial\left(\phi_{b}(x, y)\right) / \partial x$, and $\phi$ satisfies (16.2), its conjugate should be given by $C_{q}^{-1} \partial\left(\phi_{b}(x, y)\right) / \partial y$.

We thus define $Q(x, y, b)$ by $Q(x, y, b)=C_{q}^{-1} \partial\left(\phi_{\dot{b}}\right) / \partial y$, and we get

$$
Q(x, y, b)=-(b y)^{\lambda-1 / 2} \int_{0}^{\infty} e^{-x t} J_{\lambda+1 / 2}(y t) J_{\lambda-1 / 2}(b t) t d t
$$

and

$$
Q(x, y, b)=-\frac{2 \lambda}{\pi} \int_{0}^{\pi} \frac{(y-b \cos \alpha) \cdot(\sin a)^{2 \lambda-1}}{\left(b^{2}+x^{2}+y^{2}-2 b y \cos a\right)^{\lambda+1}} d a .
$$

For an arbitrary $f$ (say $f \in L^{p}\left(y^{2 \lambda} d y\right)$ ), we have defined its Poisson integral $u(x, y)$ by (16.1) and $u$ satisfies (16.2). Define the conjugate $v(x, y)$ to $u(x, y)$ by

$$
v(x, y)=y^{2 \lambda} V(x, y)=y^{2 \lambda} \int_{0}^{\infty} Q(x, y, z) f(z) z^{2 \lambda} d z .
$$


Then it follows that $u$ and $v$ satisfy the "Cauchy-Riemann" equations:

$$
v_{y}=y^{2 \lambda} u_{x}, \quad v_{x}=-y^{2 \lambda} u_{y},
$$

or if $U(x, y)=u(x, y), V(x, y)=y^{-2 \lambda} v(x, y)$,

$$
V_{x}+U_{y}=0, \quad U_{x}-V_{y}-\frac{2 \lambda}{y} V=0 .
$$

We therefore see that the notion of conjugacy given by (16.6) and naturally associated to the Fourier-Bessel transform (16.3) is the same as the notion of conjugacy studied earlier and associated to ultraspherical expansions.

What distinguishes these cases are the domains of the $(x y)$-plane under consideration. In the ultraspherical case we had the upper semi-disc $x^{2}+y^{2}<1, y>0$ and since $U(x, y)$ was even in $y$ and $V(x, y)$ was odd in $y$, we ultimately considered the whole disc.

In the present case the domain is initially the quarter-plane $x>0, y>0$; again because the $U(x, y)$ and $V(x, y)$ which arise are respectively even and odd in $y$ we extend consideration to the whole half-plane $x>0$. In the case of FourierBessel series considered briefly in $\$ 18$ below the domain becomes the half-strip $0 \leqq x, 0 \leqq y \leqq 1$; and then because of evenness the doubled strip $0 \leqq x,-1 \leqq y \leqq 1$.

We shall now briefly indicate how the results of $\S \S 3$ to 15 can be reformulated and proved in variants appropriate for this setting $\left(1^{0}\right)$.

(a) Coming first to the maximum principle (Theorem 1 in $\$ 3$ ), we see that in view of (16.2) and what has been said above, this theorem is equally applicable to the present case. However, in the present case the domains in question are not bounded. It will be necessary, therefore, to supplement Theorem 1 with an additional lemma and its corollary.

LemMa 11. Suppose (1) $u(x, y)$ is continuous in $0 \leqq x<\infty,-\infty<y<\infty$, and even in $y$.

(2) In the region where $u(x, y)>0, u$ is of class $C^{2}$ and satisfies $\partial^{2} u / \partial x^{2}+\partial^{2} u / \partial y^{2}+2 \lambda y^{-1} \partial u / \partial y \geqq 0$, there.

(3) $u(0, y)=0$.

(4) For some $p, 1 \leqq p<\infty$,

$$
\sup _{0<x<\infty} \int_{0}^{\infty}|u(x, y)|^{p} y^{2 \lambda} d y \leqq M<\infty .
$$

Conclusion: $u(x, y) \leqq 0$.

COROLlaRY. If in addition $\partial^{2} y / \partial x^{2}+\partial^{2} y / \partial y^{2}+2 \lambda y^{-1} \partial u / \partial y=0$ everywhere then $u(x, y) \equiv 0$.

(10) Recall that we always have $\lambda \geqq 0$. 
Proof. Fix $0<\rho<\infty$, and define $f_{\rho}(\theta)$ by $f_{\rho}(\theta)=|u(\rho \cos \theta, \rho \sin \theta)|$ for $|\theta| \leqq \pi / 2$, and $f_{\rho}(\theta)=-f_{\rho}(\pi-\theta)$, for $(\pi / 2) \leqq \theta \leqq 3 \pi / 2$. Then $f_{\rho}(\theta)$ is continuous, since $f( \pm \pi / 2)=0$. Now

$$
\int_{0}^{R} \int_{0}^{\pi}|f(\theta)|^{p}(\sin \theta)^{2 \lambda} d \theta \rho^{2 \lambda+1} d \rho=\frac{1}{2} \int_{x^{2}+y^{2} \leqq R^{2}}|u(x, y)|^{p} y^{2 \lambda} d y d x \leqq M R,
$$

because of condition (4).

Thus if $J(\rho)=\int_{0}^{\pi}\left|f_{l}(\theta)\right|^{p}(\sin \theta)^{2 \lambda} d \theta$, then $\int_{0}^{R} J(\rho) \rho^{2 \lambda+1} d \rho \leqq M R$, and hence $\liminf _{\rho \rightarrow \infty} J(\rho) \rho^{2 \lambda+1} \leqq M$. In particular, there exists a subsequence $\rho_{1}, \rho_{2}$, $\cdots, \rho_{n}, \cdots, \rho_{n} \rightarrow \infty$, so that $J\left(\rho_{n}\right)=\int_{0}^{\pi}\left|f_{\rho_{n}}(\theta)\right|^{p}(\sin \theta)^{2 \lambda} d \theta \rightarrow 0$.

Let now $h_{\rho}(x, y)$ be the Poisson integral (for the disc $x^{2}+y^{2} \leqq \rho^{2}$ ) of $f_{\rho}(\theta)$ in the sense of $\S \S 2-4$. That is,

$$
h_{\rho}(x, y)=\int_{0}^{\pi} P(r / \rho, \theta, \phi) f_{\rho}(\phi)(\sin \phi)^{2 \lambda} d \phi \quad(x=r \cos \theta, y=r \sin \theta) .
$$

Then we observe that $h_{\rho}(x, y)$ is continuous in $x^{2}+y^{2} \leqq \rho^{2}$, even in $y$, and odd in $x$, the latter because of the "oddness" of $f$. Thus $h_{\rho}(0, y)=0$. Moreover $h_{\rho}(x, y)$ satisfies the equation (16.2) and majorizes $u(x, y)$ on the half-circle $x^{2}+y^{2}=\rho^{2}, x \geqq 0$, and also when $x=0$. Thus by the maximum principle (Theorem 1)

$$
u(x, y) \leqq h_{\rho}(x, y) \quad \text { whenever } x^{2}+y^{2} \leqq \rho^{2}, \quad x \geqq 0 .
$$

We now restrict the $\rho$ 's to our sequence $\rho_{n} \rightarrow \infty$. Since $\int_{0}^{\pi}\left|f_{\rho_{n}}(\theta)\right|^{\rho}(\sin \theta)^{2 \lambda} d \theta \rightarrow 0$, it follows easily that for each fixed $(x, y), h_{\rho_{n}}(x, y) \rightarrow 0$. This proves the lemma. The corollary is an immediate consequence.

(b) The analogue of Lemma 1 are the estimates

$$
\begin{aligned}
& P(x, t, z) \leqq \frac{c x(y z)^{-\lambda}}{x^{2}+(y-z)^{2}}, \\
& P(x, y, z) \leqq \frac{c x}{\left(x^{2}+(y-z)^{2}\right)^{\lambda+1}},
\end{aligned}
$$

where $P$ is given by $\left(16.1^{\prime}\right)$ (or $(16.4)$ ).

(c) For $f \in L^{p}\left(y^{2 \lambda} d y\right)$, the maximal function $f^{*}$ is defined by

$$
f^{*}(y)=\sup _{0 \leqq y+h} \frac{\int_{y}^{y+h}|f(z)| z^{2 \lambda} d z}{\int_{y}^{y+h} z^{2 \lambda} d z} .
$$

The obvious form of Lemma 2 holds for this variant of $f^{*}$, and we also have $\sup _{x}|u(x, y)| \leqq c f^{*}(y)$, where $u$ is given by (16.1). 
(d) Theorems 2, 3, 3', and Corollary 2 (in \$6) have their obvious analogues in this context. As an example of this we state

ANAlogue OF THEOREM 3. Let $u(x, y)$ satisfy (16.2) in the quarter-plane $x>0, y>0$; then $u(x, y)$ is the Poisson integral (16.1) of an $f \in L^{p}\left(y^{2 \lambda^{*}} d y\right)$, $1<p<\infty$, if and only if $u(x, y)$ is regular and even in $y$ in the half-plane $x>0$, and $\sup _{x<0} \int_{0}^{\infty}|u(x, y)|^{p} y^{2 \lambda} d y<\infty$.

The proof of this theorem is almost identical with that of Theorem 3, except that we need to appeal to the corollary of Lemma 11 of this section.

(e) For the conjugate kernel $Q(x, y, z)$ (given by (16.5)) we have the following estimates:

$$
\begin{aligned}
& Q(x, y, z)=O\left(y^{-2 \lambda-1}\right), \quad \text { if } y \leqq z / 2, \\
&=O\left(z^{-2 \lambda-1}\right), \quad \text { if } z \leqq y / 2, \\
& Q(x, y, z)=c_{\lambda}(y z)^{-\lambda} \cdot \frac{y-z}{x^{2}+(y-z)^{2}}+O\left(y^{-2 \lambda-1}\left(1+\log ^{+}\left(\frac{y z}{y-z}\right)\right)\right), \\
& \text { if } y / 2 \leqq z \leqq 2 y .
\end{aligned}
$$

(f) The analogue of Theorem 4 (part (a)) is the statement

$$
\sup _{0<x}\left(\int_{0}^{\infty}|V(x, y)|^{p} y^{2 \lambda} d y\right)^{1 / p} \leqq A_{p} \int_{0}^{\infty}\left|f(y)^{p}\right| y^{2 \lambda} d y^{1 / p}, 1<p<\infty,
$$

with $V$ defined by $V(x, y)=\int_{0}^{\infty} Q(x, y, z) f(z) z^{2 \lambda} d z$.

(g) Going back to formula (16.5) for $Q$ and letting $x \rightarrow 0$, we can reinterpret (f) above as follows:

Suppose $F$ is the Fourier-Bessel transform of $f$, i.e.,

$$
f(y)=\int_{0}^{\infty} V_{\lambda-1 / 2}(y t) F(t) t^{2 \lambda} d t .
$$

Then

$$
\lim _{x \rightarrow 0} V(x, y)=\tilde{f}(y)=-x \int_{0}^{\infty} V_{\lambda+1 / 2}(y t) F(t) t^{2 \lambda+1} d t .
$$

The mapping $f \rightarrow \tilde{f}$ defined thus (initially only for $L^{2}\left(y^{2 \lambda} d y\right)$ ) is the analogue of the Hilbert transform in this case. It is clear from the above that

$$
\int_{0}^{\infty}|f(y)|^{2} y^{2 \lambda} d y=\int_{0}^{\infty}|f(y)|^{2} y^{2 \lambda} d y .
$$

The result in (e) shows that the mapping $f \rightarrow \tilde{f}$ extends to $L^{p}\left(y^{2 \lambda} d y\right), 1<p<\infty$, and is boundded there.

(h) The results for $H^{p}$-spaces (as in $\S \S 9$ and 10) can be developed in this 
case also. The analogue of the harmonic majorization lemma (Theorem 5) requires now the additional Lemma 11 of this section for its proof. Otherwise the arguments are essentially identical. Theorem 6 has its analogue which may be stated as follows.

AnAlogue of Theorem 6. Suppose $F(z)=U(x, y)+i V(x, y)$ satisfies (16.6') with $U$ even and $V$ odd in $y$. Suppose also that for $p \geqq 2 \lambda /(2 \lambda+1)$, $\sup _{x>0} \int_{0}^{\infty}|F(x+i y)|^{p} y^{2 \lambda} d y<\infty$. Then (a) $\lim _{x \rightarrow 0} F(x+i y)=F(i y)$ exists for almost every $y$, even in the nontangential sense. (b) If $\mid F(i y)=0$ on a set of positive measure, then $F(z) \equiv 0$. (c) If $p>2 \lambda /(2 \lambda+1)$, then $\lim _{x \rightarrow 0} \int_{0}^{\infty}|F(x+i y)-F(i y)|^{p} y^{2 \lambda} d y=0$.

This theorem (in particular (c) when $p=1$ ) has then the following

Corollary. Suppose we have a pair of measures $d \mu_{1}, d \mu_{2}$, so that $\int_{0}^{\infty} y^{2 \lambda}\left|d \mu_{i}(y)\right|<\infty$. If $\int_{0}^{\infty} V_{\lambda-1 / 2}(x y) y^{2 \lambda} d \mu_{1}(y)=-x \int_{0}^{\infty} V_{\lambda+1 / 2}(x y) y^{2 \lambda+1} d \mu_{2}(y)$, then both $d \mu_{i}$ are absolutely continuous with respect to Lebesgue measure.

This, of course, is another extension of the classical F. and M. Riesz theorem on absolute continuity of analytic measures.

(i) Coming now to the results of Chapter II, and in particular those of $\S 14$, we shall limit ourselves to the two main statements in the present version.

(1) Suppose we consider the multiplier transformation $T$, intially defined on $L^{2}\left(y^{2 \lambda} d y\right)$ by

where

$$
(T f)(y)=\int_{0}^{\infty} F(t) \mu(t) V_{\lambda-1 / 2}(y t) t^{2 \lambda} d t,
$$

$$
F(t)=\int_{0}^{\infty} V_{\lambda-1 / 2}(y t) f(y)^{2 \lambda} d y
$$

with the multiplier $\mu(t)$ of bounded variation in every finite closed subinterval of $(0, \infty)$ and satisfying $|\mu(s)| \leqq M$ and $\int_{0}^{s} t|d \mu(t)| \leqq M s, 0<s<\infty$. Then the transformation $f \rightarrow T f$ has a unique bounded extension from $L^{p}\left(y^{2 \lambda} d y\right)$ to itself, with $(2 \lambda+1) /(\lambda+1)<p<(2 \lambda+1) / \lambda(11)$.

(2) Suppose we define the partial integrals $S_{a}(f)$, by

$$
S_{a}(f)=\int_{0}^{a} F(t) V_{\lambda-1 / 2}(y t) t^{2 \lambda} d t .
$$

If $f \in L^{p}\left(y^{2 \lambda} d y\right)$, then $S_{a_{k}}(f) \rightarrow f$ almost everywhere and dominately (in $L^{p}$ ), for $(2 \lambda+1) /(\lambda+1)<p<(2 \lambda+1) / \lambda$, whenever $a_{k}$ is lacunary in the sense that $\liminf _{k \rightarrow \infty}\left(a_{k+1} / a_{k}\right)>1$.

(11) The result is extendable to other $L^{p}$-spaces involving weight functions (see the remarks in \$17). In this way one obtains anew the "Main Theorem" of [6]. 
(j) Finally we come to the topic of (fractional) potentials dealt with in $\$ 15$. Let us define the operators $I_{\alpha}$ by

with

$$
I_{\alpha}(f)=\int_{0}^{\infty} F(t) V_{\lambda-1 / 2}(t y) t^{-\alpha} t^{2 \lambda} d t
$$

$$
F(t)=\int_{0}^{\infty} V_{\lambda-1 / 2}(t y) f(y) y^{2 \lambda} d y,
$$

at least when $f$ is continuous with compact support and $0<\alpha<2 \lambda+1$. Now it is known that $\lim _{\varepsilon \rightarrow 0} \int_{0}^{\infty} e^{-\varepsilon t} V_{\lambda-1 / 2}(t y) t^{-\alpha} t^{2 \lambda} d t=c_{\alpha, \lambda} y^{-2 \lambda-1+\alpha}$. (See [21, p. 391].)

Then if terms of the known convolution structure for the Fourier-Bessel transforms (see Bochner [4]) we can write

$$
I_{\alpha}(f)=f * K_{\alpha}, \quad \text { where } K_{\alpha}(y)=c_{\alpha, \lambda}^{\prime} y^{-2 \lambda-1+\alpha} .
$$

The general Theorem 13 of $\$ 15$ then applies and shows that $\left\|I_{\alpha}(f)\right\|_{r} \leqq A_{r, p}\|f\|_{p}$, with $1<p<r<\infty$ and $1 / r=1 / p-\alpha /(2 \lambda+1)$.

17. Remarks on weighted inequalities. In some problems it is of interest to extend the usual statements of $L^{p}$ boundedness by modifying the measures with suitable weight factors $\left({ }^{12}\right)$.

Thus in the ultraspherical case one would look for inequalities of the form

$$
\left\|T(f)(\theta)(\sin \theta)^{\alpha}\right\|_{p} \leqq A_{p, \alpha}\left\|f(\theta)(\sin \theta)^{\alpha}\right\|_{p}
$$

with $\|f\|=\left(\int_{0}^{\pi}|f(\theta)|^{p}(\sin \theta)^{2 \lambda} d \theta\right)^{1 / p}$.

Similarly in the Fourier-Bessel transform case one would have

$$
\left\|T(f)(y) y^{\alpha}\right\|_{p} \leqq A_{p, \alpha}\left\|f(y) y^{\alpha}\right\|_{p}
$$

with $\|f\|_{p}=\left(\int_{0}^{\infty}|f(y)|^{p} y^{2 \lambda} d y\right)^{1 / p}$.

The validity of such inequalities falls in two ranges. The broader range

$$
\frac{-(2 \lambda+1)}{p}<\alpha<\frac{2 \lambda+1}{p^{\prime}}, \quad \frac{1}{p}+\frac{1}{p^{\prime}}=1
$$

and the narrower range

$$
\frac{-(2 \lambda+1)}{p}+\lambda<\alpha<\frac{2 \lambda+1}{p^{\prime}}-\lambda \quad(\text { recall } \lambda \geqq 0) .
$$

Thus in the case of ultraspherical expansions Theorems 2((b)-(d)), 3, 4(a), Corollaries 1 and 3 and Theorem 8 are all extensible in this sense, with the $L^{p}$-norm taken in the broader sense $\left(R_{1}\right)$. This extension can be carried out without any difficulty, and goes back to the fact that those theorems in question are based

(12) See in particular [1], [6], and [8], where further references can be found. 
on estimates for the Poisson and conjugate kernels which allow such variants. This situation is also typified by similar inequalities in Euclidean $n$-space, (where $n=2 \lambda+1)$, treated in [15].

However, results which essentially go back to partial sums, Theorems 7,9 , 10 and 11 , are valid only in the narrower range $\left(R_{2}\right)$. This situation is already typified, to some extent, by some of the results in [13] and [6].

A similar situation in terms of the inequalities (17.2) holds throughout for the Fourier-Bessel transforms considered in $\$ 16$.

18. Concluding remarks: Fourier-Bessel series. The general setting which we used above seems applicable to a variety of other classical expansions. We shall illustrate this by describing the formal setting in the case of Fourier-Bessel series. While we have not pursued this matter any further, it seems highly likely that the analogy with the above can be pushed quite far.

From our point of view the Fourier-Bessel series (also referred to as Dini series) arise by studying functions $u\left(x, y_{1}, \cdots, y_{n}\right)$ harmonic in the cylinder $x>0, y_{1}^{2}+y_{2}^{2}+\cdots+y_{n}^{2}<1$, of the $(n+1)$-dimensional Euclidean space; these functions are given by their boundary values at $x=0, u\left(0, y_{1}, y_{2}, \cdots, y_{n}\right)=f\left(y_{1}, \cdots, y_{n}\right)$, and have vanishing normal derivative along the rest of the boundary( $\left.{ }^{13}\right)$. By restricting consideration to radial functions $f$, we obtain Fourier-Bessel series expansions of order $(n-2) / 2$; and then by analogy we pass to Fourier-Bessel series of real order greater than $-1 / 2$.

Thus if $u\left(x, y_{1}, \cdots, y_{n}\right)=u(x, y), \quad\left(y=\left(y_{1}^{2}+\cdots+y_{n}^{2}\right)^{1 / 2}\right)$, is harmonic in $x>0,0 \leqq y<1$, and satisfies $u(0, y)=f(y)$, with $u_{y}(x, 1)=0$, then

$$
u(x, y)=\sum_{m=0}^{\infty} a_{m} e^{-\gamma_{m} x} V_{\lambda-1 / 2}\left(\gamma_{m} y\right) \quad(2 \lambda=n-1),
$$

where $V_{\lambda}(t)=t^{-\lambda} T_{\lambda}(t)$, and $\gamma_{0}=0, \gamma_{1}, \gamma_{2}, \cdots, \gamma_{m} \cdots$ are the successive zeros of $T_{\lambda-1 / 2}^{\prime}(t)$. We may now drop the assumption that $2 \lambda$ is integral, and assume only $\lambda \geqq 0$.

The natural $L^{p}$-norms in this case are taken (as before) with the measure $y^{2 \lambda} d y$; thus $\|f\|_{p}=\left(\int_{0}^{1}|f(y)|^{p} y^{2 \lambda} d y\right)^{1 / p}$. For $p=2$ we know that the collection $\left\{V_{\lambda-1 / 2}\left(\gamma_{m} y\right)\right\}$ is mutually orthogonal and as a matter of fact complete. (See Watson [21, Chapter 18].)

We should observe that from (18.1) we obtain the expansion

$$
f(y) \sim \sum_{m=0}^{\infty} a_{m} V_{\lambda-1 / 2}\left(\gamma_{m} y\right)
$$

$u(x, y)$ given by (18.1) satisfies the singular Laplace equation (16.2) and thus we should expect its conjugate $V$ to be given by (16.6). From this it follows that

(13) This boundary condition is typical of a more general class that can be treated similarly. 


$$
V(x, y)=-y \sum_{m=1}^{\infty} a_{m} e^{-\gamma_{m} x} \gamma_{m} V_{\lambda+1 / 2}\left(\gamma_{m} y\right)
$$

If we now let $x \rightarrow 0$, we obtain the function $\tilde{f}(y)$ conjugate to $f(y)$, given by

$$
\tilde{f}(y) \sim-y \sum_{m=1}^{\infty} a_{m} \gamma_{m} V_{\lambda+1 / 2}\left(\gamma_{m} y\right) .
$$

(Compare this notion of conjugacy with (2.4) for ultraspherical series and (16.7)-(16.8) for Fourier-Bessel transforms.)

We state now two results which can be expected to hold in view of the close analogy with the above, and which would be typical. The first would be another extension of the M. Riesz conjugate function inequality, and would take the form that the mapping $f(y) \rightarrow \tilde{f}(y)$ (given by (18.4) is bounded in the $L^{p}$-norm (as defined above) with $1<p<\infty$.

Secondly, one could reasonably expect an analogue of the multiplier theorem of $\S 14$. It would state:

Let the transformation $f \rightarrow T f$ be given by

$$
T f \sim \sum_{m=0}^{\infty} a_{m} \mu_{m} V_{\lambda-1 / 2}\left(\gamma_{m} y\right)
$$

with f given by (18.2) and $\mu_{m}$ satisfying:(i) $\left|\mu_{k}\right| \leqq M$,(ii) $\sum_{m=1}^{k} m\left|\mu_{m}-\mu_{m-1}\right| \leqq M k$, all $k$. Then the transformation is bounded in the $L^{p}$-norm, with $(2 \lambda+1) /(\lambda+1)$ $<p<(2 \lambda+1) / \lambda$.

\section{REFERENCES}

1. R. Askey and I. I. Hirschman, Weighted quadratic norms and ultraspherical polynomials. I, Trans. Amer. Math. Soc. 91 (1959), 294-313.

2. L. Bers, Function-theoretical properties of solutions of partial differential equations of elliptic type, Contributions to the theory of partial differential equations, pp. 69-94, Annals of Mathematics Studies No. 33, Princeton Univ. Press, Princeton, N. J., 1954.

3. L. Bers and A. Gelbart, On a class of differential equations in mechanics of continua, Quart. Appl. Math. 1 (1943), 168-188.

4. S. Bochner, Sturm-Liouville and heat equations whose eigenfunctionsareultraspherical polynomials or associated Bessel functions, Proceedings of the conference on differential equations, pp. 23-48, Univ. of Maryland, College Park, Md., 1955.

5. S. Bochner and K.Chandrasekharan, Fourier transforms, Annals of Mathematics Studies No. 19, Princeton Univ. Press, Princeton, N. J., 1949.

6. D. L. Guy, Hankel multiplier transformations and weighted p-norms, Trans. Amer. Math. Soc. 95 (1960), 137-189.

7. C. S. Herz, On the mean inversion of Fourier and Hankel transforms, Proc. Nat. Acad. Sci. U.S.A. 40 (1954), 996-999.

8. I. I. Hirschman, The decomposition of Walsh and Fourier series, Mem. Amer. Math. Soc. No. 15 (1955), 65 pp.

9. E. Hopf, Elementare Bemerkungen über die Lösungen partieller Differentialgleichungen von elliptischen Typus, S.-B. Berlin Math. Ges. (1927), 147-152. 
10. J. Horvath, Sur les fonctions conjuguées à plusieurs variables, Nederl. Akad. Wetensch. Proc. Ser. A. 16 (1953), 17-29.

11. A. Huber, On the uniqueness of generalized axially symmetric potentials, Ann. of Math. (2) 60 (1954), 351-358.

12. R. O'Neil, Convolution operators and $L(p, q)$ spaces, Duke Math. J. 30 (1963), 129-142.

13. H. Pollard, The mean convergence of orthogonal series of polynomials, Proc. Nat. Acad Sci. U.S.A. 32 (1946), 8-10.

$14 \longrightarrow$, The mean convergence of orthogonal series. II, Trans. Amer. Math. Soc. 63 1948), 355-367.

15. E. M. Stein, Note on singular integrals, Proc. Amer. Math. Soc. 8 (1957), 250-254.

16 - Conjugate harmonic functions in several variables, pp. 414-420, Internat. Congress of Mathematicians, Stockholm, 1962.

17. E. M. Stein and G. Weiss, On the theory of harmonic functions of several variables. I, Acta Math. 106 (1961), 137-174.

18. G. Szegö, Orthogonal polynomials, Amer. Math. Soc. Colloq. Publ. Vol. 23, Amer. Math. Soc., Providence, R. I., 1939.

19. E. C. Titchmarsh, Introduction to the theory of Fourier integrals, Clarendon Press, Oxford, 1937.

20. I. N. Vekua, Generalized analytic functions, Pergamon Press, London, 1962(translated from the Russian edition, Fizmatgiz, Moscow, 1959).

21. G. N. Watson, Theory of Bessel functions, Cambridge Univ. Press, Cambridge, 1922.

22. - - Notes on generating functions of polynomials. III. Polynomials of Legendre and Gegenbauer, J. London Math. Soc. 8 (1933), 289-292.

23. A. Weinstein, Discontinuous integrals and generalized potential theory, Trans. Amer. Math. Soc. 63 (1948), 342-354.

24. ——, Generalized axially symmetric potential theory, Bull. Amer. Math. Soc. 59 (1953), 20-38.

25. G. M. Wing, The mean convergence of orthogonal series, Amer. J. Math. 72 (1950), $792-808$.

26. A. Zygmund, Trigonometric series, 2nd. ed., Vols. I, II, Cambridge Univ. Press, New York, 1959.

Rutgers, The State University,

NEW BRUNSWICK, NEW JERSEY

UNiversity of Chicago,

Chicago, Illinois

INSTITUTE FOR ADVANCED STUDY, Princeton, NeW Jersey 